\title{
$\mathrm{N} 7229587$
}

$$
\begin{aligned}
& \text { CASE FILE } \\
& \text { COPX }
\end{aligned}
$$




\section{FRACTURE BEHAVIOR IN NYLON 6 FIBERS}

$$
\text { by }
$$

Ben Ashton Lloyd

A dissertation submitted to the faculty of the University of Utah in partial fulfillment of the requirements

for the degree of

$$
\text { Doctor of Philosophy }
$$

Department of Mechanical Engineering

University of Utah

June 1972 
Page intentionally left blank 


\section{ACKNOWLEDGMENTS}

The author wishes to recognize and express gratitude to all who have made this dissertation possible.

First to Dr. K. L. DeVries, advisor, counselor, and friend whose optimistic support and suggestions made it possible to continue this study even in times of discouragement. Extreme gratitude is expressed to Dr. DeVries for his guidance and the many basic ideas which contributed so much to this research. The many hours spent with him has built a friendship which will be cherished always.

To my friends and office roommates, Joon Bu Park, Keng-Kie Liem, and Bruce Moore, who not only have contributed their knowledge but more importantly their support and encouragement, may success and happiness always touch their lives.

To Dr. M. L. Williams for his support as principal investigator and co-director with Dr. K. L. DeVries of this research; Dr. W. 0 . Statton and Dr. D. K. Roylance for their many suggestions and indepth discussions into the mysteries of polymers; Craig Hansen, George Nichols, and Stephan Nichols for their help in reducing the data and preparing the drawings (without their efforts this study would still be in process); and also to Ruth Sadler, Vicki Nielsen, and Sharon Lambson who have spent so many hours typing, pasting, and correcting this manuscript, I express my deepest gratitude.

Grateful acknowledgment is given to the National Science Foundation for their portion of the financial support of this work. Also 
use was made of facilities purchased under a National Aeronautics and Space Administration grant. Thanks is also given to the National Defense Education Act for the supporting fellowship used to complete the last year of this study.

Last, but most important, grateful appreciation and love is expressed to my lovely wife and four boys who have so willingly sacrificed all that was necessary to complete this work. Without their encouragement and support this achievement would not have been possible. 
TABLE OF CONTENTS

Acknowl edgments

List of Figures -

List of Tables and Diagrams

Abstract -

1.0 INTRODUCTION -1

2.0 BACKGROUND -

2.1 General -....... 5

2.2 Polymer Morphology -....... 8

2.3 Failure Criteria - 12

3.0 EPR FOUNDATIONS -

4.0 EXPERIMENTAL PROCEDURE -

4.1 Experimental Equipment -............................... 22

4.2 Sample Material, Preparation, and Testing -...--.-- 25

5.0 THEORETICAL AND EXPERIMENTAL RESULTS $\ldots \ldots$

5.1 Introduction -..........- 34

5.2 Mathematical Model -

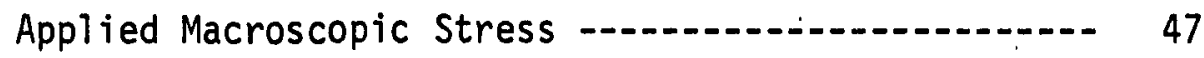

5.3 Model Parameters and Room Temperature Tests -......- 57

The Distribution Parameter "S" -..................... 57

The Chain Ratio Parameter "RC". ................... 71

The Chain Length Parameter "RL" -......... 75

The Modulus of Elasticity of a Single Nylon 6

Polymer Chain "Eb"

The Collision Parameter "Wo" -.................... 78

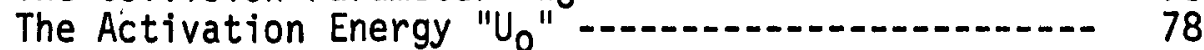

The Activation Volume " $\gamma^{R}$........................ 81

Discussion -...- 92

5.4 Other Temperatures and Materials ....................... 109 
6.0 SUMMARY AND CONCLUSIONS -

6.1 Research Objectives and Approach -................. 136

6.2 Limitations of the Study -.................- 137

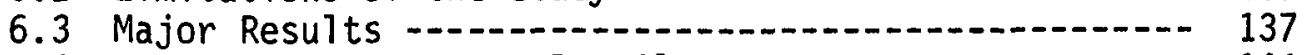

6.4 Summary of Important Details ...................... 141

6.5 Application -...- 148

REFERENCES -

VITA - 
United States annual production of major metals and polymers

Typical line drawings of the fringed micelle model (12)

Microfibril model of fiber structure with folded chain blocks and tie molecules (13)

Paracrystalline layer lattice showing chain back-foldings in the amorphouscrystalline transition regions (14)

Shish-kebab formation caused by stress crystallization in polyethylene (22)

Structure of nylon 6 fibers and

alterations due to slack and tension annealing proposed by Park (23)

Zeeman splitting for a free electron

EPR spectra of nylon 6 at room temperature

EPR spectrometer with servo-controller on the left and load frame and spectrometer unit on the right configuration and dimensions nyion 6 \#1 ( $x$ denotes strands to be cut after bonding) to be cut after bonding) 

stepwise loading of sample at room temperature

Histograms of nylon 6 samples from step strain data at room temperature

Probability plot of nylon 6 histogram data

Idealized model of fiber structure showing microscopic block regions cal flaw" region of idealized fiber microstructure step-strain data at various temperatures step-strain data at various temperatures from step-strain data at room temperature strain data at room temperature effective tie-chain distribution with temperature

Schematic representation of irreversible type changes created in the tie-chain distribution under slack and tension annealing

Relationship between temperature and effective distribution for various sample materials of nylon 6 

the steady state chain scission rate, $\dot{C}_{s s}$, and the applied macroscopic stress for various loadings

Constant strain rate tests for nylon 6 \#1 at room temperature for nylon 6 \#1 at room temperature for nylon 6 \#1 at room temperature

Cyclic stress fatigue $(0.05 \mathrm{cycle} / \mathrm{sec})$ test for nylon 6 \#1 at room temperature

Creep test with stress
to theoretical model

Constant strain rate test with different theoretical parameters Effect of apparent distribution on maximum
strength for nylon $6 \# 2$ at several temperatures 

theoretical predictions for nylon $6 \# 1$ at $-25^{\circ} \mathrm{C}$, room temperature, and $+50^{\circ} \mathrm{C}$ results with respect to two types of distribution changes

Effect of temperature on accumulative chain scissions at fracture and at maximum stress point for nylon $6 \# 1$ cal predictions for nylon $6 \# 2$ at $-25^{\circ} \mathrm{C}$, room temperature, and $+500 \mathrm{C}$ scissions at fracture and at maximum stress point for nylon $6 \# 2$ distribution for one set of specific input parameters

Nylon 66 constant strain rate room temperature tests 


\section{LIST OF TABLES AND DIAGRAMS}

$\underline{T a b l e s}$

Table

Title

Page

1

EPR spectrometer performance specifica-

24 tions

2

Linear relationships observed between the steady state chain scission rate, $\hat{C}_{s s}$, and the applied macroscopic stress for various loadings

3

" $\gamma$ " values for nylon 6 obtained from Figure 32

4

Model parameters

108 and 143

\section{Flow Diagrams}

Flow Diagram

Titie

Page

Flow Diagram 1

Distribution Computer Program

$55 \mathrm{a}$

Flow Diagram 2

Stress-Strain Bonds Computer Program

$55 b, c$ 
ABSTRACT

Electron Paramagnetic Resonance (EPR) techniques are used to determine the number of free radicals produced during deformation leading to fracture of nylon 6 fibers. A reaction rate molecular model is proposed to explain some of the deformation and bond rupture behavior leading to fracture. High-strength polymer fibers are assumed to consist of a sandwich structure of disordered and ordered regions along the fiber axis. In the disordered or "critical flaw" regions, tie chains connecting the ordered or "crystalline block" regions are assumed to have a statistical distribution in length. These chains are, therefore, subjected to different stresses. The effective length distribution was determined by EPR. The probability of bond rupture was assumed to be controlled by reaction-rate theory with a stress-aided activation energy and behavior of various loadings determined by numerical techniques. The model is successfully correlated with experimental stress, strain, and bond rupture results for creep, constant rate loadings, cyclic stress, stress relaxation and step strain tests at room temperature. Successfur correlations are a) so made for constant strain rate tests at $-25^{\circ} \mathrm{C}$ and $+50^{\circ} \mathrm{C}$. The effective length distribution is found to be temperature dependent and directly related to the strength and other fiber properties. Preliminary results are presented indicating feasible extension of the model to other high strength materials such as nylon 66 . 


\subsection{INTRODUCTION}

The prediction and modification of strength on useful service life in polymeric materials is becoming a progressively more important scientific and technological problem with the very rapid increase in the amount and type of polymer usage. Figure 1 shows that in 1930 the United States annual production of polymer materials was less than any of the major metals, i.e. steel, aluminum and copper. In 1962, on a volume basis, polymer production exceeded all other other combined metal production except steel, and it was predicted even to exceed steel in the next few years (1). In an article published in 1969, it was noted that synthetic fibers have increased 5,300 per cent in the past 18 years and that man-made fibers accounted for nearly 40 per cent of the total fiber production of the world (2).

With such growth has come a tremendous need for understanding the strength and failure phenomena of these materials. The word "strength" is necessarily related to the term "failure" since they represent the positive and negative aspects of the same phenomena the load-bearing capacity of a material.

One can approach fracture phenomenon from two distinctly different points of view, either the submicroscopic (atomic) approach or the macroscopic standpoint. In the atomic approach, one attempts to relate external loadings to molecular forces which in turn result in chemical bond rupture. The accumulation of these broken bonds then leads to the final gross fracture of the specimen or engineering element. 


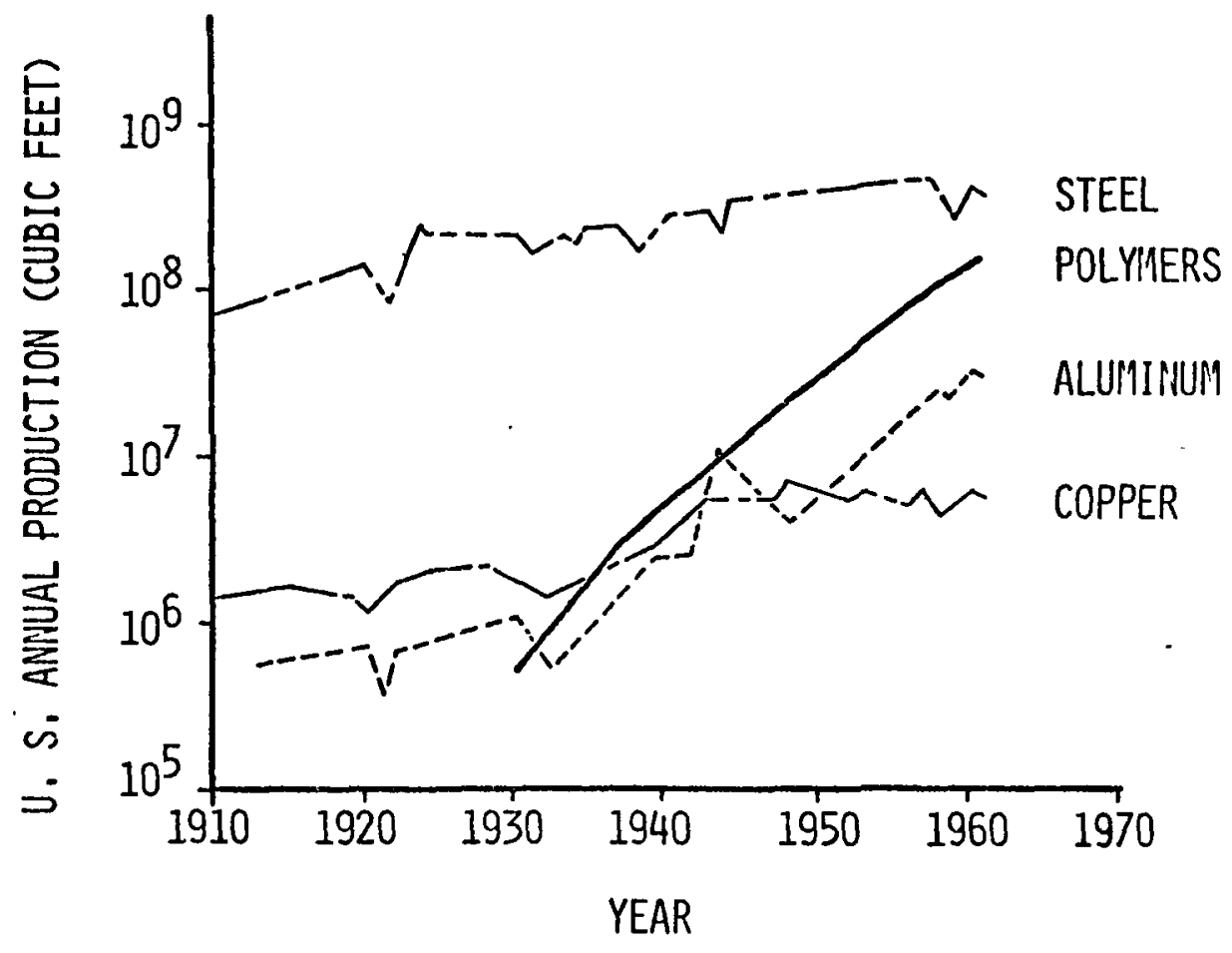

FIGURE 1. United States annual production of major metals and polymers 
On the other hand, in the past, many criteria or theories of failure have treated materials as continua. In these criteria, the material is generally treated as a continuum or at least a quasicontinuum with mathematically introduced flaws or holes. The emphasis is then placed on devising some functional relationships such as an energy balance or statistical analys is of crack nucleation or growth dealing with the local stress and/or strain in the material. To date certainly, this latter approach has received the most attention in engineering analysis. It allows the prediction of failure for specific loadings and sometimes provides the engineer with a satisfactory design tool. However, since basically materials are not continua, a thorough understanding of fracture must include an understanding of occurrences of an atomic or molecular scale. Such an understanding is important to the materials engineer and scientist from a very practical standpoint. Only through such an understanding is it possible to relate the chemical and structural properties on an atomic or molecular scale to the macroscopic behavior. With such relationships, the materials engineer and scientist can then gain greater insight into expected behavior under various loading conditions, effects of polymerization, annealing, drawing or other special processes on this behavior and make quantitatively meaningful extrapolations for designing and analyzing new materials.

Many researchers have proposed theories and molecular models to explain fracture from a microscopic viewpoint (3-7). However, theoretical predictions of failure on a microscopic scale could not be experimentally verified until 1964 when a Russian group of scientists under the direction of S. N. Zhurkov demonstrated that 
Electron Paramagnetic Resonance (EPR) could be used to measure chain scission caused by mechanical stress in polymers (8). Since that time, a number of researchers as well as ourselves have been conducting fracture studies using EPR techniques with only partial success in establishing a satisfactory failure criterion (9).

The objective of this study was to develop a successful failure criterion for highly oriented polymer fibers which would relate microscopic behavior to the macroscopic properties observed during the failure process. Such a failure criterion was established for nylon 6 and not only allows prediction of macroscopic behavior under various loading and temperature conditions, but it also gives insight into a systematic method by which the micro-structure may be changed to vary the properties of the polymer fiber. 


\subsection{BACKGROUND}

\subsection{GENERAL}

Polymers are certainly not a new material as many such substances exist in nature, i.e. rubber, cellulose, collagen, etc; however, man-made polymers or plastics have become available only within the last 40 years.

Basically, polymers are composed of very long molecules entwined with each other in some ordered or disordered arrangement. Each of these long molecules is built up from a basic repeat or monomer unit. Some repeat units for various polymers are shown in Figure 2 (10). As repeat units are added together, the material has properties of an oil, wax or powder until the chains reach lengths greater than $\sim 1000 A^{0}$, after which the material begins to exhibit the toughness, strength and viscoelastic properties that are characteristic of polymers. The Number Average Molecular Weight (i. e. the weight of the average chain in the polymer) should always exceed 10,000 to obtain characteristic polymer properties; however, for short repeat unit polymers, like polyethylene, molecular weights above 100,000 may be necessary to obtain an average chain length greater than $1000 \mathrm{~A}^{0}$.

Polymers are classified into two general catagories; amorphous and semi-crystalline. Amorphous polymers are thought to have no definite form or large scale order whereas semi-crystalline have an ordered pattern to their structure and even regions which may be crystallized. One might visualize an amorphous polymer as much like a randomly oriented mass of spagetti where the spagetti represent the entangled polymer chains. From this analogy, it is easy to see that 


$$
\left[\begin{array}{cccccc}
-N & -\left(\mathrm{CH}_{2}\right)_{5} & -C & -\mathrm{N} & -\left(\mathrm{CH}_{2}\right)_{5} & -\mathrm{C} \\
\mathrm{H} & \mathrm{W} & 1 & - \\
\mathrm{H} & 0 & \mathrm{H} & 0
\end{array}\right] n
$$

Poly-६-caprolactam (Nyion 6)

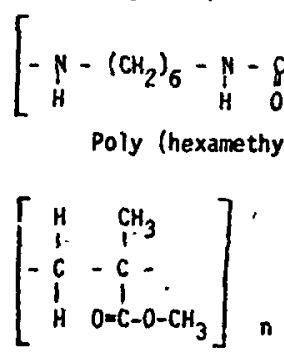

Poly (methyl methacrylate) (PMMA)

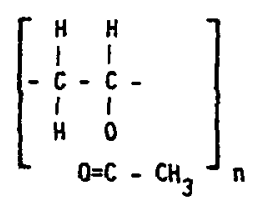

Poly(vinyl acetate)

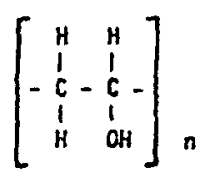

Poly (Viryl alcohol)
$\left[\begin{array}{cc}H & H \\ 1 & 1 \\ -C & -C \\ 1 & 1 \\ H & H\end{array}\right]_{n}$

Polyethylene (PE)

$\left[\begin{array}{cc}H & H \\ 1 & 1 \\ -C & -C \\ 1 & 1 \\ H & 0 \\ H\end{array}\right]$

Polystyrene (PS)

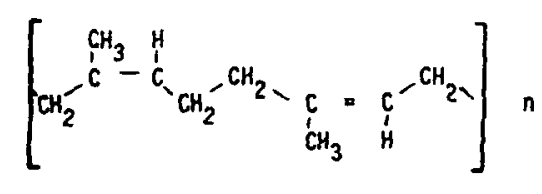

Poly-cis-1,4-isoprene (natural rubber-hevea)

Lexan* $\quad\left[\begin{array}{cc}H & H \\ 1 & 1 \\ -C & -C \\ 1 & 1 \\ H & C l\end{array}\right]_{n}$

Poly (vinyl chloride) (PVC)

Deirin*

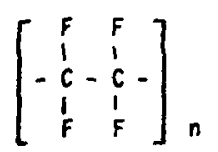

Poly (tetrafluoroethylene) (PTFE) 
failure of a polymer structure may occur in two ways. Under a given load either chains will slip past each other and unwind the structure, or backbone chain scission can occur causing eventual failure. The effect of temperature on such a process is certainly an important factor. If we visualize the structure as in a constant state of internal vibration and motion, then the intensity of this motion will greatly effect macroscopic properties and the mode of failure. This motion can be localized or fairly widespread and depends on the intramolecular chain flexibility, the inter-molecular forces between chains, and the overall configuration of the chain structure, all of which are temperature sensitive.

In some polymers, a glass temperature or glass transition point $\left(T_{g}\right)$ is designated. Below the glass transition temperature, the material acts very much like a glassy, brittle substance, whereas above $T_{g}$ the polymer is more flexible and rubber-like. Generally, only amorphous type polymers have a clearly defined glass transition temperature.

Failure in most polymer materials is generally a combination of both slippage and chain scission phenomena, however, it is thought that some highly ordered semi-crystalline polymers exhibit more backbone chain scission during failure than do amorphous polymers (11). It was this observation that led to the use of Electron Paramagnetic Resonance (EPR) as an instrument for studying failure in highly ordered semi-crystalline polymer materials such as high strength fibers and films (8). The fundamentals of EPR are explained in section 3.0. Basically, it is a spectroscopy method by which backbone chain scission (covalent chemical bond failure) can be detected. 


\subsection{POLYMER MORPHOLOGY}

The morphology of highly-oriented semi-crystalline polymers has been the subject of much past and current research. This research effort is not a morphological study, however, the author has attempted to take basic structural morphological parameters as reported by knowledgeable researchers and construct an idealized physical model relating macro-loadings to this micro-structure in order to predict failure in polymer fibers.

Several models have been proposed to describe the microstructure in highly oriented fibrous polymers. These range from the early fringed micelle structures shown in Figure 3 (12) to more recently proposed folded chain models, represented in Figures 4 and $5(13,14)$. Although models proposed by Hearle (15), Bosley (16), Keller (17), Fischer, et al (18), Peterlin (13), Dismore and Statton (19), Gubanov and Chevychelov (20), Takayanagi, et al (21), and Bonart and Hosemann (14) differ in some very important details, they have a basic common element in their microstructure; that is, a11 include a "sandwich" type structure of alternating ordered or semi-ordered regions and disordered or tie molecule regions. Electron micrograph studies also give supporting evidence for the above concept of rheological block and tie molecule regions. An electron micrograph of stress crystallized polyethylene from the melt is shown in Figure 6 (22). The relationship of this so-called "shish-kebab" structure to drawn fibers is not yet clear, although some aspects of stress crystallization may play a part in melt spun, hot drawn fibers. For the particular melt spun, hot drawn nylon 6 fibers used in this research, a verv excellent morphological study has been recently completed by Joon 

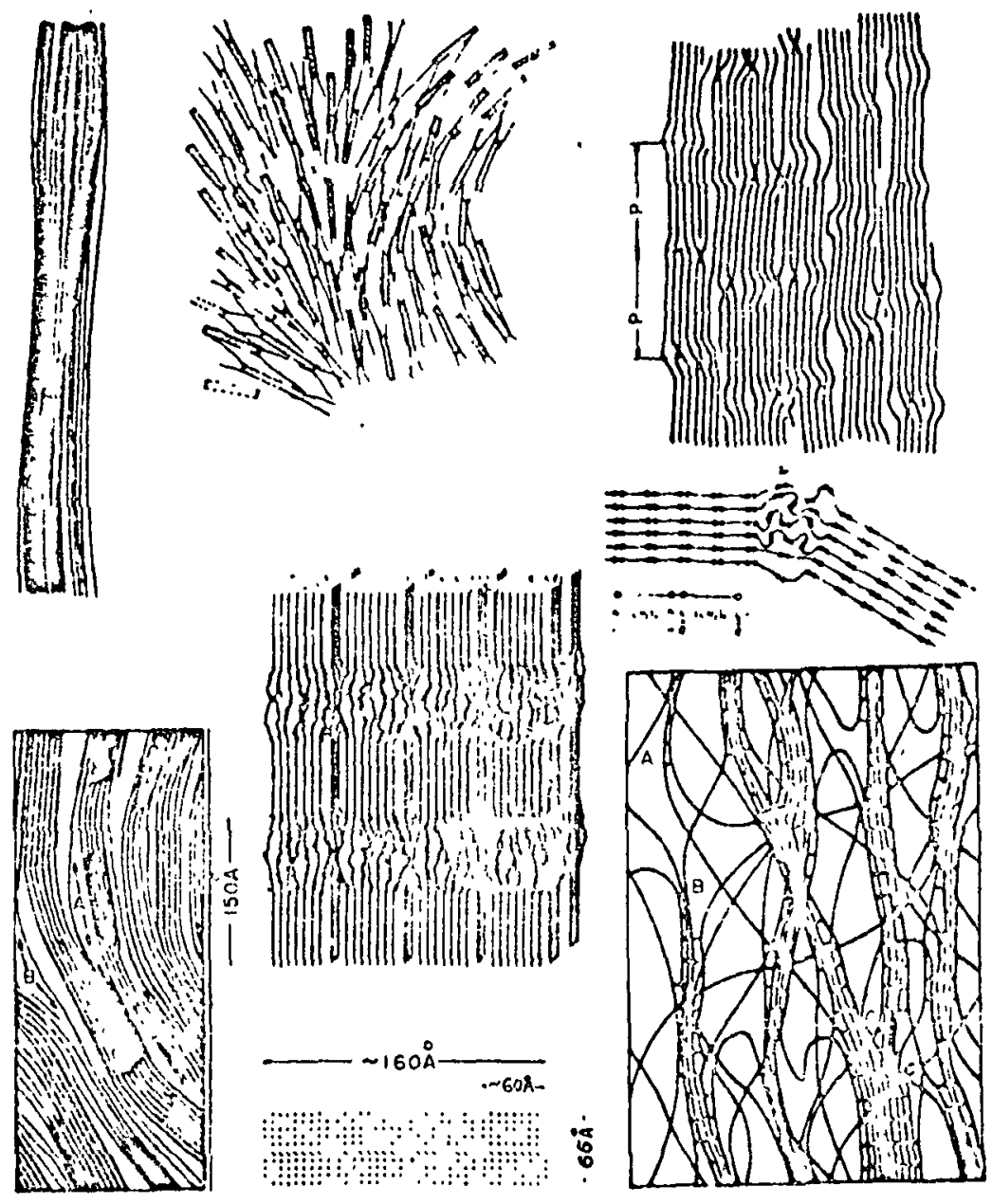

FIGURE 3. Typical line drawings of the fringed micelle model (12). 


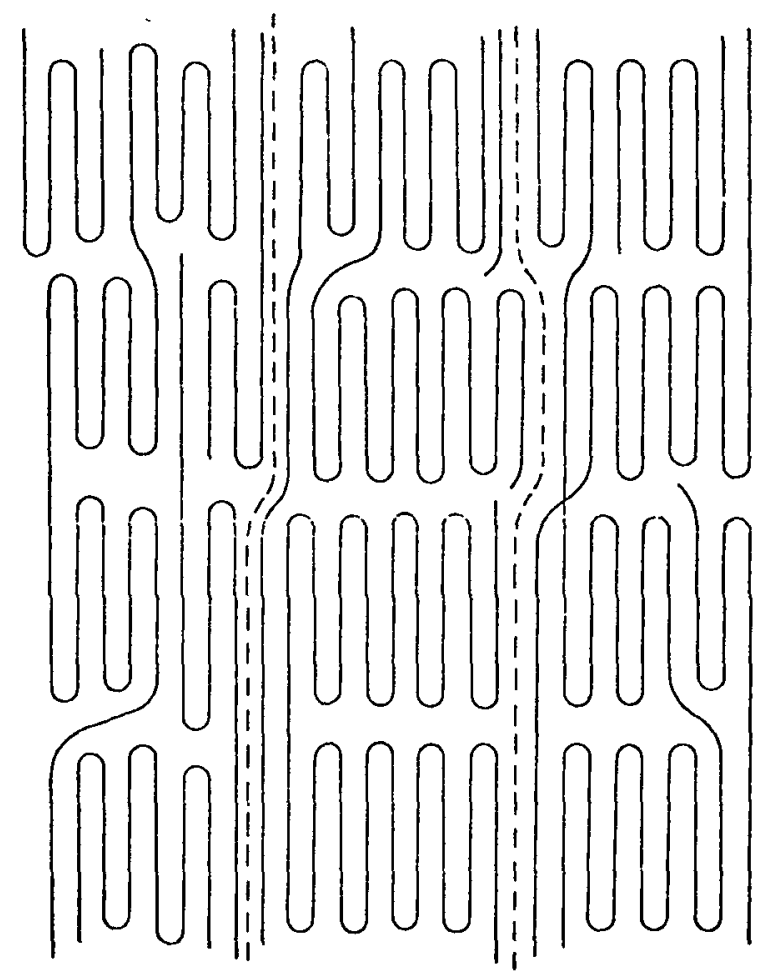

FIGURE 4. Microfibril model of fiber structure with folded chain blocks and tie molecules (13).

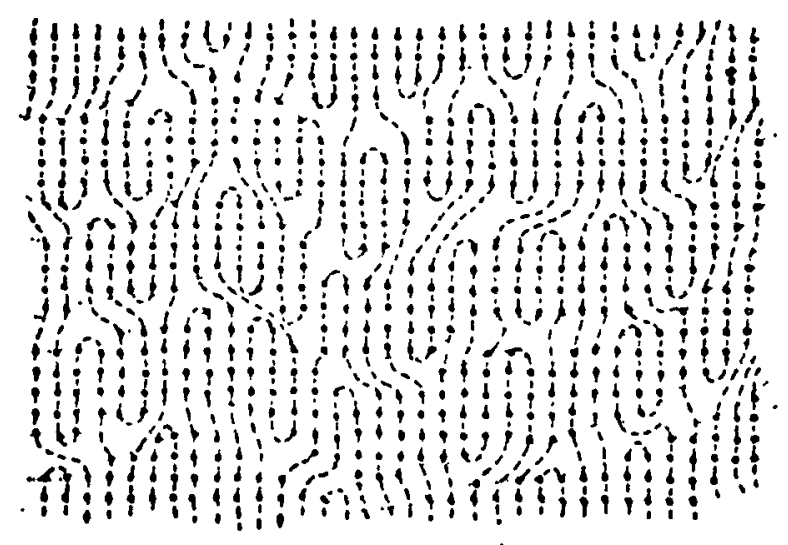

FIGURE 5. Paracrystalline layer lattice showing chain back-foldings in the amorphous-crystalline transition regions (14). 
Page Intentionally Left Blank 
Park (23) under the direction of W. 0 . Statton and K. L. DeVries. From their study, a paraarystalline, folded chain structure is proposed as a representative model of the fiber microstructural (Figure 7).

\subsection{FAILURE CRITERIA}

Although in the most general sense structural failure can be a matter of excessive deformation as well as outright fracture, we shall be concerned here only with the latter case in which actual fracture surface is created in the material due to the action of externally applied loads. As noted earlier, the phenomenon of fracture can be approached from either a submicroscopic (atomistic) viewpoint, or from a macroscopic viewpoint. No attempt will be made here to detail such approaches, but rather a brief summary of the most common criterion will be outlined. Both approaches have merit, however, it is this author's opinion that a complete understanding of fracture cannot be attained without a viable physical model of the molecular processes involved.

The most commonly used macroscopic approaches for failure are those based on creating some type of "failure envelope" which where exceeded, predicts fracture and those based on extensions of the Griffith brittle fracture theory (24). The fracture envelope technique was developed for viscoelastic materials by Smith (25). In this approach, a fracture space is contructed for a particular material on the basis of itress, strain, and time as coordinate axes. Failure is then predicted whenever the $\sigma-\varepsilon-t$ state of the material lies beyond the boundary. 
The Griffith theory postulates the existence of small flaws within the material. In this approach, one writes an expression for the total energy as the sum of the elastic energy and the surface energy of the flaw. The resulting expressinn gives the total strain energy as a function of the stress state and the fracture stress is then defined as that stress at which the increase in surface energy due to a growing flaw is offset by the decrease in stored elastic energy plus any work added externally to the system. In viscoelastic materials, due to the ability of the material to transfer elastic strain energy to other than surface free energy, the Griffith approach must be modified. Rivilin and Thomas (26) and Williams (27) have pioneered much of the research in this area. Microscopic approaches for failure in polymers in the past have been wholly theoretical works. In most cases, the developers of these theories have been able to claim a certain amount of agreement with macroscopic experimental observations. It can be argued, however, that almost any not-too-unreasonable model might be fit to a set of macroscopic experimental data with the "correct" choice of material constants. However, there has been no experimental verification of these models at an atomic or molecular leve1, and therefore, it has been difficult to accept them with any degree of confidence.

Most microscopic theories of fracture have been kinetic theories that attempt to account in some way for time-dependent changes within the material which eventually lead to failure of the sample under consideration. These time dependent changes are related to and depend on the fluctuating thermal energy of the atoms, segments, or molecules. A review of some of the more recent of these theories is given by 
Kausch (28). Fracture criteria in these theories range from viscous flow and "slippage" of secondary bonds to primary bond scission and nucleation-growth of voids and flaws, depending on the class and structure of the polymers considered. Kaush notes that if we denote by $N$ the number of those elements (bonds, flow steps, nuclei, etc.) whose time dependent change is considered to determine the lifetime of the sample as a whole, then the general mathematical formulation of these theories can be represented by a rate equation:

$$
\frac{d N}{d t}=-N K(\sigma, N, T)
$$

where $K$ is the rate of breakage or nucleation, $\sigma$ the stress tensor and $T$ the absolute temperature. The interpretation of $K$ and its formulation is different in the kinetic theories of different authors according to the different statistico-mechanical mode1s.

As this study is limited to a very narrow and yet important class of polymers, i.e. the highly oriented semi-crystalline polymer fibers, further discussion of failure criteria will be limited to this group.

The micro-morphology of highly oriented fibers has been reviewed (Section 2.2) and arquments presented (Section 2.1) that backbone chain scission is the main mode of failure in many nolymers in this classificatime (11).

With the advent in 1964 (8) of EPR spectroscopy as a tool for experimental verification of microscopic chain scission, much interest has been generated in producing experimental data from which failure criteria can be developed and verified. In 196h, Zhurkov and Tomoshevskii (29), expanding ideas previously presented by Bueche (4), proposed using a Tobolsky-Eyring (3) type kinetic rate process to describe failure in 
nylon 6 and silk fibers. The basic equation for such a thermally activated stress-aided process is:

$$
\frac{d C_{B}}{d t}=\left\{w_{0} \exp \left[-\left(U_{0}-\gamma \sigma\right) / k T\right]\right\} \quad C_{U}
$$

where

$$
\begin{aligned}
& \frac{d C_{B}}{d t}=\text { rate of bond rupture } \\
& C_{u}=\text { number of unbroken bonds in cross-section } \\
& w_{0}, U_{0} \text {, and } \gamma \text { are kinetic rate constants commonly called the } \\
& \text { collision parameter, the activation energy, and the } \\
& \text { activation volume, respectively. } \\
& \sigma \quad=\text { stress } \\
& k=\text { Boltzmann's constant } \\
& T=\text { temperature }
\end{aligned}
$$

For constant temperature this equation reduces to:

$$
\frac{d C_{B}}{d t}=D \exp a \sigma
$$

where

$$
\begin{aligned}
& D=c_{u} w_{0} \exp \left[-U_{0} / k T\right] \\
& a=\gamma / k T
\end{aligned}
$$

Zhurkov and his co-workers used the applied macroscopic stress as $\sigma$ in equation (3) and presented experimental evidence which supported their theory for quasi-stress rate tests. In 1968, Roylance (30) completed an exhaustive study under the direction of K. L. DeVries, in which an effort was made to independently verify the work of the Russians. From this study, EPR bond rupture data was reasonably successful in correlating stress rate tests with the Zhurkov theory, although 
different kinetic rate parameters from those reported by Zhurkov were necessary in the Roylance characterization. Furthermore, it was found that theoretical predictions of other loading conditions, especially creep tests provided little if any correlation with experimental observations (31).

However, it was felt that reaction rate theory was well established and any model of failure should include its features but some modification seemed necessary to explain experimentally observed phenomena.

The key to the modification of such theory appeared to lie in the use of the stress term $\sigma$ and its relationship to the fiber microstructure. A careful look at equation (2) reveals that $\sigma$ is the atomic stress on a bond in a polymer chain and it is most likely that this stress is not equal (or proportionaly independent of time) to the applied macroscopic stress. In fact, as it is most unlikely that all chains in the polymer are equally stressed, there would exist a distribution of stresses related in some fashion to a distribution of effective stressed chain lengths in the fiber microstructure. This, major modification to the Zhurkov theory was suggested in 1969 by Roylance, et al (9); DeVries, et al (32); Peterlin (13), and Verma and Peterlin (33). Further development of the statistical mathematical approach to the problem was presented by Kausch and. Becht (34) and Kausch $(35,36)$ in 1970. A7so, it is interesting to, note that in 1970 , Chevychelov (37), a member of the Zhurkov group, reported developments along these same lines.

Even with the development of the above ideas, no exhausted study had been made to experimentally verify these theoretical concepts 
using a macro-micro-structure model. Such a model could be used to compare theoretically predicted stress, strain and bond scission results with those experimentally observed. It has been the objective of the author under the direction of $K$. L. DeVries to complete such a study. This dissertation reports the findings of this effort, part of which have recently been accepted for publication (38). 


\subsection{EPR FOUNDATIONS}

A thorough description of the theory and principles of Electron Paramagnetic Resonance spectroscopy are complex and require rather sophisticated quantum mechanics. As use of the EPR spectrometer and an understanding of the studies presented here does not require a detailed knowledge of the basic theory, only a brief and somewhat oversimplified description will be given here. A more detailed mathematical formulation of EPR theory can be found in several recent texts and publications $(30,39,40)$.

Electron Paramagnetic Resonance (EPR) spectroscopy is a type of absorption spectroscopy with principles and applications somewhat similar to those of nuclear magnetic resonance (NMR) and optical or infrared spectroscopy (IR). EPR spectroscopy is based on microwave absorption due to a Zeeman-type splitting of the energy levels of unpaired electrons in the presence of a magnetic field. Its physical basis is perhaps best understood by first considering the case of the free electron. Free or unpaired electrons in the presence of a magnetic field of strength, $H$, undergo an energy increase or decrease (Zeeman effect) depending on whether the angular momentum spin vector tends to line up with or against the magnetic field. The energy gap, $\Delta E$, between the two states is linear with the magnetic field, i.e. $\Delta E=g B H$ where $\beta$ is a universal constant called the Bohr Magneton, $g$ is called the spectroscopy splitting factor and is a constant that depends on the given environment associated with the free electrons. and $H$ is the magnetic field. This relationship might be represented graphically as shown in Figure 8 , where $M_{s}$ represents the spin 


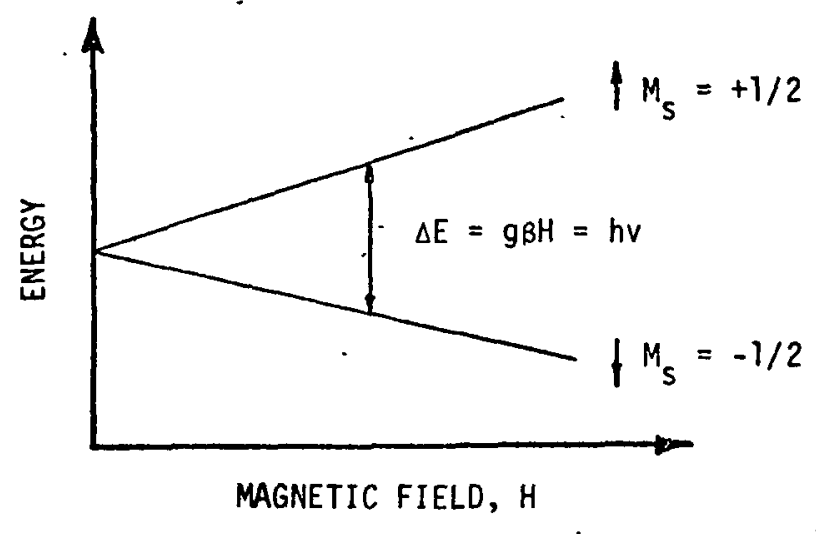

FIGURE 8. Zeeman splitting for a free electron

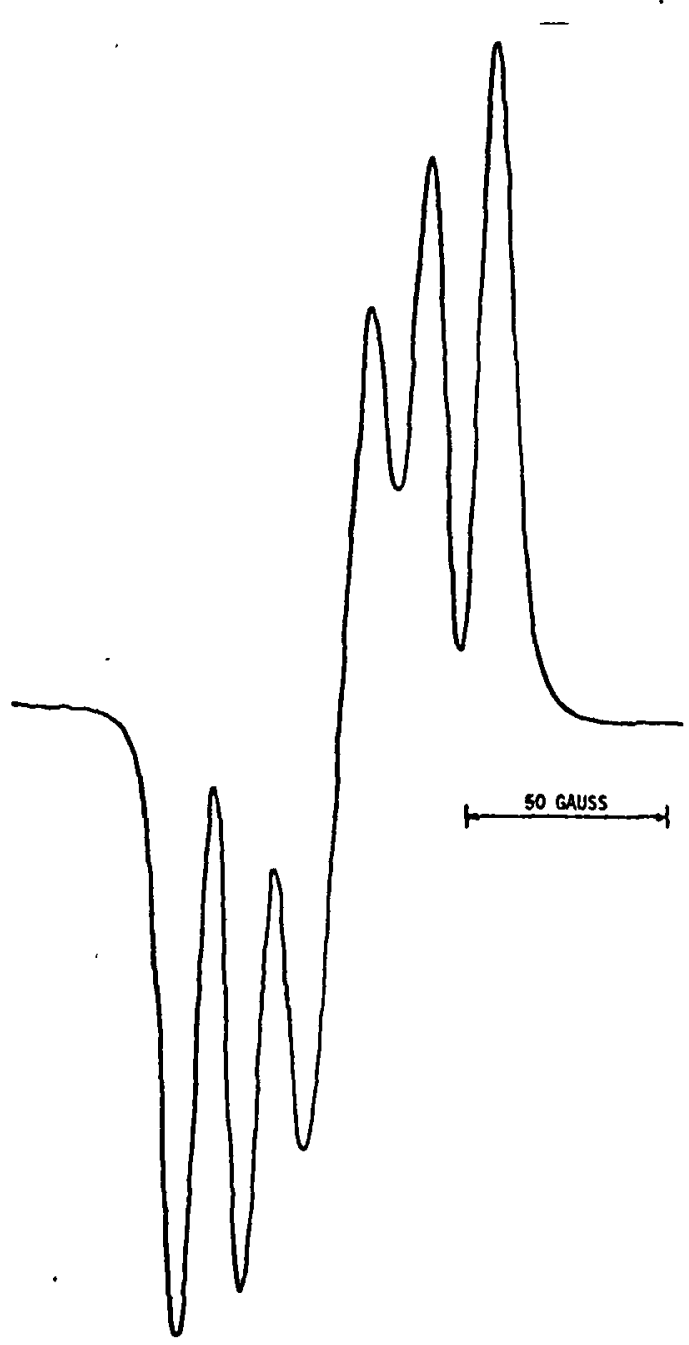

FIGURE 9. EPR spectra of NyTon 6 at room temperture 
quantum number of the electron. The population of the two energy levels at equilibrium are given by Boltzmann statistics and indicate that more of the free electrons will normally reside in the lower energy level or "ground state". By introducing incident radiation (for reasonably easily obtained magnetic fields of microwave radiation) of the appropriate frequency, $v$, such that the electromagnetic energy, hv, is equal to the energy difference between the two levels, $\Delta E$, it is possible to induce transitions between the two states, i.e.

$$
h \nu=\Delta E=g \beta H
$$

Electrons in the "ground state" absorb energy in being excited to the higher state and if those in the higher state give up energy to the lattice or surroundings in relaxing back to the "ground state", a net energy absorption takes place. The net absorption of microwave energy in inducing these transitions is detected through suitable electronics by the spectrometer and the output recorded (for elect:ronic conviences) in the form of the derivative of the absorption spectra. A typical spectra for nylon 6 is shown in Figure 9. The number of free electrons or free radicals contributing to a given spectra is given by the area under the absorption curve. In practice, the double integral of the recorded output gives the number of free radicals due to the spectrometer recording the first derivative of the absorption spectra. The shape of the absorption spectra includes such factors as hyperfine splitting and provides clues as to which unpaired electrons are present as well as how many. In systems more complicated than single electron, the unpaired electron senses not only the presence of the applied magnetic field, but is capable of 
both electrical and magnetic interactions with the nuclei and electrons of surrounding atoms. This interaction gives rise to the splitting that can often be used to identify the particular radical. Therefore, in principle, the EPR spectrometer is able to detect how many and which unpaired electrons are participating in producing the given spectra.

The applicability of EPR to fracture studies is limited to those materials in which bond rupture produces paramagnetic electrons (i.e. polymers such as plastics and rubber) in concentrations exceeding the experimental equipment limit $\left(\sim 10^{12}\right.$ unpaired electrons in the cavity). This requirement produces some limitations on the method, but the researchers at our laboratory as well as others have found EPR to be an extremely valuable analytical technique in the study of many diverse types of covalent bond rupture, $\gamma$-ray induced degradation, stress crazing, grinding, cutting, radiation of certain types of leukemia-induced blood cell rupture, and decay in teeth, to name a few. This study will present results on bond rupture in uniaxial stressed semi-crystalline polymers (in fiber form) with different loading histories. 
Page intentionally left blank 


\subsection{EXPERIMENTAL PROCEDURE}

\subsection{EXPERIMENTAL EQUIPMENT}

A Varian Associates, Model E-3 spectrometer with a regulated temperature controller was used as the basic element of this investigation. As shown in Figure 10, the spectrometer consists of two units. The first component, shown in the center of this figure consists of a magnet control unit, receiver, oscilloscope, system checkout and $x-y$ recorder. The second component, on the right of the figure contains a large field magnet, a klystron microwave generator, and a microwave resonance cavity. This portion of the unit is shown encompassed by the loading frame, load cell and sample loading apparatus used in the experimental tests. A summary of the performance characteristics of the Model E3 spectrometer as supplied by Varian Associates (41) is given is Table 1.

An important auxiliary unit in' the experimental work conducted using the EPR spectrometer is the specially constructed servo-controlled hydraulic loading system. The servo-controlled unit, shown on the left of Figure 10, is basically a feedback control system which regulates a hydraulic piston. The feedback for the system is supplied from either a strain-gage load cell (a Stratham Instruments Universal Cell and 1000 lb. load cell) or from a linear potentiometer coupled mechanically to the hydraulic piston. Test samples are connected to the load cell at one end of the loading frame, and attached to the hydraulic piston at the opposite end by brass connecting rods. The center section of the sample passes through the spectrometer microwave resonance cavity. Both load and displacement signals are amplified by a D.C. amplifier (Honeywe11 Accudata 120) and displayed on a Midwestern Instrument 


\section{Table 1}

EPR Spectrometer Performance Specifications

Operating Frequency

Sensitivity

Field modulation

RF power to Cavity

Klystron Frequency

Stability

Modulation Field Intensity

Scan Times

Magnetic Field Homogeneity

Field Control accuracy

Scan Range Accuracy

Scan Range Linearity

Field Resetability

Recorder Time Constants
8.8 to $9.6 \mathrm{GHz}$

Signal to average noise ratio is 60:1

$100 \mathrm{kHz}$

0.20 to $200 \mathrm{~mW}$

1 part in $10^{6}$

$5 \mathrm{mG}$ to $40 \mathrm{mG} \mathrm{p}-\mathrm{p}$

$18\left( \pm 0.25,0.5,1 G_{2}\right.$ with multipliers

$0.1,1.0,10,10^{2}, 210^{3}, 10^{4}$ )

Sufficient to resolve $70 \mathrm{mG}$ lines

$\pm 4 G+0.1 \%$ of dial reading

$\pm 0.2 \%$

$\pm 0.5 \%$ of max scan range

Within $0.1 G$

$0.1,0.3,1,3,10,30,100 \mathrm{sec}$ 
oscillograph located in the servo-controlled consol. The system can be set for either load or displacement feedback tests. During such tests, the hydraulic loading piston will follow and control from whichever electrical command is sent to the operational amplifier. Thus the servo-controlled hydraulic loading system allows real time observation of fracture by the spectrometer under almost any uniaxial loading condition. For example, with the aid of a small analog computer, test samples have been subjected to a wide range of programmed loads or displacements, including cylic ones up to $\sim 1000 \mathrm{cpm}$.

Temperature and atmosphere are controlled to the sample material in the spectrometer cavity by means of a modified Varian Associates variable temperature accessory and controller. This device automatically maintains the sample temperature at any value from $-160^{\circ} \mathrm{C}$ to $250^{\circ} \mathrm{C}$ with $\pm 3^{\circ} \mathrm{C}$ accuracy. The temperature at the sample is controlled by a stream of gas that has passed over an electric heater. For operation below ambient temperature, the gas flow is chilled in a heat exchanger with liquid nitrogen and then rewarmed by the electric heater to the desired value.

\subsection{SAMPLE MATERIAL, PREPARATION AND TESTING}

The major material used in this study was nylon 6 (polycaprolactam) fibers, supplied by Allied Chemical Company. These fibers were produced by a melt spun, hot draw process and were highly oriented in the as received condition. Their commercial destination would have been for use as high-strength tire yarn. Two different types of nylon 6 fibers were studied. Nylon $6 \# 1$, having a number average molecular weight of 25,000, a single filament diameter of 0.00100 inch with 202 filaments plied together in each yarn. 
Nylon 6 \#2, (Allied R-0245) having a number average molecular weight of 30,000 , a single filament diameter of 0.00105 inch with 136 filaments plied together in each yarn.

A few preliminary tests were also conducted using nylon 66 fibers, supplied by E. I. DuPont de Nemours and Company, Inc. designated as T714, code 840-140-T20-714. These fibers had a single filament diameter of 0.00108 inch with 139 filaments per yarn.

Tensile samples were prepared in such a manner that fracture would occur in the EPR cavity and not occur at the sample "grips". Essentially, a tensile "bar" was made by winding the yarn on a jig to the desired length and number of yarn strands. Then the yarn strands were tied at four places and bonded* between the ties as shown in Figure 11. After the epoxy was cured at room temperature, a predetermined number of strands were cut and removed from the center portion of the sample. The procedure insured that the sample would fracture in the center portion, much as a necked or notched solid tensile bar. This technique greatly improved the quality of the EPR measurements, as the fracture process could be observed in the EPR cavity from its microscopic beginning to its macroscopic catastrophic failure point. Figure 12 shows an actual sample before and after fracture.

A11 samples were eleven inches in total length. Each bonded area was approximately 1.8 inches long. Two basic winding and cutting patterns were used. For nylon $6 \# 1$, twenty-five strands were wound and ten

\#Resiweld 7002 Epoxy, H. B. Fuller Company; Part A (Resin), part B (hardener). Bonding mixture: 1 part A to $21 / 4$ part $B$ by weight. 


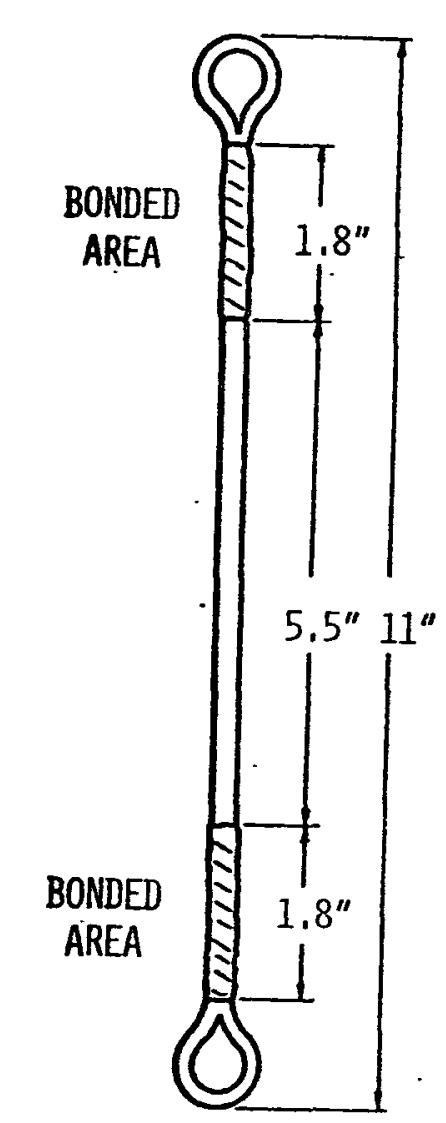

FIGURE 11. Typical nylon 6 and nylon 66 test sample configuration and dimensions. 
cut after bonding (Figure 13). This gave a sample area of $2.38 \times$ $10^{-3}$ in. $^{2}$ and an effective EPR cavity volume of $1.535 \times 10^{-2} \mathrm{~cm} .{ }^{3}$. For nylon 6 \#2 and nylon 66, thirty-five strands were wound and thirteen cut after bonding (Figure 14). For nylon $6 \# 2$, this resulted in a sample area of $2.57 \times 10^{-3}$ in. $^{2}$ and an effective EPR cavity volume of $1.66 \times 10^{-2} \mathrm{~cm}^{3}$. The same parameters for nylon 66 were; area $2.82 \times 10^{-3}$ in. $^{2}$ and volume $1.82 \times 10^{-2} \mathrm{~cm}^{3}$.

Nylon materials are humidity sensitive and because the absorbed water acts like a plastizer, it is important that this property be controlled to obtain meaningful comparative results. All nylon samples were dessicated* for one to two weeks before test.

Testing of the samples was accomplished by mounting the sample such that the center portion passed through the EPR cavity and was enclosed in a atmosphere-temperature controlled quartz tube. The ends of the sample were then attached to the loading grips, the temperature and atmosphere set and the sample extended to fracture according to a predetermined mode of loading. Modes of loading included constant strain rate, constant strain (stress relaxation), step. strain, constant stress rate, constant stress (creep), and several frequencies of cyclic stress fatigue. Temperature tests ranged from $-25^{\circ} \mathrm{C}$ to $+100^{\circ} \mathrm{C}$. It was found that the type of atmosphere and the temperature was very important in controlling the decay rate of the free radicals generated during fracture. Further comments will be made on this later, however, to obtain minimum decay rates a 11 tests were conducted in a dry helium a tmosphere.

\#In phorphorous pentoxide $\left(\mathrm{P}_{2} \mathrm{O}_{5}\right)$. 


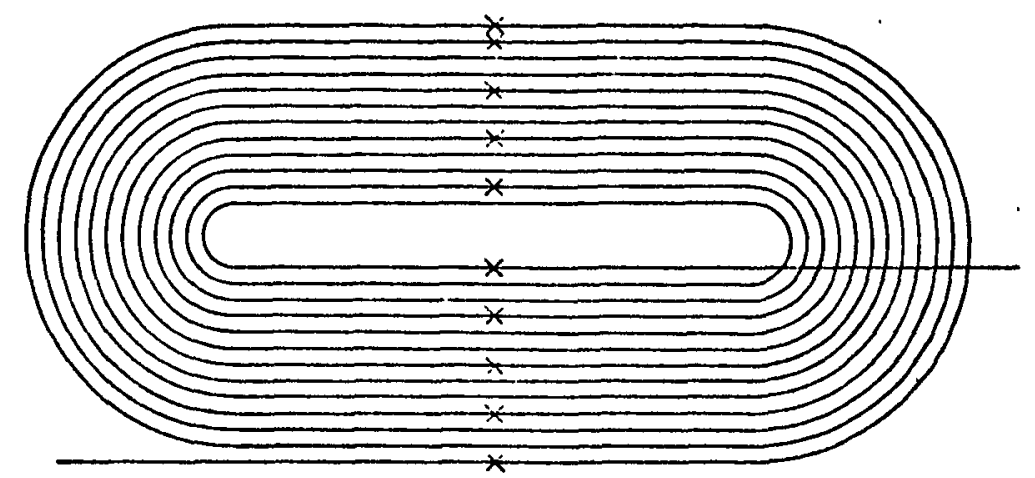

FIGURE 13. Typical winding and cutting pattern for. nylon 6 \#1 ( $x$ denotes strands to be cut after bonding)

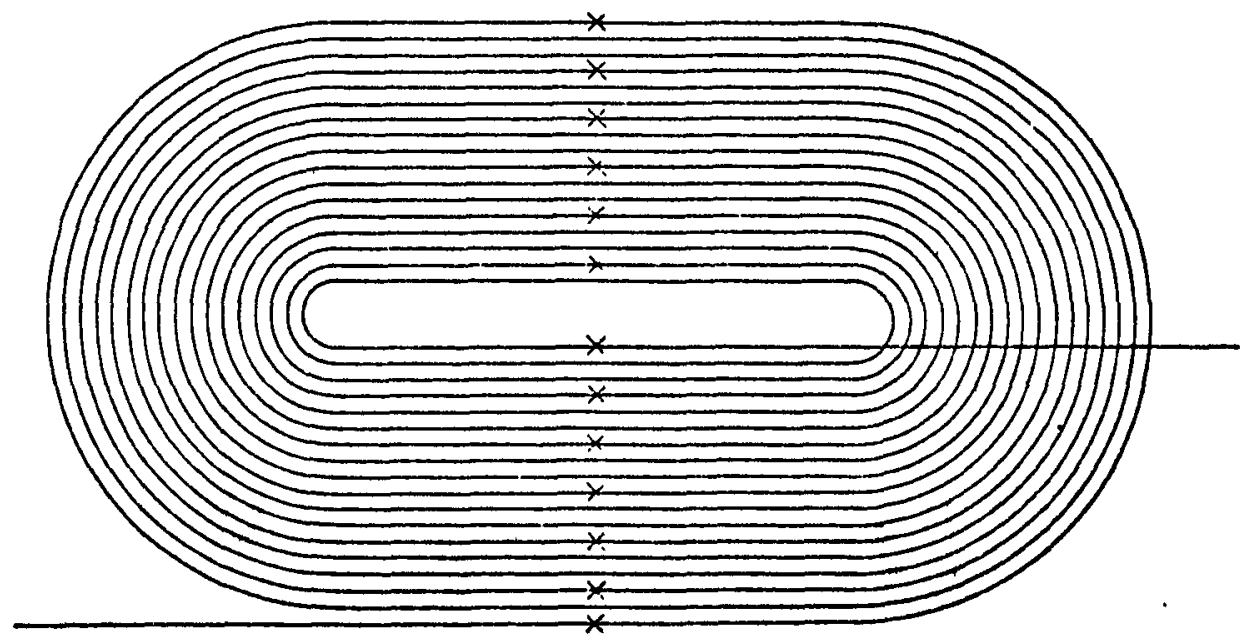

FIGURE 14. Typical winding and cutting pattern for nylon $6 \# 2$ and nylon 66 ( $x$ denotes strands to be cut after bonding) 
As mentioned previously, the number of free radicals or spins is proportional to the area under the absorption curve (the double integral of the recorded output).

Given an EPR spectrum, a number proportional to the number of spins present can be found by numerical double integration. With the aid of a standard sample containing a known number of stable spins, the result of this double integration can be converted to the actual number of spins in the cavity at the time the spectrum was recorded. The standard sample used in this work is one commerically available from Varian Associates, referred to as the "strong pitch", and contains $3 \times 10^{15}$ spins per $\mathrm{cm}$ of effective cavity length. The calculation of the number of spins involves comparing the double-integration result of the test samples spectrum with that of the standard sample. This comparison is strictly valid only if the test sample and the standard sample are scanned under spectroscopically identical conditions: microwave power, scan width, filling factor, modulation amplitude, and time constant. It is often impossible for the standard and the sample to have the same filling factor--they may simply be different in size; this factor--plus the inherent uncertainty in the standard (25 per cent in this case)--limit the accuracy of the number of spins to $\sim 50$ per cent even in the best of cases (39). The equation giving the number of spins in the cavity of the test sample is:

Test Sample
Spins in Cavity $=\frac{(\text { Pitch Sample Std Value)(Eff Cavity Length) (Test Sample Area) }}{\text { (Pitch Sample Area)/(Pitch Sample Gain) (Test Sample Gain) }}$

where the effective cavity length is $1 \mathrm{~cm}$ if the sample uniformly fills the entire cavity length. To obtain the spins $/ \mathrm{cm}^{3}$ in the sample the 
above spin number is divided by the sample volume, based on an effective cavity sample length of $1 \mathrm{~cm}$. It is impractical to record the entire spectrum for future double integration during a time-dependent loading test; the kinetics will not stand still while the spectrum is scanned. Fortunately, there is a direct proportionality between the value of a spectrum's double integral and the height of any one of the peaks on the first derivative presentation. This proportionality was determined numerically for nylon at various temperatures by the following procedures: at each of several temperatures ranging from $-25^{\circ} \mathrm{C}$ to $+100^{\circ} \mathrm{C}$, the sample was subjected to stepwise loading so as to produce a family of spectra as in Figure 15. Each of these spectra were then integrated numerically and the corresponding number of spins computed (taking into account the temperature-dependence of the pitch sample). The number of spins was then plotted against the height of a prominent peak in the spectrum. A line was fitted to these points using a least-squares criterion. The result of all this was a series of straight-line equations, one for each temperature, giving the number of spins as a function of the height of the peak. Figure 16 shows this relationship for nylon 6 fibers at room temperature $\left(22^{\circ} \mathrm{C}\right)$.

With the above relations avallable, it is not necessary to scan the entire spectrum in order to monitor the spin concentration. The spectrometer is tuned to "sit" on the peak chosen so that the electrical output of the spectrometer's receiver is a measure of the height of that peak. Taking into account the gain setting of the spectrometer receiver, the spectrometer output can be recorded osclllographically as a function of time and this record then reduced and scaled to read directly in spins per $\mathrm{cm}^{3}$ of sample. Even though the absolute magnitude 


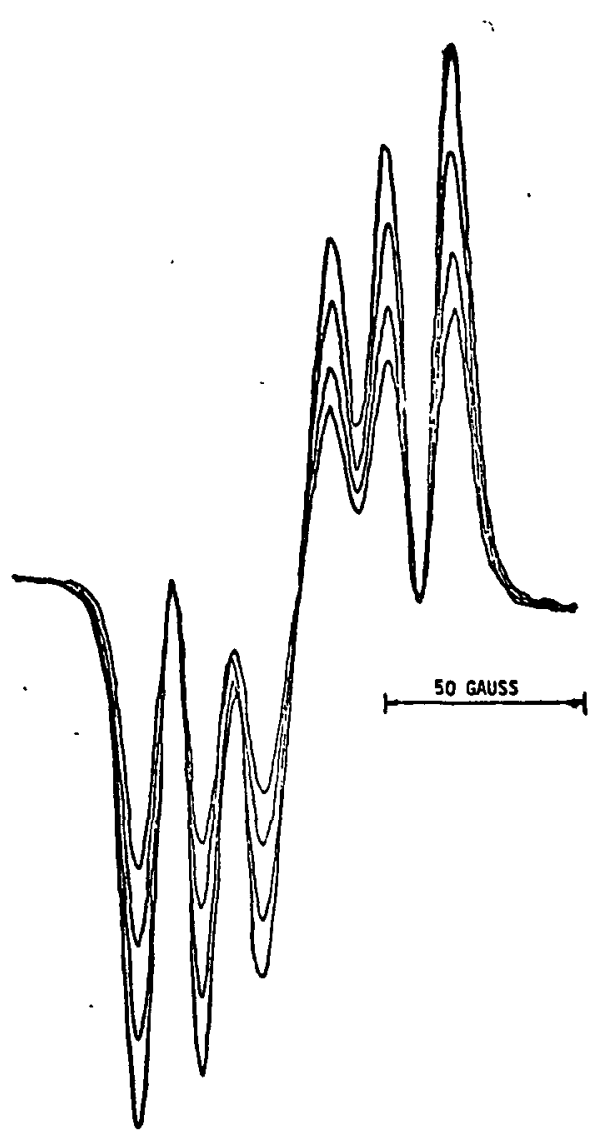

FIGURE 15. Nylon 6 spectra family produced by stepwise loading of sample

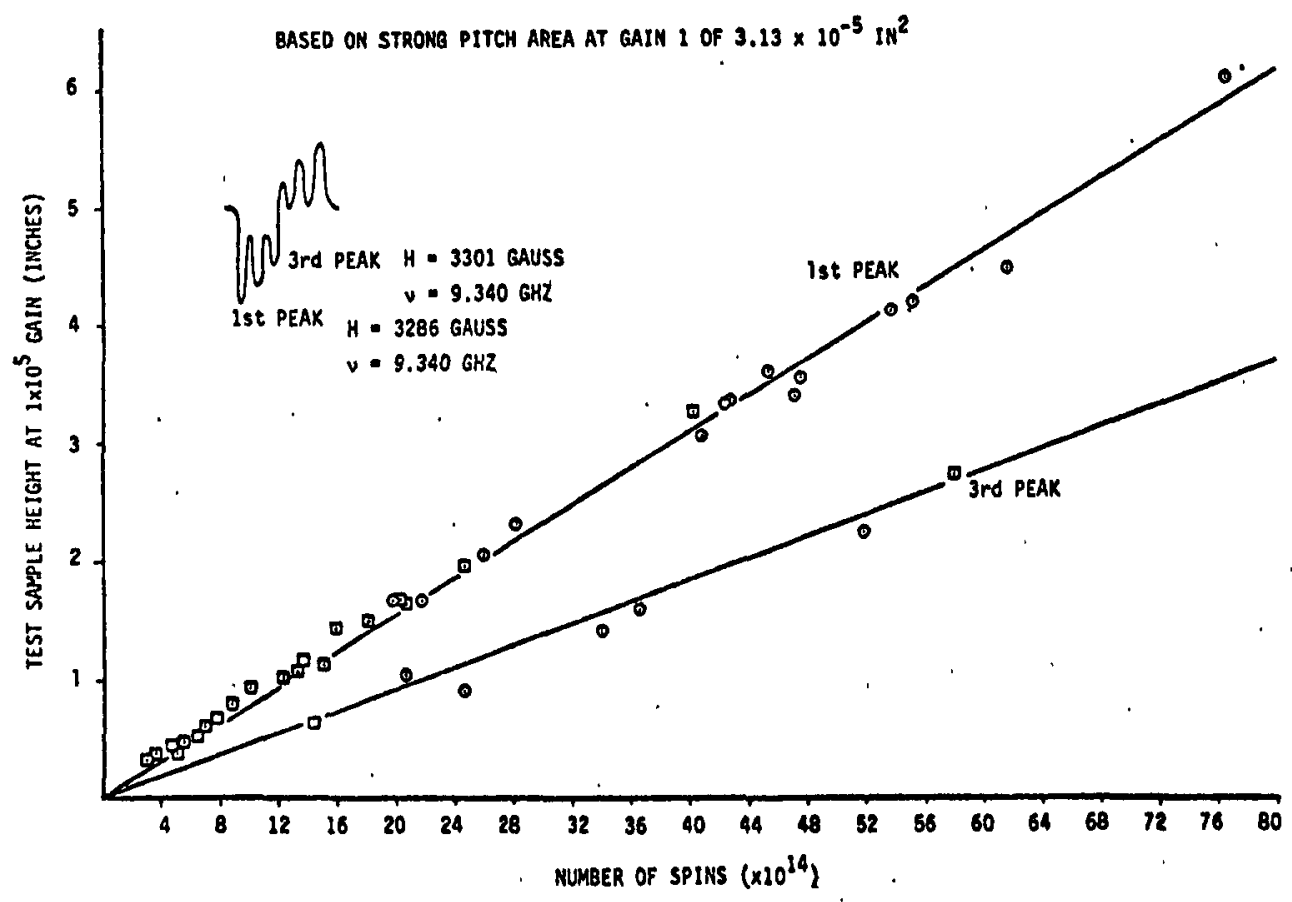

FIGURE 16. Nylon 6 and nylon 66 calibration curve at room temperature 
of the spin concentration may be in error by as much as 50 per cent as noted above, the kinetics themselves are not distorted. In other words, the shape of the curve is accurate even thaugh the position of the vertical axis may be in error. 


\subsection{THEORETICAL AND EXPERIMENTAL RESULTS}

\subsection{INTRODUCTION}

It is important at this point to establish the basic concepts that encompass the authors research in somewhat more detail than in the previous sections.

First, the goal of this study was to establish an atomistic failure criterion for a class of highly-oriented semi-crystalline polymers in fiber form, specifically nylon 6 fibers, that would relate microscopic or atomic occurences to the macroscopic behavior and properties of the material.

Second, the approach followed in accomplishing this goal was to first establish an idealized mathematical model to represent the particular polymer fiber structure and to select a particular failure criterion. Also, a wide variety of experimental tests were completed that simultaneously recorded stress, strain and bond scission as functions of time. Experimental and theoretical results were then compared to establish the validity and weaknesses of the mathematical model and failure criterion. As the author is not a morphologist, the mathematical model was established by taking the macro-micro structural parameters for highly-oriented semi-crystalline polymer fibers as reported by knowledgeable researchers and constructing an idealized physical model from which macroscopic loadings could be related to the micro-structure. The failure criterion chosen to predict bond scission was based on Tobolsky-Eyring (3) rate process or reaction rate theory. The experimental data was obtained using the EPR spectrometer in conjunction with the servo-controlled loading appara- 
tus. Theoretical results were obtained by numerical methods using a Univac 1108 computer.

Third, the study was 'limited to a very narrow and yet important class of materials; the highly oriented semi-crystalline polymer fibers. Nylon 6 fibers were chosen to represent the class of materials due to the large EPR signals obtained during the faflure process which seemed to indicate backbone polymer chain scission was the main mode of failure rather than unwinding or slippage caused by secondary bond failure. (EPR spectroscopy does not detect secondary bond failure).

Uniaxial tensile loadings were the only modes used in testing the fibers. Loading rates were relatively high (failure occuring in less than five minutes) and only short time creep tests were observed (less than 30 minutes). The basic reasons for limfting testing to short time failures were: (a) to minimize the viscoelastic properties of the material, which are thought to dominate in long time failure modes; (b) to minimize decay of the free radicals observed during the fracture process.

Tests were conducted over a limited temperature range only and mostly below the $\mathrm{Tg}$ value of nylon 6 fibers. Also, the effects of water as a plastizer were not studied but water content was controlled by sample dessication.

Fourth, as the study culminates three years of research, a large amount of data has been accumulated, i.e. over. 350 fiber samples have been tested and more than 300 computer runs have been generated. Clearly, this produces a need for selectivity as to how much data should be included here. In general, only representative 
data and their qualitative consequences will be presented; the rest will be placed in the laboratory files.

\subsection{MATHEMATICAL MODEL}

The micro-structure models of highly-oriented semi-crystalline polymers have been reviewed and although these differ in some important details, they all contain a basic common element, that is, all include an alternating structure of ordered or semi-ordered and disordered amorphous or tie molecule regions. Some researches $(13,15-21)$ maintain these ordered regions are truly crystalline regions alternating with amorphous regions (Figures 3 and 4) while others $(14,23)$ accept a paracrystalline or sloppy lattice concept where only degrees of order exist (Figures 5 and 7 ). In most of these models, the highly ordered regions of the polymer are alternately tied together by disordered or amorphous phases of the polymer. An effort has been made by several researchers to determine in which part of the micro-structure chain scission occurs during mechanical loading. Verma and Peterlin (33) observed that the EPR spectrum shape of free radicals generated by $\gamma$-radiation (which indiscriminately breaks bonds) of nylon 66 fibers was orientation sensitive, whereas the spectrum obtained from mechanical stretching did not show any conspicuous anisotropy properties. From this they concluded that the majority of the free radicals generated during the $\gamma$-radiation were contained in the highly ordered or crystalline regions of the polymer, whereas those generated during mechanical loading were located in molecular chain sections with little or no orientation, i.e. they were located in the quasi-amorphorous layers sandwiched between the crystal blocks. Further evidence supporting the basic assumption 
that during mechanical loading chain scission occurs almost wholly in the amorphous or tie molecule regions was reported by Becht and Fischer (42). In their experiments, chain scission was induced in nylon $\sigma_{i}$ fibers by $\gamma$-radiation and by mechanical loading. The samples were then swollen in methacrylic acid and the EPR spectrum between the samples compared. The methacrylic acid is known to penetrate and swell only the amorphous regions of the polymer and reacts with any free radicals present to form a polymerization radical of the methacrylate acid. As a result of the comparison, it was found that a mixed EPR spectrum was obtained in the $\gamma$-radiated sample whereas only the polymerization radical of the methacrylic acid was observed in the mechanically loaded sample. The results of these studies are in basic agreement with observations by Backman (43) in studies at this laboratory. Samples of polyethylene and nylon that were $\gamma$-radiated produced free radicals with a much greater stability than those produced by mechanical degradations. The different rate of decay of the original free radicals to a peroxy type radical was attributed to the ability of oxygen to diffuse into the crystalline and amorphous portions of the polymer at different rates. It was therefore concluded that mechanical degradation was occurring mainly in the amorphous regions of the polymer.

An important point to make here is that although chain scission is thought to occur almost wholly in the disordered amorphous or tie molecule regions of the polymer fibers there still remains the question: does failure occur in every tie molecule region or are there specific critical tie molecule or flaw regions distributed throughout the fiber in which failure predominates? Observation of the number of free 
radicals generated during fracture has substantiated the concept of a failure process occurring throughout the fiber at many critical flaw regions (30) and analysis of this number in terms of the total amorphorous chain scissions that could occur seem to indicate only a fraction of the possible are generated and/or observed by EPR (44). Further discussion of this point will be made later, however, there exists some strong evidence from this study and others favoring the concept of regions acting like rheological units or "crystalline blocks" interspaced with "critical amorphous flaw" regions to explain the strength and failure properties of the polymer fibers. These "crystalline block" regions may in fact contain some amorphous or tie chain regions and yet act elastically as a unit. The concept of a critical amorphous flaw region in which failure predominates i.e. a region that would perhaps contain the fewest and the most poorly distributed load bearing tie molecules, is in basic agreement with previous observations in this laboratory $(9,30,43,45)$. In fact, EPR studies by Backman and DeVries (45) of the number of free radicals formed during cutting and grinding of the bulk semi-crystalline polymers indicate that failure occurs in flaw regions following a selective path of "least resistance" around the crystalline blocks. The basis for this conclusion was that only approximately two per cent of the possible chains per $\mathrm{cm}^{2}$ in a crystalline lattice were observed to undergo chain scission during failure. The exact number of chains per $\mathrm{cm}^{2}$ in the ordered and the disordered regions of the polymer are not known and furthermore that portion of the total amorphous chains that are load bearing tie chains can only be speculated. An additional important concept that earlier models neglected in 
establishing a failure criterion, was the existance of a distribution of stresses within the polymer fiber related in some fashion to a distribution of effective stressed chain lengths in the critical flaw regions. This concept of amorphous regions consisting, in part, of a set of tie chains, all of different length and number was recently proposed by several authors $(9,13,33-37)$ and was essentially the key to the success of this study. The first experimental verification of such a distribution was reported by Kauch and Becht (34) where bond scission data from EPR step strain tests was plotted in the form of a histogram giving the average number of chain scissions versus the strain increment (chain length).

In similar tests of our laboratory the sample was loaded to the point just before bond breakage was detected. The strain was then stepped in equal increments and the number of bonds broken during an increment of extension recorded. Figure 17 shows the results of step strain room temperature tests of two different nylon 6 samples plotted as histograms.

It should be noted that due to the fact that the sample fails rather catastrophically, the tailing side of the distribution is missing. These experimental studies, therefore, give no information on the distribution in this region.

Analyzing these results in terms of a mathematical distribution function, it was necessary to assume some type of form to the missing portion. It was decided from analys is of the data, some of which gave considerable portions of the talling side, that a symmetrical distribution was the most probable type. With this assumption, the observable portion of the results were plotted on probability paper 


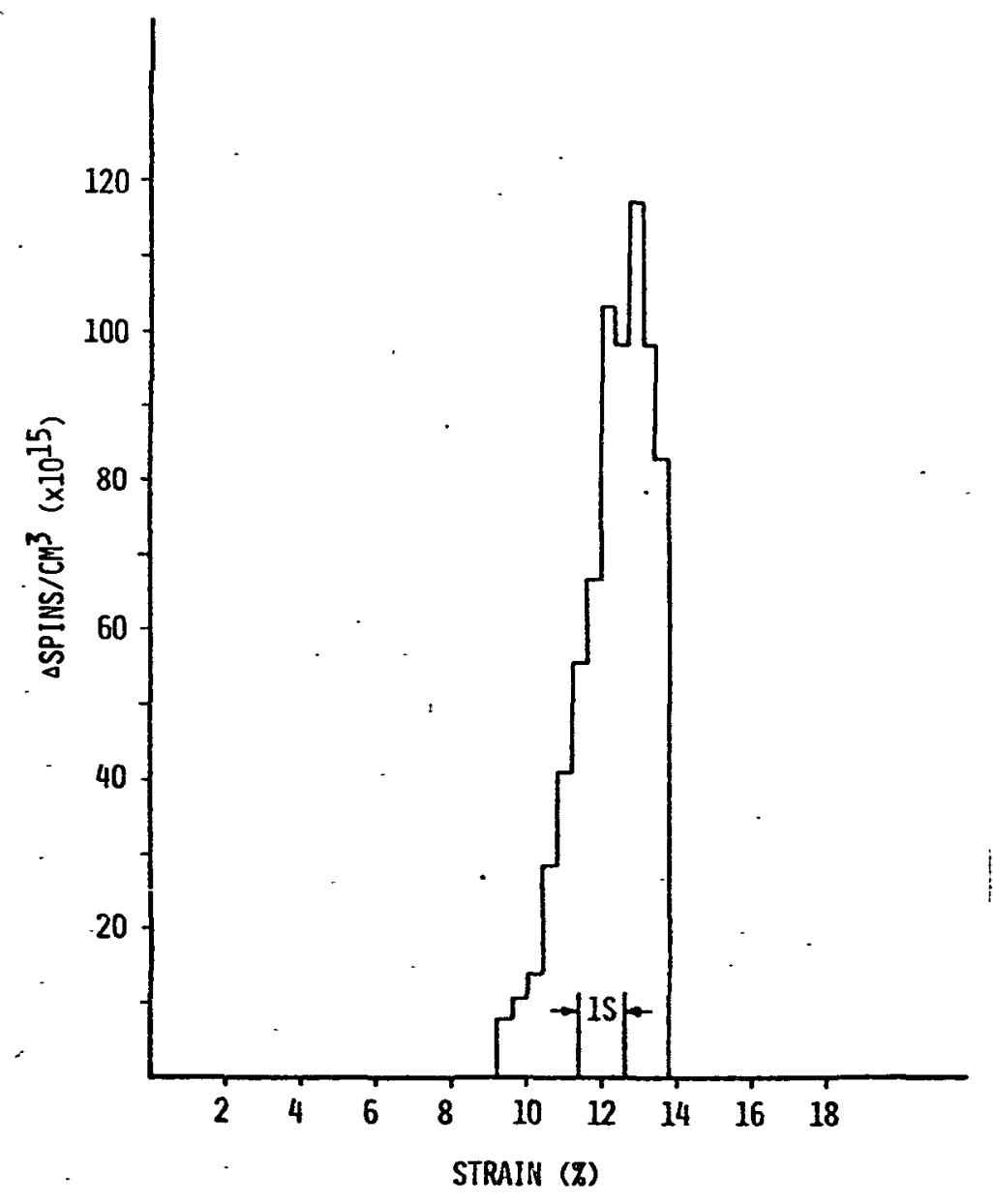

a. Nylon 6 \#1

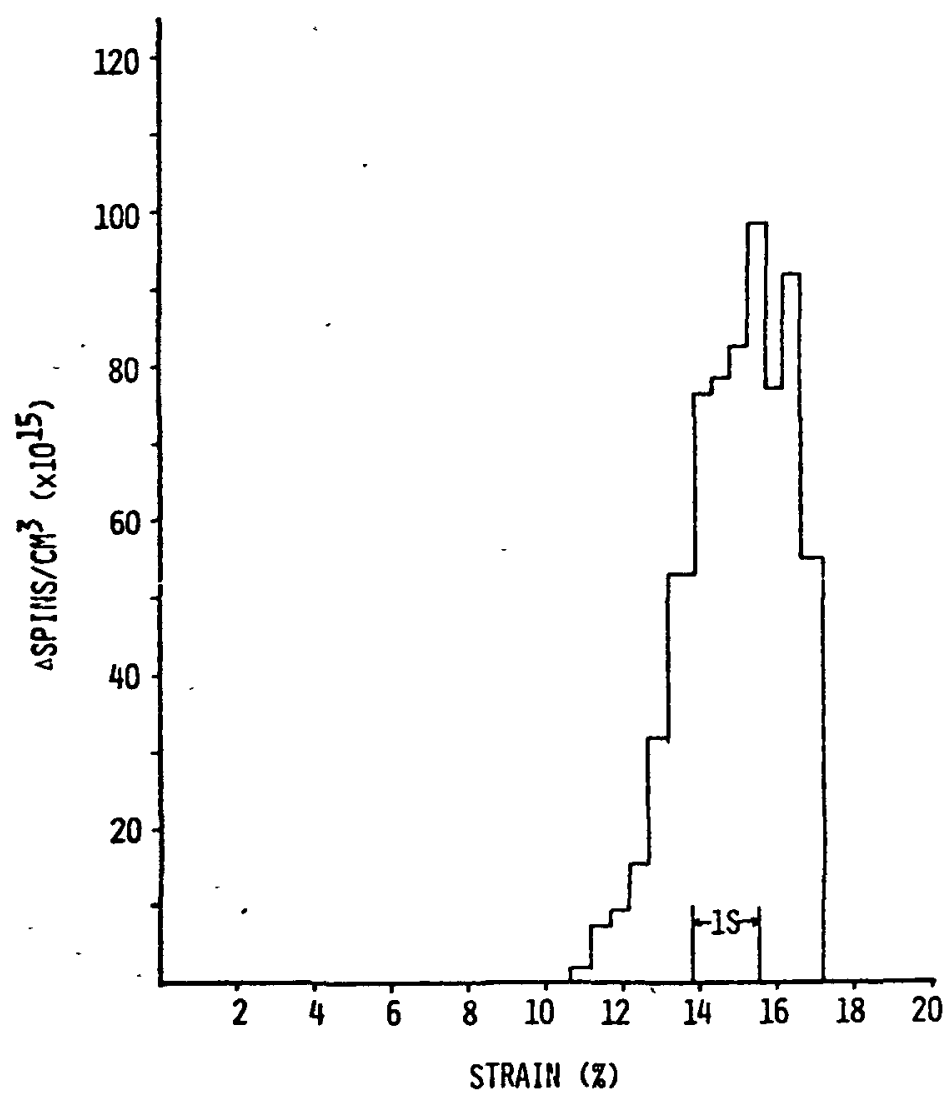

b. Nylon 6 \#2 annealed.

FIGURE 17. Histograms of nylon 6 samples from step strain data at room temperature. 


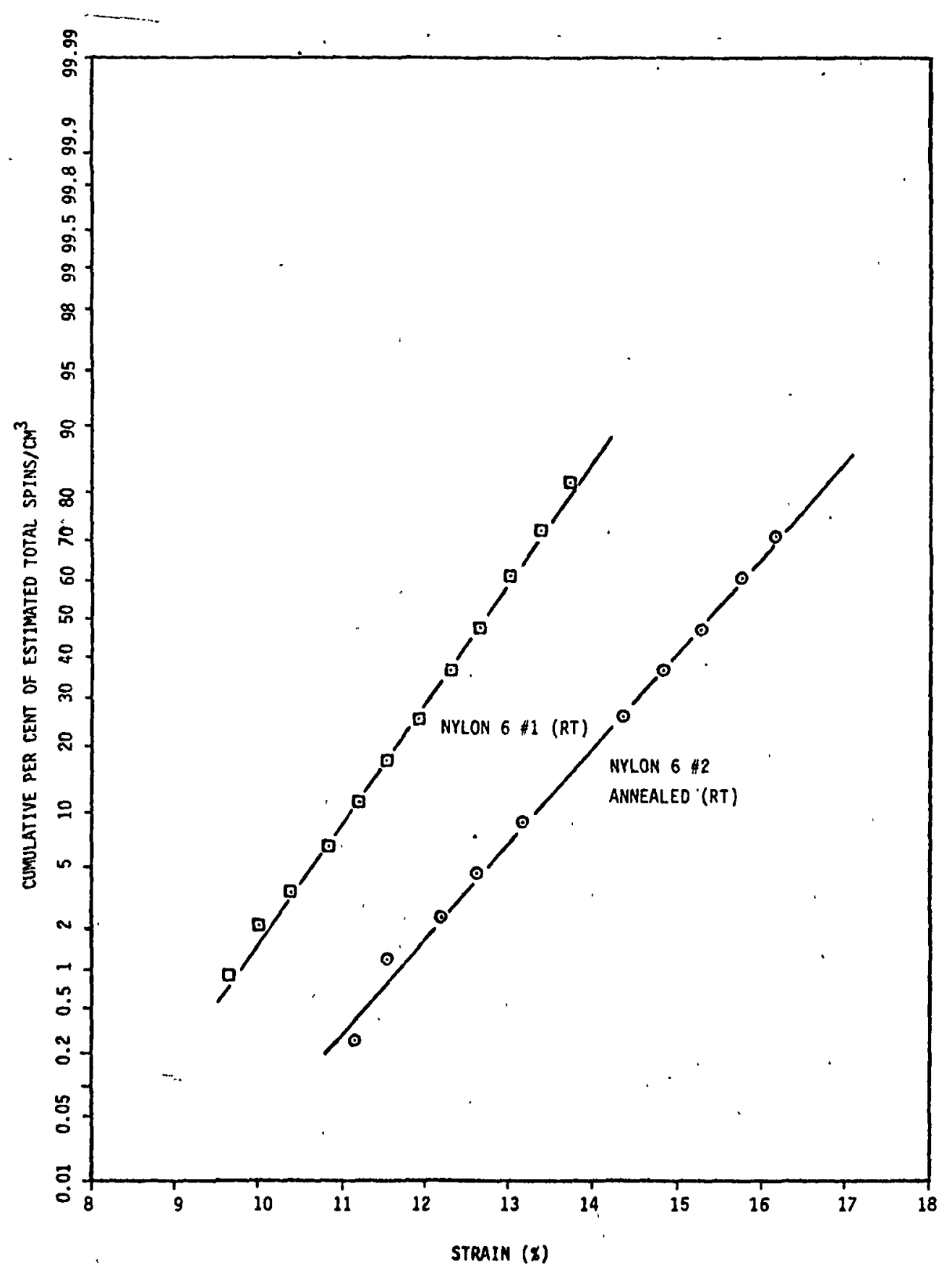

FIGURE 18. Probability plot of nylon 6 histogram data 
and this portion, at least, was found to comply with a normal or Gaussian distribution criterion. Figure 18 shows the results of the histograms shown in Figure 17 plotted on probability paper. The criterion for a Gaussian distribution is that these plots be straight lines. From these results an "apparent or effective distribution" in length was found to exist and was relatively narrow with a one standard deviation value of about ten per cent of the distribution mean strain for nylon 6 \#1 at room temperature. This "apparent or effective" distribution could have been due, in part, to macroscopic differences in fiber lengths created during manufacturing filament winding or during sample preparation. However, at least two items supported a microscopic effect. Samples tested had an initial length of eleven inches. A one standard deviation of ten per cent would mean a total distribution width of about thirty per cent. This would indicate fibers with over three inch differences in length in order to account for the distribution from purely macroscopic effects. Extreme care was taken in sample preparation to insure equal lengths of the sample fibers and although small length differences undoubtedly were present, none would be expected to exceed even a few tenths of an inch. Secondly, evidence of a microscopic distribution was recently obtained in this laboratory during creep tests on highly drawn nylon monofilament rods. EPR results from such tests indicated a bond breakage curve very similar in shape to that obtained in creep tests for nylon 6 fibers. As was noted, theories not incorporating a distribution of stresses due to the material microstructure have been very unsuccessful in predicting bond breakage in creep tests (31). Analyzing the above experimental evidence, it was decided that 
any idealized model which was proposed to explain the failure mechanism in oriented fibers should include as a minimum the following two parameters:

1. A block type structure of alternating ordered rheological units and disordered or amorphous critical flaw regions.

2. Critical flaw regions composed, in part, of a number of tie chain molecules that are distributed according to their various lengths as links between the ordered crystalline block rheologịcal units.

The mathematical model proposed will use a basic alternating sandwich structure of crystalline blocks and critical amorphousflaw regions. These regions will have no predetermined size and will be related to each other by ratio values only. A distribution in effective length of chains in the critical flaw regions will be included. Such distribution in tie chains must change as bond rupture occurs and the model will include faculties to account for the change in distributions with stress and time. The failure criterion proposed to predict chain scission will be a Tobolsky Eyring (3) type rate process or reaction rate theory $i$. e. a stress aided activation energy process, having the form:

$$
\frac{d C_{u}}{d t}=-k C_{u}
$$

where

$$
\begin{aligned}
& k=w_{0} \exp \left[-\left(U_{0}-\gamma \sigma\right) / k T\right] \\
& c_{u}=\text { number of unbroken bonds in the critical cross section } \\
& \sigma=\text { stress on each bond in the critical cross section }
\end{aligned}
$$




$$
\begin{aligned}
& K=\text { Boltzmann's constant } \\
& T=\text { absolute temperature }
\end{aligned}
$$

with kinetic rate constants

$$
\begin{aligned}
& w_{0}=\text { collision parameter } \\
& U_{0}=\text { activation energy } \\
& \gamma=\text { activation volume }
\end{aligned}
$$

Figure 19 can be visualized as the idealized model of the test 'sample where the microstructure is represented as repeated average "crystalline-block" and "critical flaw" regions. The model takes one such crystalline block and flaw region as representative of the average of all critical regions of the fiber. Figure 20 is an idealized representation of this average region. The ordered or "crystalline block" region is represented by a number of aligned polymer chains each with an average bond area $A_{b}$. The disordered or "critical flaw region" is shown to be composed of sets of tie chains. Each of these " $n$ " sets has a different effective length and number of chains. according to a prescribed type of distribution. The original length of the flaw region is $\ell_{o a}$ and is initially considered to be taut whereas all other sets may be kinked, disoriented, folded, etc., and do not become taut until the sample is strained to the point where that particular set reaches its effective length. For convience in visualizing the geometry involved in the mathematical derivation, these sets are shown in their extended configuration.

From the geometrical relationships indicated in Figures 19 and 20 , the macroscopic loadings were related to the micro-structure of the of the representative unit shown in Figure 20. Derivation of the governing equations for a macroscopically applied stress $\sigma_{T}$ and 


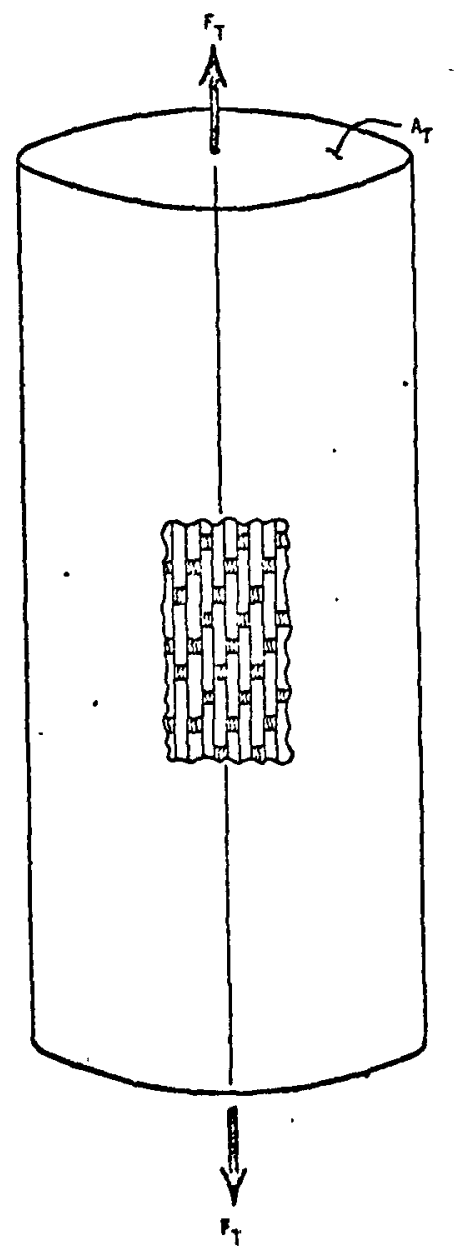

FIGURE 19. Idealized mode1 of fiber structure showing microscopic block regions

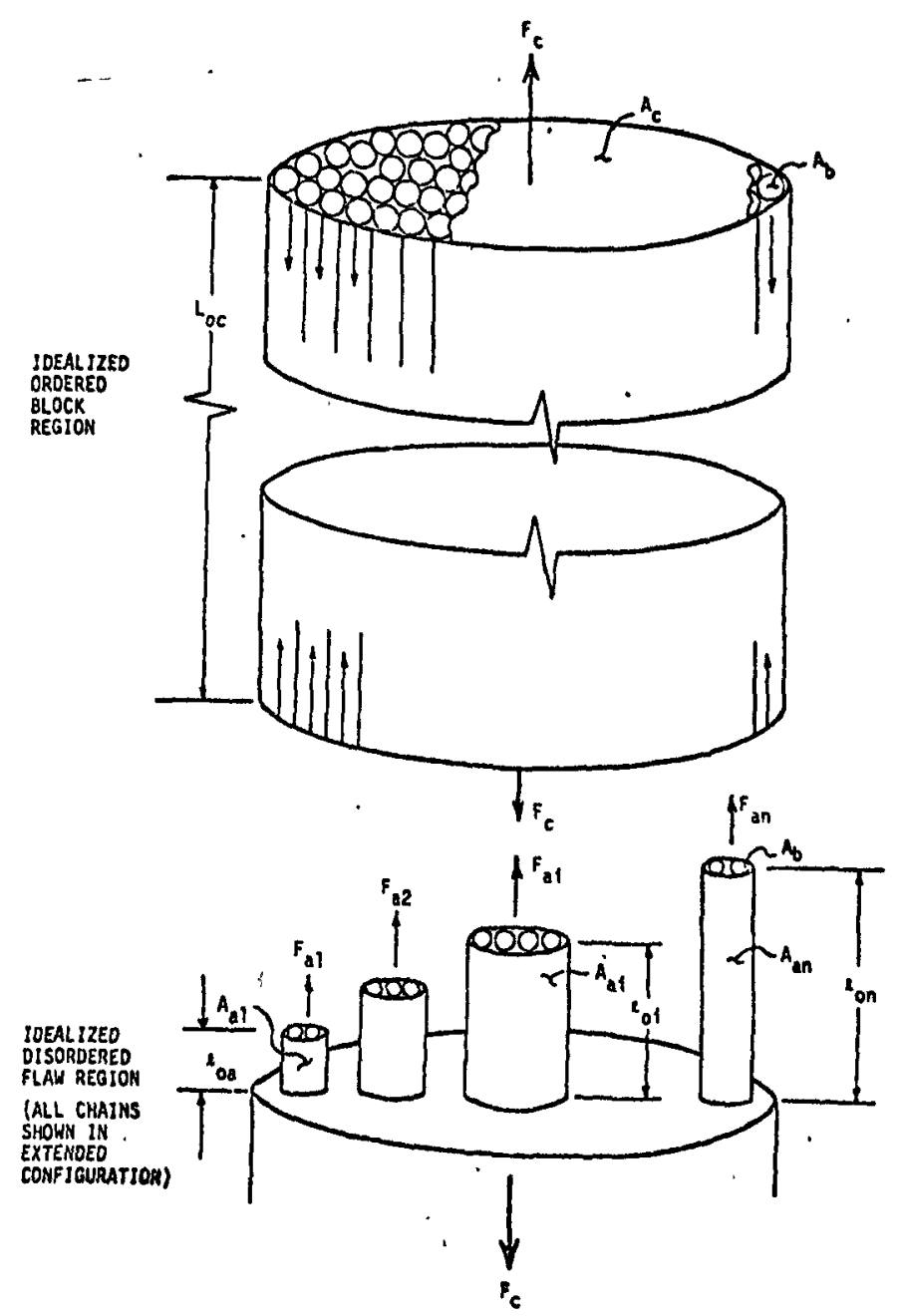

FIGURE 20. Average "crystalline block" and "critical flaw" region of idealized fiber microstructure 
also for a macroscopically applied strain $\varepsilon_{T}$ follows:

Definition of terms (see Figures 19 and 20)

$$
\begin{aligned}
& \sigma_{T}(t)=\text { macroscopic stress } \\
& F_{T}(t)=\text { macroscopic load } \\
& A_{T}=\text { macroscopic area } \\
& \varepsilon_{T}(t)=\text { macroscopic strain } \\
& \delta_{T}(t)=\text { macroscopic change in length } \\
& L_{O T}=\text { macroscopic original length } \\
& M \quad=\text { number of average critical regions }
\end{aligned}
$$

$\sigma_{c}(t)=$ stress in the "cry-blk" region

$F_{c}(t)=$ force in the "cry-blk" region

$A_{c}=$ original area of "cry-blk" region

$\varepsilon_{c}(t)=$ strain in the "cry-blk" region

$\delta_{c}(t)=$ change in length of "cry-blk" region

$L_{\text {oc }}=$ original length of "cry-blk" region

$c_{0} \quad=$ original number of polymer chains in "cry-blk" region

$\begin{aligned} & A_{b}= \text { average or effective area of a single polymer chain } \\ & \text { in "cry-bik" region }\end{aligned}$

$E_{b} \quad=$ modulus of elasticity of a single polymer chain

$\sigma_{j}(t)=\begin{aligned} & \text { stress on } i^{\text {th }} \\ & \text { region. }\end{aligned}$

$F_{a j}(t)=$ force on $i^{\text {th }}$ set of bonds in flaw region

$A_{a j}(t)=$ area of $i^{\text {th }}$ set of bonds in flaw region

$\begin{aligned} \varepsilon_{i}(t)= & \begin{array}{l}\text { strains on } i^{\text {th }} \\ \text { flaw region }\end{array}\end{aligned}$

$\delta_{i}(t)=$ change in length of $i^{\text {th }}$ set of bonds in flaw region 


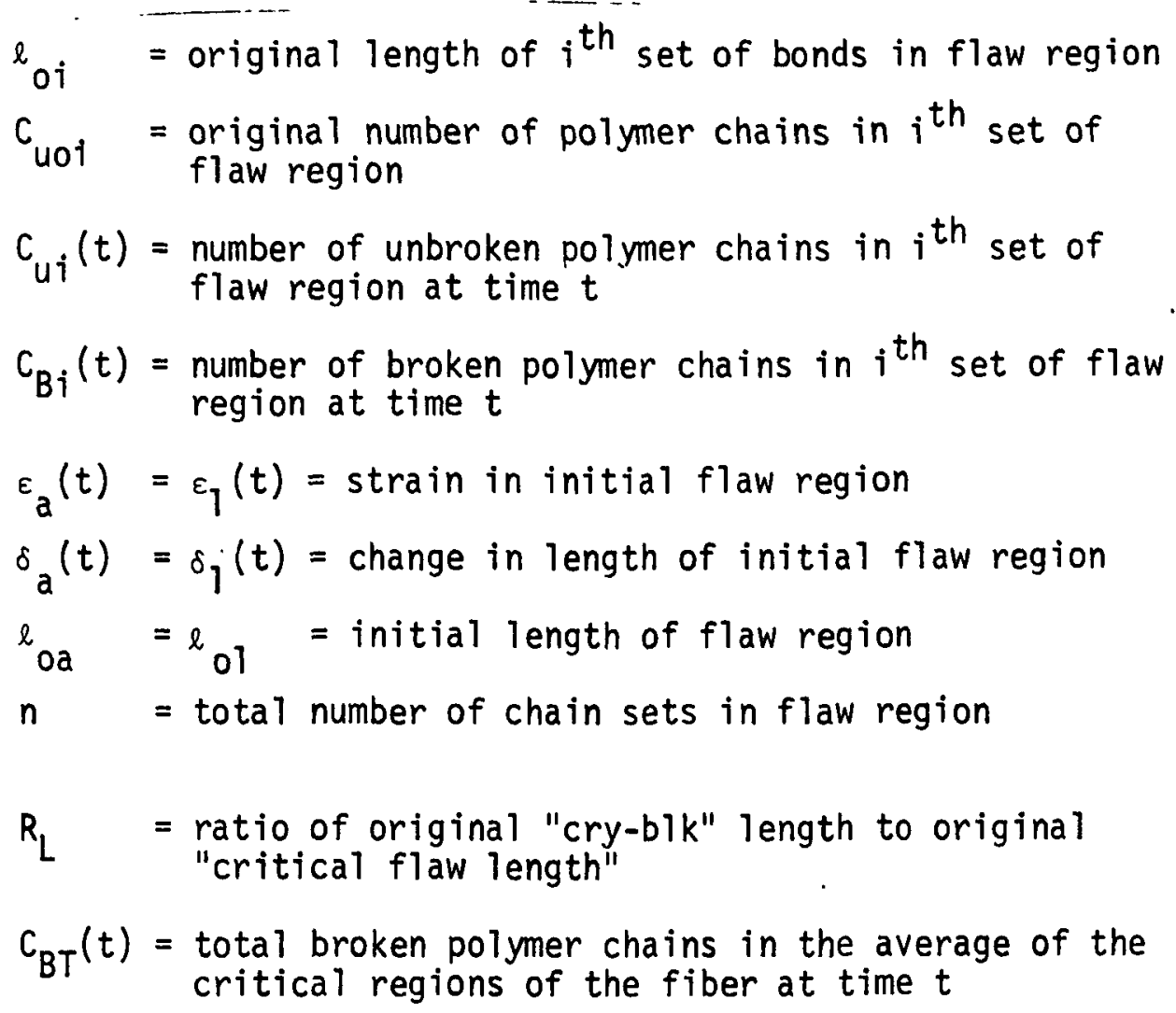

\section{Applied Macroscopic Stress}

Given a macroscopically applied load (or stress), find the stress and strain on the polymer chains, the number of broken bonds or chain scissions and the macroscopic strain as a function of time. For $M$ uniform average regions:

$$
\sigma_{T}=\frac{F_{T}}{A_{T}}=\frac{M F_{C}}{M A_{C}}=\sigma_{C}
$$

Now assuming chain scission is negligible in the "cry-blk" region:

$$
\sigma_{c}=\frac{F_{c}}{A_{c}}=\frac{F_{c}}{C_{0} A_{b}}
$$

a] so

$$
\varepsilon_{c}=\frac{{ }^{\delta} c}{L_{o c}}
$$

Assuming Hooke's law is applicable on an atomic scale and that the average modulus of elasticity in the "cry-blk" region is approximately 
the same as the modulus of elasticity of a single polymer chain, then:

$$
\sigma_{c}=E_{b} \varepsilon_{c}
$$

Making a force balance between the "cry-blk" and "critical flaw" region we have:

$$
F_{c}=\sum_{i=1}^{j} F_{a i}
$$

where $j$ represents the longest taut set of polymer chains in the flaw region at time t. All longer sets of polymers chains i.e. $j<i \leq n$ are assumed to have zero load at this instant. Now, at any given applied stress the chains that are stressed in the flaw region must have the same length,

$$
\ell_{01}+\delta_{1}=\ell_{02}+\delta_{2}=\ldots .=\ell_{0 i}+\delta_{i} \text { for } 1 \leq i \leq j
$$

Solving equation 10 for the strain in the $i^{\text {th }}$ element in terms of the $j^{\text {th }}$ element:

$$
\varepsilon_{i}=\left\{\frac{\ell_{0 j}}{l_{0 i}}\left(1+\varepsilon_{j}\right)\right\}-1
$$

Note that $\varepsilon_{i}, \sigma_{i}$ and $\varepsilon_{j}, \sigma_{j}$ are also the strains and the stresses on the individual bonds in each set respectively if we assume that each set is uniforml:" extended and loaded. Using Hooke's law again we obtain:

$$
\sigma_{i}=\frac{F_{a i}}{A_{a i}}=\frac{F_{a i}}{C_{u i} A_{b}}=E_{b} \varepsilon_{i}
$$

or

$$
F_{a i}=C_{u i} \dot{A}_{b} \ddot{E}_{b} \varepsilon_{i}
$$

Substituting Equation 13 into Equation 9 we get:

$$
F_{c}=A_{b} E_{b} \sum_{j=1}^{j} C_{u i} \varepsilon_{i}
$$


But: $\quad F_{C}=C_{0} A_{b} \sigma_{T}$

Using equations 14 and 15 we now have obtained a relationship

between the applied macroscopic stress and the microscopic strain on each polymer chain in the flaw region:

$$
\sigma_{T}=\frac{E_{b}}{C_{0}} \sum_{i=1}^{j} C_{u i} \varepsilon_{i}
$$

Note that the area of a single polymer chain has cancelled from the equations. Combining equation 11 with equation 16, it is then possible, with some manipulation, to solve for the strain in the $j^{\text {th }}$ element $i . e$. the longest taut set of polymer chains in the flaw region, in terms of the applied macroscopic stress and the number of unbroken polymer chains in the flaw region at any given instant of time.

$$
\varepsilon_{j}=\frac{\frac{c_{0}}{E_{b} C_{u j}} \sigma_{T}-\sum_{i=1}^{j} \frac{C_{u i}}{C_{u j}}\left(\frac{\ell_{0 j}}{\ell_{0 i}}-1\right)}{\sum_{i=1}^{j} \frac{C_{u i} \ell_{0 j}}{C_{u j} \ell_{0 i}}}
$$

The key to the solution of equation 17, i.e. determining which of the flaw sets $1 \leq i \leq n$ is the longest taut set ( $j^{\text {th }}$ set), is obtained by noting that the solution is that value of $j$ which results in the minimum positive value of $\varepsilon_{j}$. Once $\varepsilon_{j}$ is obtained, equations 11 and 12 can be solved for the strain and stress in each polymer chain in all the flaw sets. Compiling these values with the stress and strain in the "cry-blk". regions, obtained from equations 5 and 8 , all the microscopic stress and strain values are then known for the average of all critical regions of the fiber. The macroscopic strain $\varepsilon_{T}$ is: 


$$
\varepsilon_{T}=\frac{\delta_{T}}{L_{\text {oT }}}=\frac{M\left(\delta_{c}+\delta_{a}\right)}{M\left(L_{o c}+l_{o a}\right)} \frac{\delta_{c}+\delta_{1}}{L_{o c}+l_{0.1}}
$$

or in slightly different form:

$$
\varepsilon_{T}=\frac{\varepsilon_{c} \frac{L_{O C}}{c \ell_{01}}+\varepsilon 1}{1+\frac{L_{o C}}{\ell_{o l}}}=\frac{\varepsilon_{C} R_{L}+\varepsilon \frac{1}{1+R_{L}}}{1}
$$

Note again that the key equations, $5,8,11,12,17$, and 19 contain only ratio values of the idealized geometry and that the bond area value $A_{b}$ does not appear. This is both a desirable and undersirable consequence. Desirable from the position that no absolute microscopic values of number of polymer chains or initial lengths in the "cry-blk" and flaw regions are necessary for a solution to the problem. (As will be discussed later, absolute values are not known but some limiting values do exist). Undesirable from the viewpoint that no absolute values can then be obtained from the solution. However, ratio values can be obtained and will give much insight into the fracture properties of the polymer micro-structure.

The next step in formulating the mathematical model is to couple the geometric relationships to the proposed failure criterion for chain scission, that is, to reaction rate theory as proposed in Equation 4. In view of the idealized model where chain scission is assumed to occur only in the "critical flaw" regions and these regions consist of sets of polymer chains of various lengths according to some prescribed distribur tion an equation like equation 4 must exist for each set of equally stressed polymer chains in the "critical flaw" region, i.e.

$$
\frac{d C_{u i}}{d t}=-K_{i} C_{u i} \quad \text { for } i=1, \ldots n
$$

where 


$$
k_{i}=w_{0} \exp \left[-\left(U_{0}-\gamma \sigma_{i}\right) / k T\right]
$$

For constant temperature these equations reduce to:

$$
\frac{d C_{u i}}{d t}=-\left[A \exp B \sigma_{i}\right] C_{u i} . \quad i=1, \ldots, n
$$

where

$$
\begin{aligned}
& A=w_{0} \exp \left(-U_{0} / k T\right) \\
& B=r / k T
\end{aligned}
$$

combining equation 11 and 12 we obtain an expression for the stress $\sigma_{i}$ in the $i^{\text {th }}$ set of the flaw region in term of the strain in the $j^{\text {th }}$ (longest taut) set

( $\sigma_{i}$ for $j<i \leq n$ is assumed to be zero)

$$
\sigma_{i}=E_{b}\left[\frac{\ell_{0 j}}{\ell_{0 i}}\left(1+\varepsilon_{j}\right)-1\right] \text { for } i=1, \ldots, j
$$

The governing set of equations for chain scission is then given by combining equation 17 with equation 22 and substituting the result into equation 21. For the stressed chains $\left(\sigma_{i} \neq 0\right)$ these become:

$$
\frac{d C_{u i}}{d t}=-C_{u i} A \exp \left\{B E_{b}\left[\frac{\ell_{0 j}}{\ell_{0 i}}\left(1+\frac{\frac{C_{0}}{E_{b} C_{u j}} \sigma_{T}-\sum_{j=1}^{j} \frac{C_{u i}}{C_{u j}}\left(\frac{l_{0 j}}{\ell_{0 i}}-1\right)}{\sum_{i=1}^{\frac{C_{u i}}{C_{u j}}} \frac{l_{0 j}}{\ell_{0 i}}}-1\right]\right\}\right.
$$

for the unstressed chains $\left(\sigma_{i}=0\right)$ the equations are:

$$
\frac{d C_{u i}}{d t}=-C_{u i} A \quad \text { for } j<i \leq n
$$

Once these equations are solved for the number of unbroken chains in the $i^{\text {th }}$ set, the number of broken chains or chain scissions for that set is given by: 


$$
C_{B i}=C_{u o i}-C_{u i}
$$

and the total chain scission in the average critical region of the fiber is:

$$
C_{B T}=\sum_{i=1}^{n} C_{B i}
$$

Examining equation 23 we observe that this is a set of simultaneous lst order non-linear differential equations. Considerable thought has been devoted, to methods by which an analytical solution might be obtained to this set and while this possibility is not to be ruled out, the author elected to solve the problem numerically with the aid of the Univac 1108 computer.

Given a macroscopically applied extension (or strain) find the stress and strain on each polymer chain, the number of broken bonds or chain scissions and the macroscopic stress as a function of time.

The solution to this problem is very similar to that of the applied macroscopic stress. Noting in the previous derivation that the relationship obtained in. equation 16 between the applied macroscopic stress and the microscopic strain on each polymer chain in the flaw region can be written in terms of strain in the "cry-blk" region using the relationship:

$$
\sigma_{T}=\sigma_{C}=E_{b} \varepsilon_{C}
$$

we have

$$
\varepsilon_{c}=\frac{1}{c_{0}} \sum_{i=1}^{j} c_{u j} \varepsilon_{i}
$$

Equation 28 can be written in terms of the strain in the $j^{\text {th }}$ set using 
equation 11:

$$
\varepsilon_{c}=\frac{1}{c_{0}} \sum_{i=1}^{j} c_{u i}\left\{\frac{l_{0 j}}{l_{0 i}}\left(1+\varepsilon_{j}\right)-1\right\}
$$

Also, equation 19 for the macroscopic strain can be written in terms of the "cry-blk" strain $\varepsilon_{c}$ and the strain in the $j^{\text {th }}$ set of the "critical flaw" region, i.e.

$$
\varepsilon_{T}=\frac{\varepsilon_{c} R_{L}+\frac{\ell_{0 j}}{\ell_{01}}\left(1+\varepsilon_{j}\right)-1}{1+\tilde{R}_{L}}
$$

Equation 29 is then substituted into equation 30 and, after some manipulation, the strain in the $j^{\text {th }}$ set (longest taut set) can be written in terms of the applied macroscopic strain and the number of unbroken polymer chains in the flaw region at any given instant of time.

$$
\varepsilon_{j}=\frac{\left.\left(R_{L}+1\right) \varepsilon_{T}+1-\frac{\ell_{0 j}}{\ell_{01}}-\frac{R_{L}}{C_{0}} \sum_{i=1}^{j} C_{u i\left(\frac{\ell_{0 j}}{\ell_{0 j}}-1\right.}\right)}{\frac{\ell}{0 j}_{\frac{l}{\ell_{01}}}+\frac{R_{L}}{C_{0}} \sum_{j=1}^{j} C_{u i} \frac{{ }_{0 j}}{\ell_{0 i}}}
$$

The derivation from this point is now the same as outlined for the applied macroscopic stress.

The governing equations are then equations 31, 11 and 12 for the polymer chains in the flaw region; equations 29 and 27 for the strain and stress in the "cry-blk" regions; and equation 27 for the macroscopic stress. Chain scission is determined from the rate equation lequation 21) where the stress $\sigma_{i}$ is given by the conditions specified by equation 22, using Equation 31 for the strain in the $j^{\text {th }}$ set in terms of the applied macroscopic strain. Once these equations are solved the total chain scission in the average of the critical regions of the fiber is determined by equation 25 and 26 . 
The basic assumptions in the idealized mathematical model of the polymer fiber can be summarized as:

1. The shear mode of failure i.e. chain slippage or flow due to secondary bond failure in the average of the critical regions is negligible compared to backbone chain scission.

2. Chain scission in the "crystalline block" region is negligible.

3. Hooke's law is applicable on an atomic scale.

4. A chain assumes no load-carrying ability until the strain in the flaw region is such that the chain will be taut and after it is taut, it is assumed to act elastically.

5. Chain scission is predicted by kinetic reaction rate theory. The numerical approach to the solution of the above model will be briefly outlined with the aid of Computer Flow Diagrams 1 and 2 . The first step in the solution is to prescribe some type of distribution of tie chains in the "critical flaw" region. Experimental results from step strain tests indicate that the distribution might best be approximated with a Gaussian or normal distribution criterion. Flow Diagram 1 outlines how the tie chains are apportioned among the sets according to an experimentally determined standard deviation. The distribution in effective chain length may be continuous in the actual polymer fiber, however, for numerical purposes we assume this continuous distribution can be replaced by sets of chains.

once the distribution of chain number and length in each set of the flaw'region is determined the governing equations for an applied 
macroscopic stress (or strain) are solved as outlined in computer Flow Diagram 2. For example, if the input load is the macroscopic stress, the strain (or elongation) of each chain in a given flaw set is the same but differs, of course, from set to set. "Slack" sets of chains are assumed to experience no strain until the strain of the region exceeds the percentage slack of the given set. In reality slack chains can exert entropic forces. A further refinement of the model could include these forces using rubber elasticity theory and further coment on this will be made later. Therefore, for a given $\sigma_{T}$ Equation 17 is solved by successive iteration to determine the strain in the $j^{\text {th }}$ set of elements. Using this value, Equations 11 and 12 are solved for the strain and stress in each set, $i=1, j$. (The stress in sets $j<i \leq n$ is zero).

For a given temperature these values are substituted into Equation 21 and this simultaneous set of equations numerically integrated to give the change in the number of unbroken bonds in the sets in the flaw region for a given time interval. The numerical integration could be accomplished with the simple one step approximation of Euler's method or by the more sophisticated 4th Order Runge-Kutte approach. In practice, it was found that Euler's method for the time interval (one second) and number of sets chosen (101 sets) was sufficiently accurate and therefore, to save computer time it was used almost exclusively. The total chain scission, "cry-b1k" stress and strain and macroscopic strain were the calculated using Equations 26, 5, 8, and 19 respectively. 
FLOW DIAGRAM

DISTRIBUTION COMPUTER PROGRAM

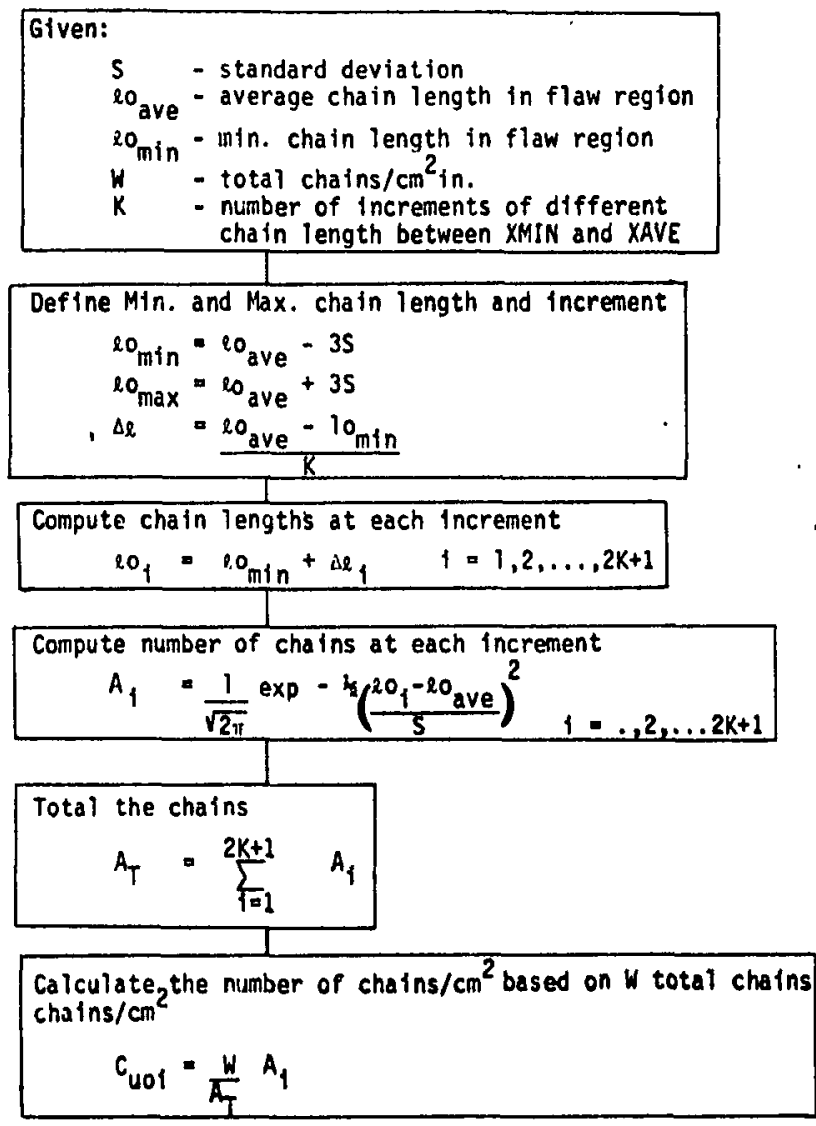




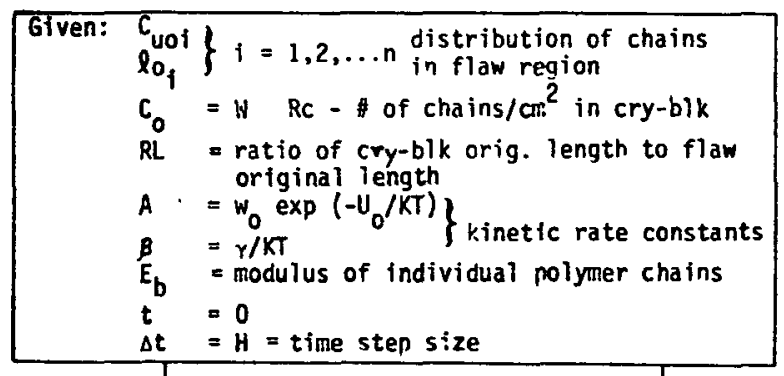

$\Delta t=H=$ time step size

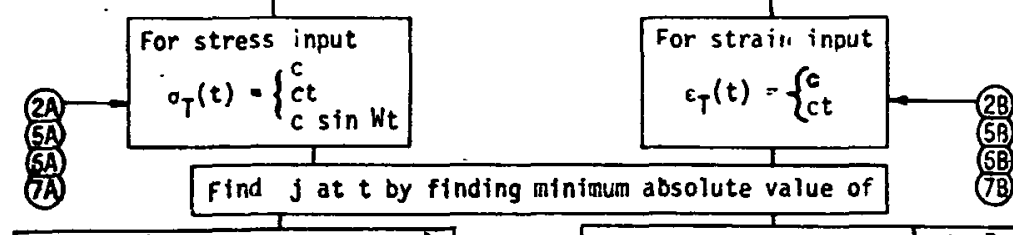

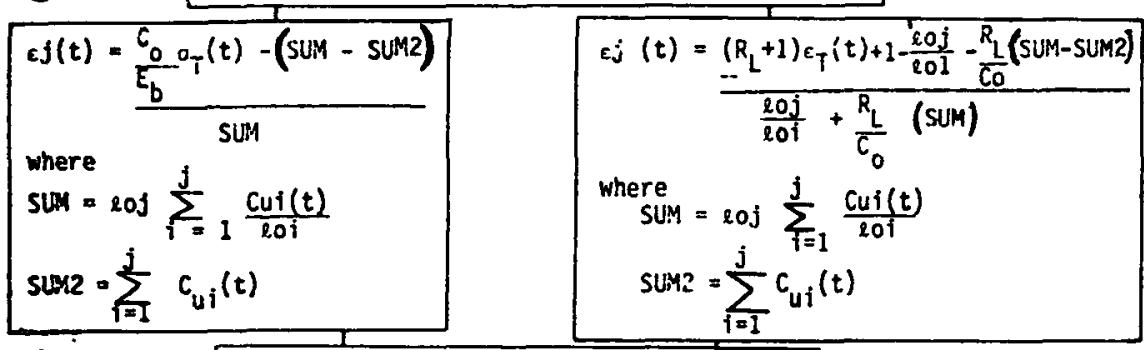

$=$ anow $j$ ivalue sand $e j(t)$ are knowri, calculate

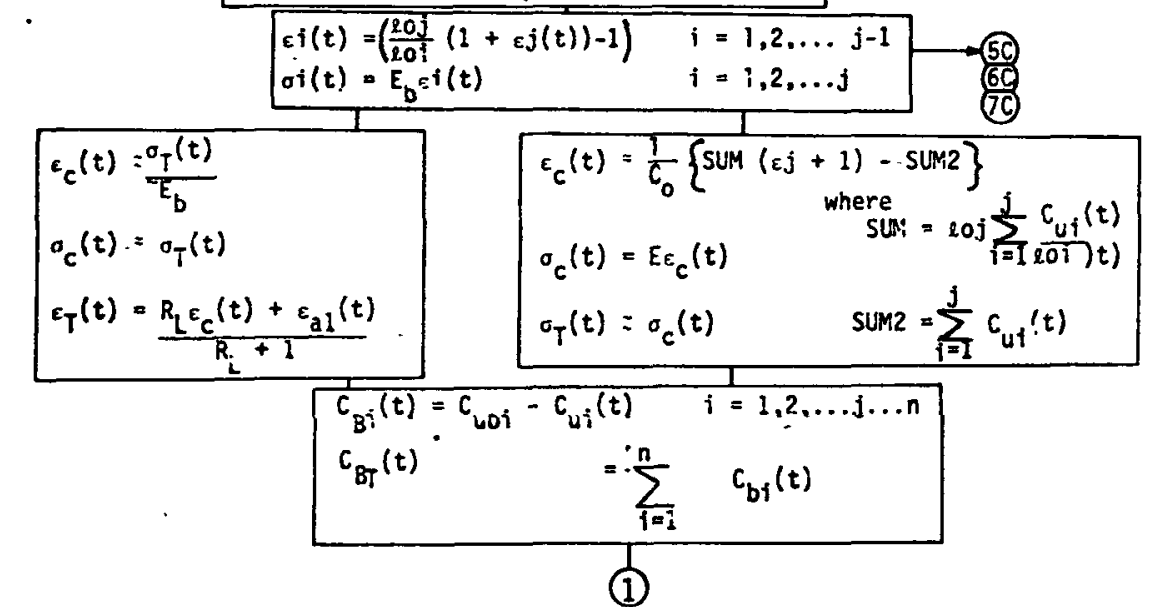

FLOW DIAGRAM $\$ 2$ (cont)

(1)

Now calculate the rate at which

bonds are being broken at time $t$

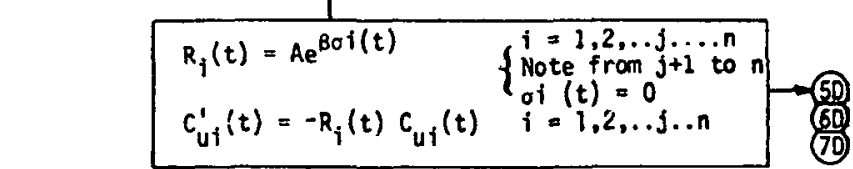

Calculate the new set of unbroken

bonds $C_{u f}(t) ! s$ at $t+\Delta t$ using either
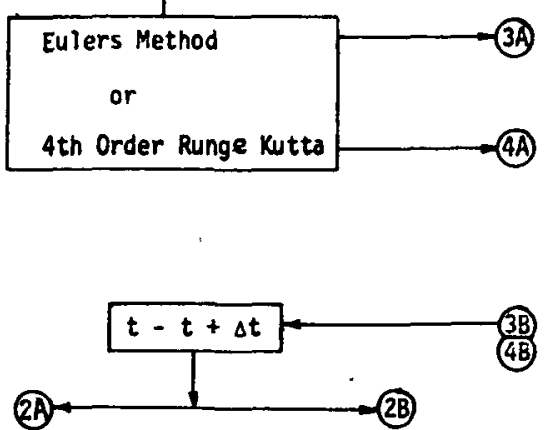
FLOW DIAGRAM 2 (cont)

(3A) Euler's Method

(BB). $-C_{u i}(t+\Delta t)=C_{u i}(t)+H C_{u i}(t) \quad i-1,2, \ldots j \ldots n$

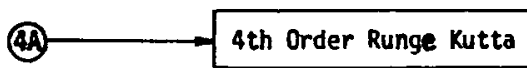

$$
\begin{aligned}
& t_{1}=t \\
& c_{u i}\left(t_{1}\right)=c_{u i}(t) \quad 1-1,2, \ldots . . . n
\end{aligned}
$$

Calculate $K_{0}$

$$
K_{0 i}(t)=H C_{u i}^{\prime}(t) i-1,2, \ldots . \ldots n
$$
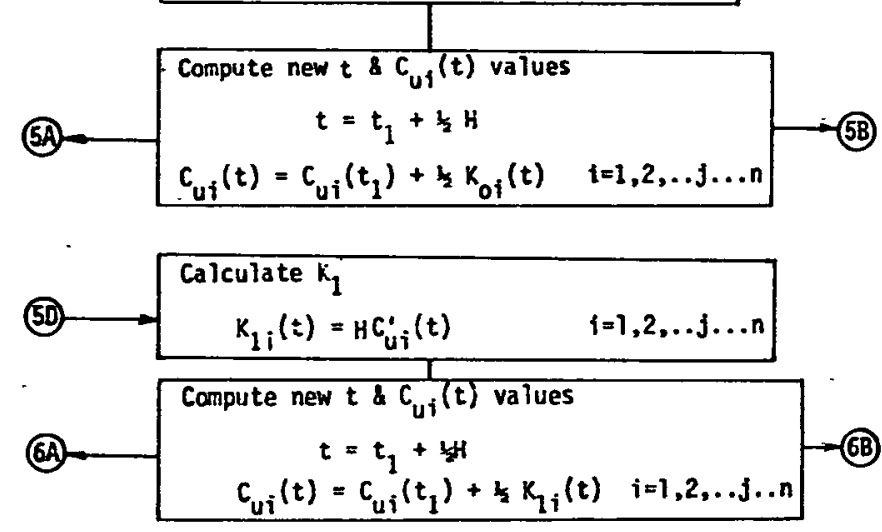

Calculate $K_{2}$

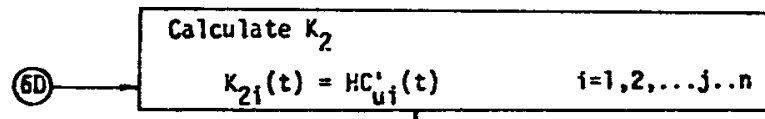

Compute new $t \& C_{u i}(t)$ values

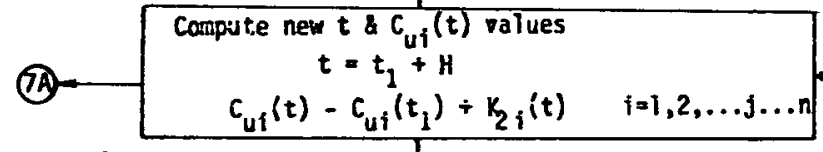

FLOW DIAGRAM \#2 (cont)

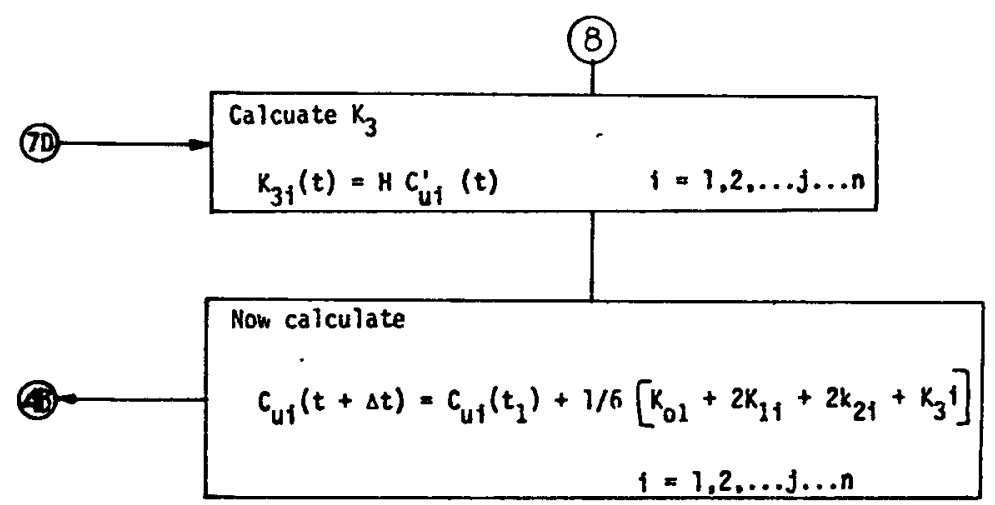

un 
The input parameters necessary for a theoretical solution using the mathematical model of the polymer fiber are:

1. $S=$ the standard deviation value of the distribution of chains in the flaw regions

2. $R C=$ the ratio of the number of polymer chains in the "cry-blk" region to the number of tie chains in the flaw region

3. $\mathrm{RL}=$ the ratio of the original length of the "cry-blk" region to the original length of the flaw region.

4. $E_{b}=$ the modulus of elasticity of a single polymer chain

5. $w_{0}=$ the collision parameter

6. $U_{0}=$ the activation energy

7. $\gamma=$ the activation volume

8. $\sigma_{T}$ or $\varepsilon_{T}=$ the applied macroscopic stress (or strain) as a function of time

9. $T$ = the absolute temperature

Some additional arbitrary parameters included in the program are necessary for numerical computations and some are included to allow facilities for obtaining absolute values of the micro-structure elements. These are:

1. $K=$ the number of sets of polymer chains in the flaw region

2. $H=$ the time increment for the numerical integration

3. XMIN = the minimum length of chain set in the flaw region distribution at time zero

4. $W=$ the total chains $/ \mathrm{cm}^{2}$ in the flaw region at time zero

The values of $K$ and $H$ most used were 101 sets and one second respectively, however, variations of these values were checked to be 
certain that the computer solutions were relatively insensitive to the stable values chosen. Theoretically a Gaussian distribution has no cut off point, however 99.74 per cent of the distribution lies within $\pm 3 S$ of the mean value. This $\pm 3 S$ value was used as the XMIN point, however, the XMIN length value chosen at this point was purely arbitrary. The total number of chains $/ \mathrm{cm}^{2}$ in the flaw region was also an arbitrary choice. Even so, in order to obtain chain scission results that could be compared with experimental observations, an "in-the-bal1-park" number was necessary. The choice of this value and the method of obtaining the basic input parameters for the model will be discussed in depth in the following sections.

\subsection{MODEL PARAMETERS AND ROOM TEMPERATURE TESTS}

At the outset, it should be stated that absolute values of the atomic parameters required as input values for the idealized mathematical model are not known. At best, there exists only exerpimentally based limiting type values or calculated values based on theoretical premises. As a result of this fact, one of the main objectives of this study was not only to use the model to verify the theoretical assumptions, but to also use is as a tool for refining or establishing the necessary input parameters. The following is a brief summary of information related to the input parameters values.

1. The Distribution Parameter "S"

As pointed out in section 2.3, the key to many of the experimentally observed properties of these highly-oriented fibers seemed to indicate a distribution of stresses in the polymer microstructure. Theoretical calculations of the strength of a single polymer chain result in tensile 
strength values of approximately $10,000 \mathrm{ksi}$. However, tensile strength of most of the highly-oriented polymer fibers rarely exceeds $150 \mathrm{ksi}$. This large difference has been explained as a consequence of the inhomogeneity of the micromorphology in the fiber structure. Chain ends, dislocations, disorder, disorientation, chain folds, and number and length of tie molecules have all been suggested as elements that prevent a uniform stress distribution in the fiber and result in regions of high stress concentration which in turn lower the maximum attainable tensile strength $(9,13,23,46,47)$. With the advent of $E P R$, it was hoped that a more qualitative analysis of the microstructure could be assessed from experimentally observed chain scission and shed additional "light" on the quantitative proposals. The failure of the Zhurkov theory, where a uniform stress distribution was assumed, to predict chain scission as experimentally observed in creep tests gave additional credibility to the idea of flaw regions existing, in part, of tie molecules distributed in number according to their effective length $(9,32,13,33,34-36)$. Even so, it was not until this study that experimental evidence for such an "effective or apparent distribution" was observed and qualitately analyzed. The idea of characterizing the histograms from EPR step strain tests in relationship to the tie chains in the critical flaw regions turned out to be the key results in the success of this study. It was from the results of figures 17 and 18 that the distribution was chosen to be characterized by the one standard deviation value of a normal or Gaussian distribution. Further step strain tests at different tempatures and on different nylon fiber samples were undertaken to observe 
Page Intentionally Left Blank 
the influence of the effective distribution on the ultimate strength of the fibers tested. These tests included: two sets of nylon 6 samples (nylon6 \#1 and nylon $6 \# 2$ ) at temperatures of $-25^{\circ} \mathrm{C}$, room temperature $\left(+22^{\circ} \mathrm{C}\right),+50^{\circ} \mathrm{C}$; and $-25^{\circ} \mathrm{C}$, room temperature, $+50^{\circ} \mathrm{C},+75^{\circ} \mathrm{C}$, $+100^{\circ} \mathrm{C}$ respectively; a room temperature test of a modified slack annealed sample* of nylon 6 \#2 and a nylon 66 sample at room temperature. Figures $21,22,23$, and 24 show the results of these tests plotted as histograms.

The effect of the width of the distribution on the maximum stress in these polymer fibers can be observed in Figure 25 . The interesting result from this plot is that a direct correlation appears to exist between the width of the distribution and the ultimate strength independent of the type of nylon fiber. This will be discussed in depth later. Therefore, as the width of the distribution decreases, the maximum strength of the fiber increases.

One can gain some insight of this effect by considering the extremes. If the chains were all equally stressed, corresponding to a standard deviation of zero, the strength of the fiber would be the "strength" of each chain multiplied by the number of chains across the critical cross-section of the sample. Alternately, one might envision such a wide distribution in chains that one chain is stressed and ruptured at a time. In this case the strength of the fiber reduces to the "strength" of the individual chains although the energy dissipated tension at $200^{\circ} \mathrm{C}$ for two minutes and slowly cooled to room temperature before step strain test. 


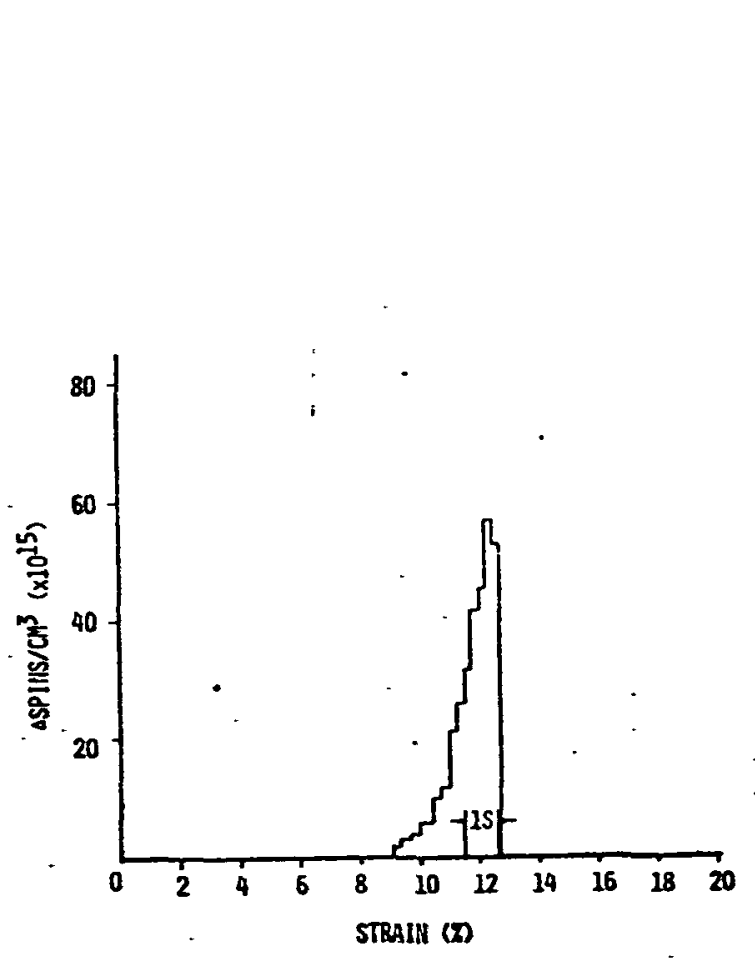

a. $-25^{\circ} \mathrm{C}$

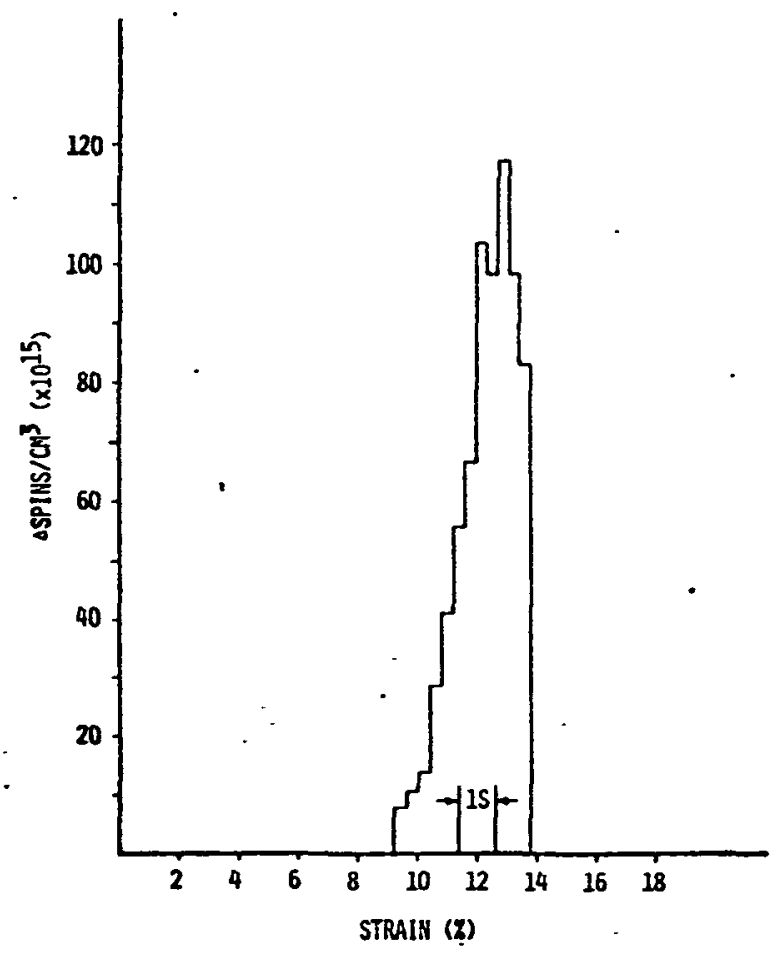

b. Room Temperature

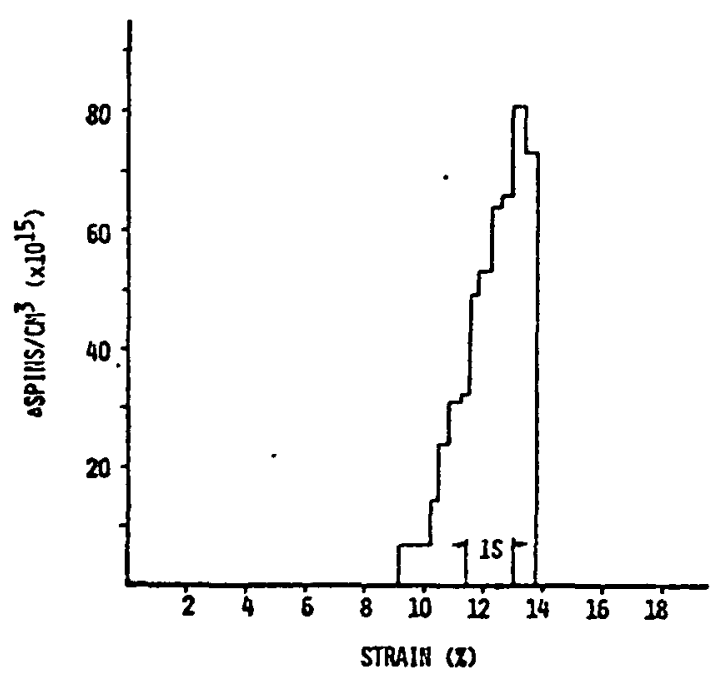

c. $+50^{\circ} \mathrm{C}$

FIGURE 21. Histograms of nylon 6 \#1 samples from step-strain data at various temperatures. 


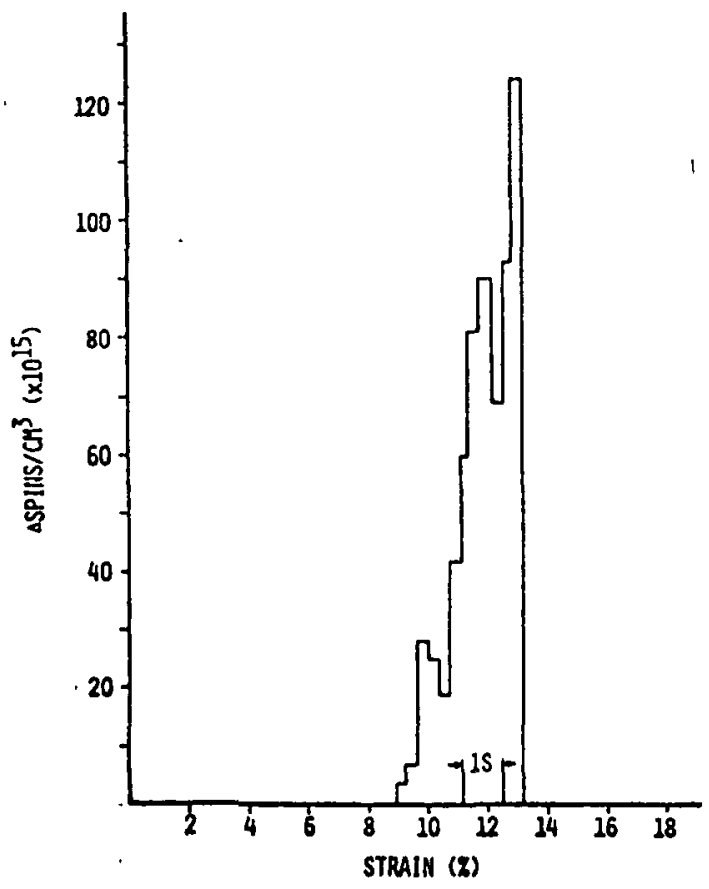

a. $-25^{\circ} \mathrm{C}$.

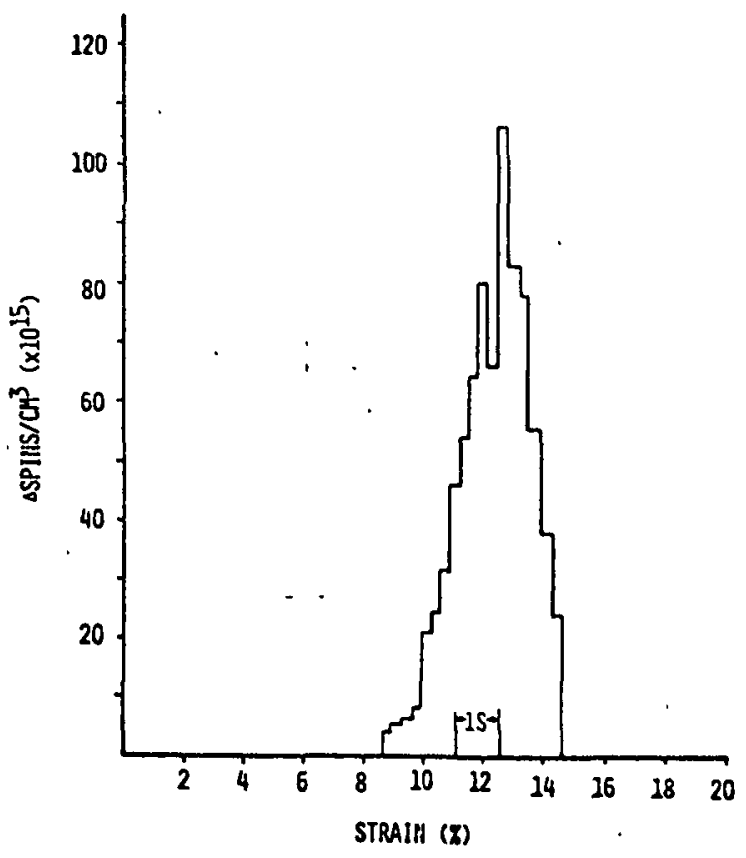

b. Room Temperature

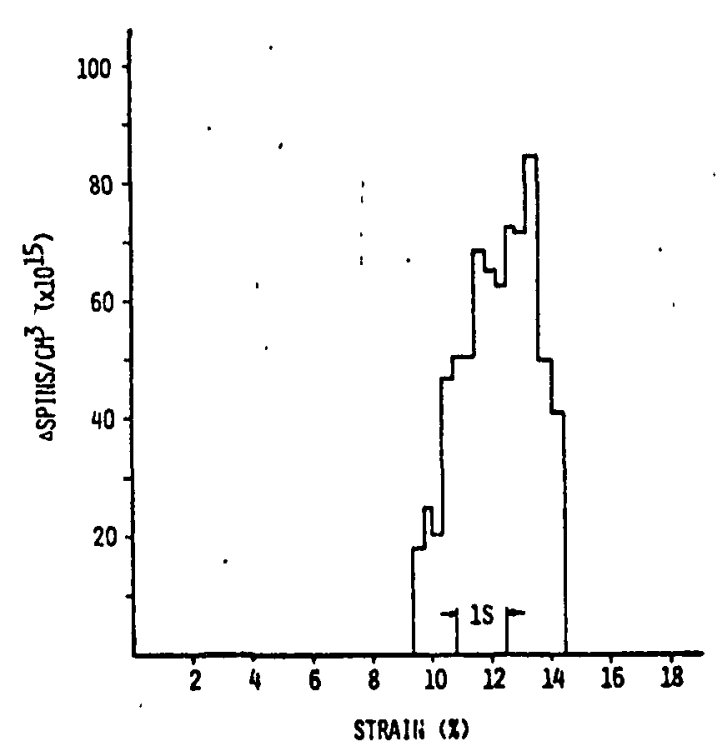

c. $+50^{\circ} \mathrm{C}$

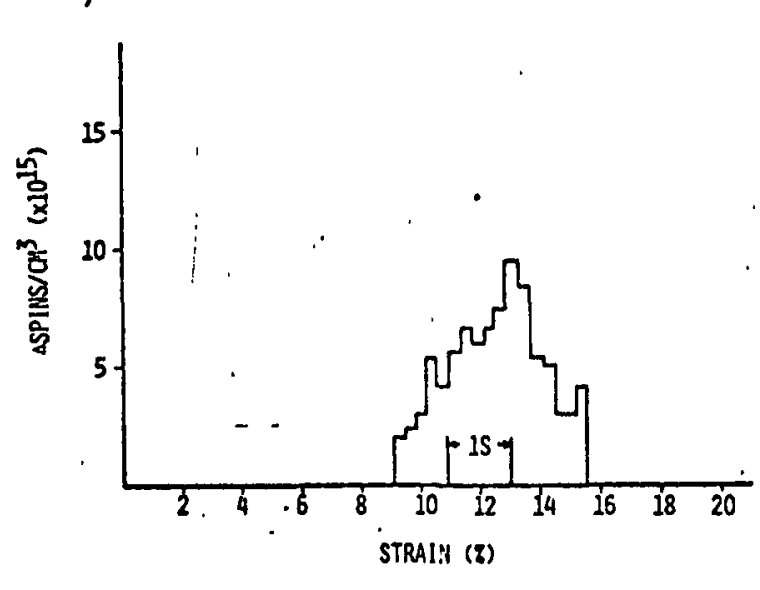

d. $+100^{\circ} \mathrm{C}$

FIGURE 22. Histograms of nylon 6 \#2 samples from step-strain data at various temperatures. 


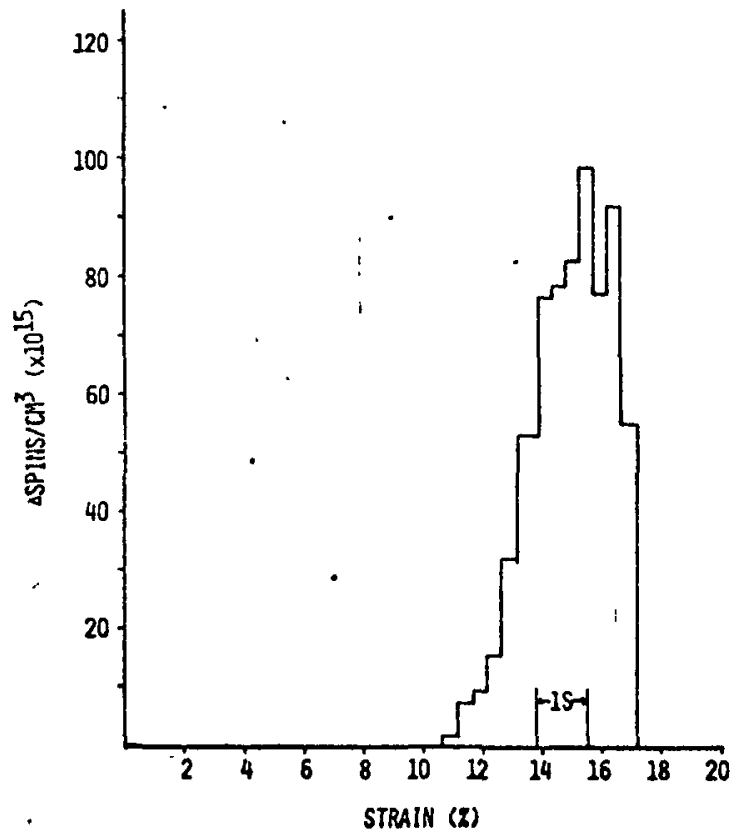

FIGURE 23. Histogram of nylon 6 \#2 annealed sample from stepstrain data at room temperature.

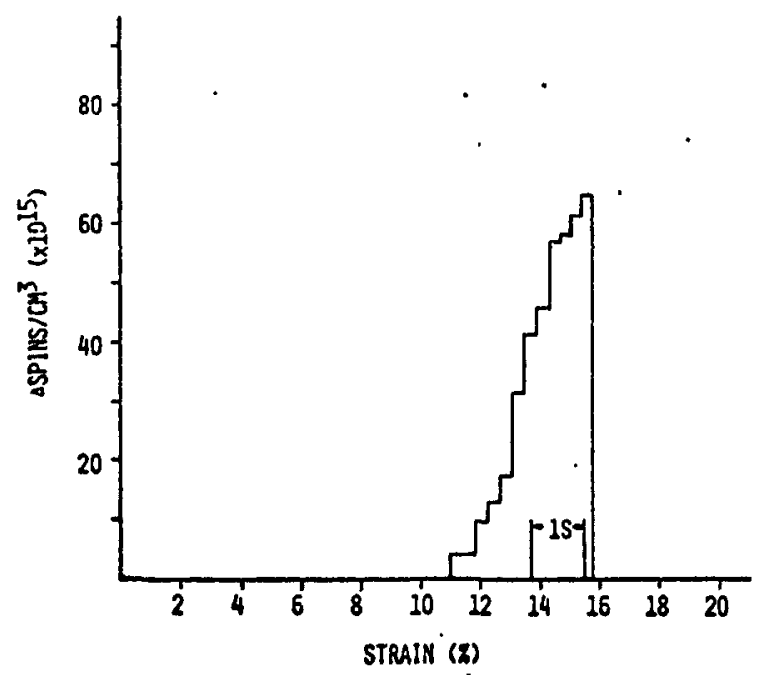

FIGURE 24. Histogram of nylon 66 sample from step-strain data at room temperature. 


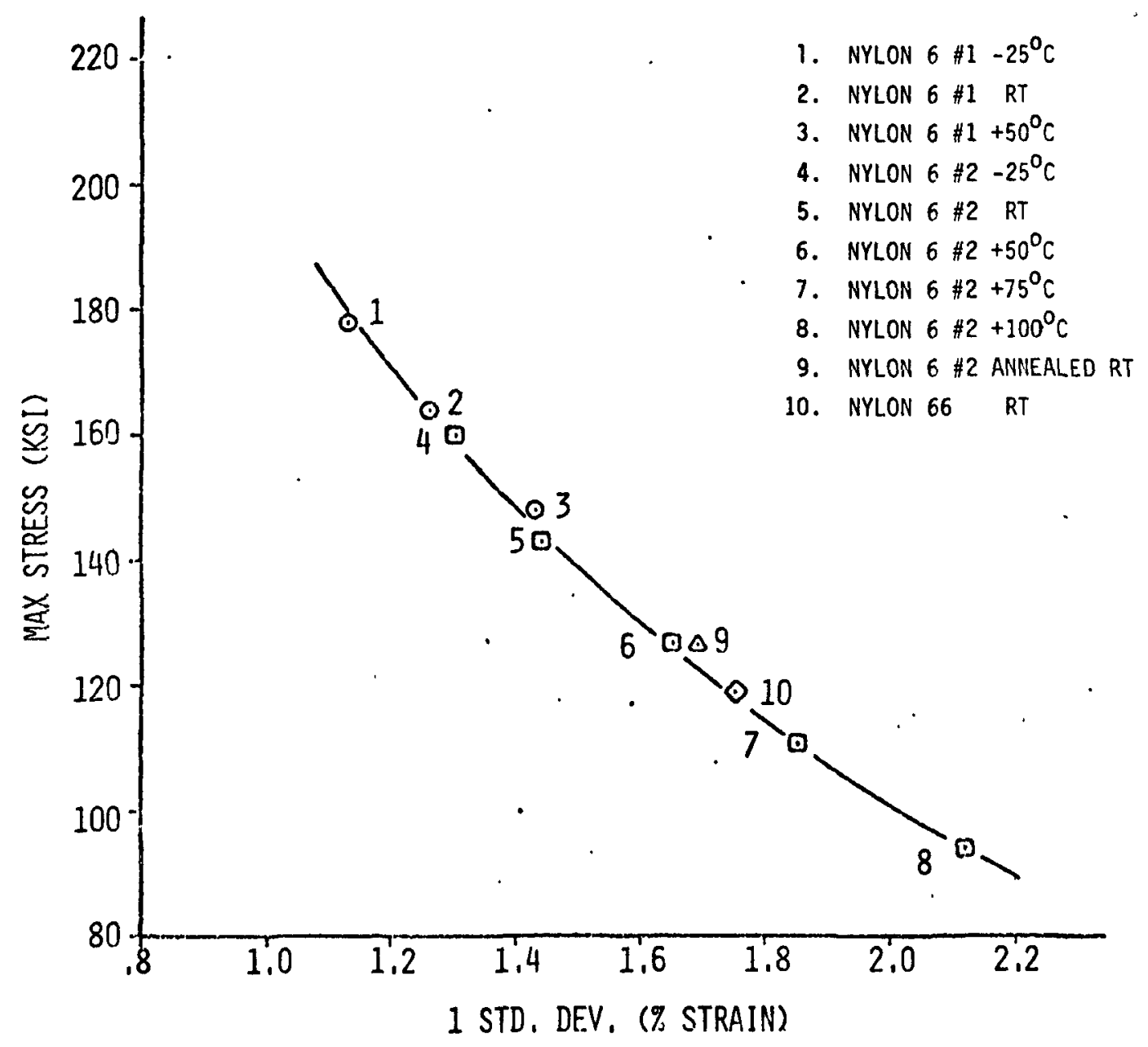

FIGURE 25. Effect of apparent or effective distribution on ultimate strength of nylon fibers 
during fracture and the strain at fracture are both greatly increased. Real fibers behave more nearly like the former than the latter of these extremes but still it is rather amazing the very large differences that small changes in the standard deviation of chain length can have on fiber strength. It should be noted that the term "effective or apparent distribution" has been used for describing the experimental observations. For instance, note the apparent narrowing of the distribution below room temperautre and the broadening above room temperature in Figures 21,22 , and 25 . This change in the apparent or effective distribution with temperature gives important insight into the properties of the microstructure and merits a more detailed discussion. Polymer chains in the flaw region are in varying states of configuration and movement depending on several factors, such as, intermolecular forces, free volume, steric hinderances and thermal environment. The effect of temperature changes on the local surroundings can be thought of as effectively loosening or tightening the structure. Tie chains are undoubtedly not rigidly embedded in the "crystalline block" material and as a result, depending on the temperature or loading rate, some may pul1 out (or partially pull out) rather than rupture. Alternately or in conjunction with this effect, tie chains in kinked, coiled, folded, or other non-extended configurations will most likely exhibit some relaxation, flow, unfolding or unkinking if aided by an increase in temperature. Likewise, with decreasing temperature, chains will tend to break prior to annihilation of kinks, coils, or folds and then the effective length of the chain may be much different than the extended length. Therefore, a decrease temperature or a high loading rate 
should shift part of the chain length distribution to a smaller effective length and a narrower distribution. The opposite effect would be expected for higher temperatures. This effect is verified by the experimental data in Figure 21,22 and 25 . Figure 26 is a schematic presentation of these ideas. The original unstressed tie chains are shown in Figure 26a. At low temperature, some of the chains are "frozen" and have smaller and more uniform effective lengths resulting in a narrow distribution (Figure 26b). As the temperature increases, more unfolding or pulling out takes place resulting in a broader effective distribution as shown in Figure $26 \mathrm{c}$ and $d$.

These essentially reversible effects are not to be confused with irreversible annealing effects which take place rather rapidly above $100^{\circ} \mathrm{C}$. An exhaustive study has just been completed by Joon Park under the direction of W. 0 . Statton on the effects of "stretch" and "slack" annealing on nylon 6 fibers (23). The slack annealed sample of Figure 23 and shown as point 9 in Figure 25 exhibited some important differences to those pointed out above. Besides exhibiting a broader distribution and lower strength at room temperature than the control sample (point 5 in Figure 25), the distribution was shifted along the strain axis to larger strain values. Results from Park (23) indicate that during slack annealing the polymer chains tend to become more disoriented and chain folding occurs which may tend to pull some tie chains into the ordered regions, decreasing the total number and relaxing the stresses in the tie chain regions. Figure 27 is a schematic representation of the type of irreversible effects that "slack" annealing and "stretch" annealing may produce in these regions relative to the control sample. 
ISTS畉产

a. Unstressed control

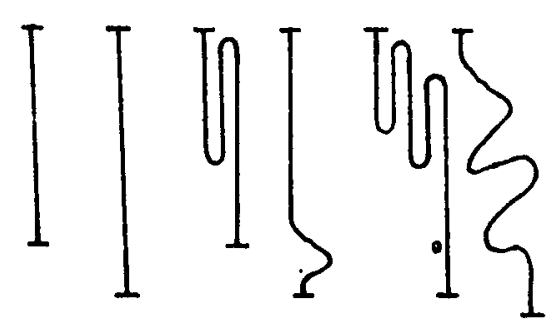

b. Stressed low temperature
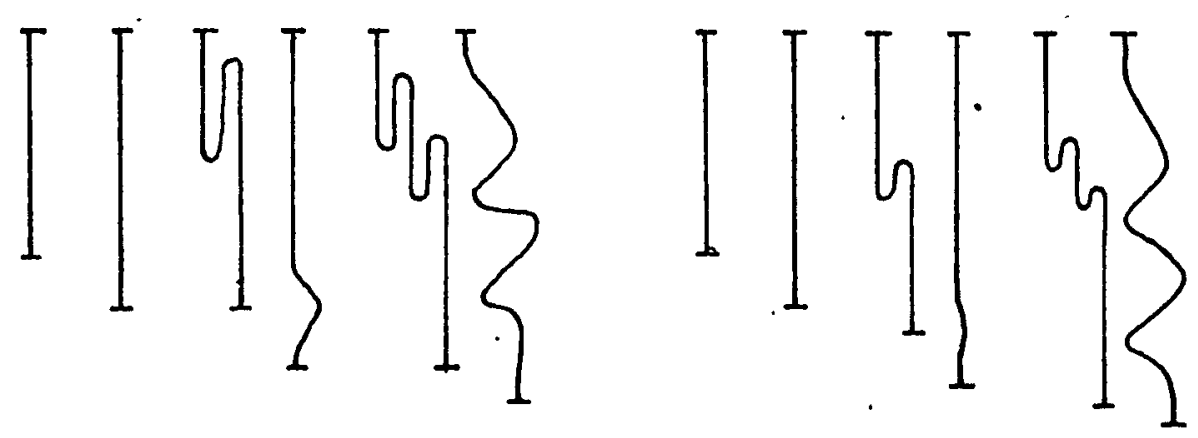

c. Stressed room temperature

d. Stressed high temperature

FIGURE 26. Schematic representation of changes in effective tie-chain distribution with temperature. 


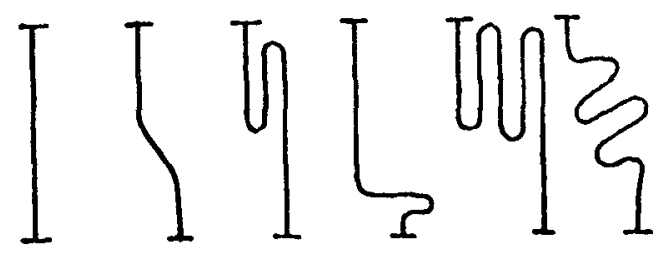

a. Unstressed control

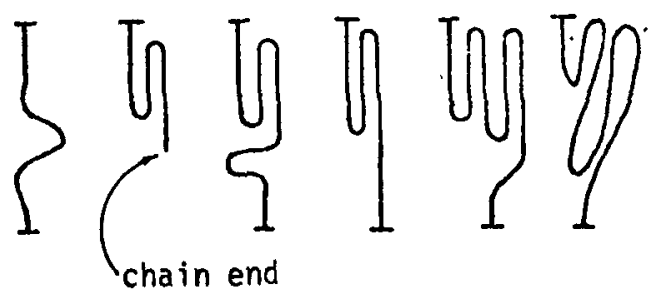

b. Unstressed slack annealed

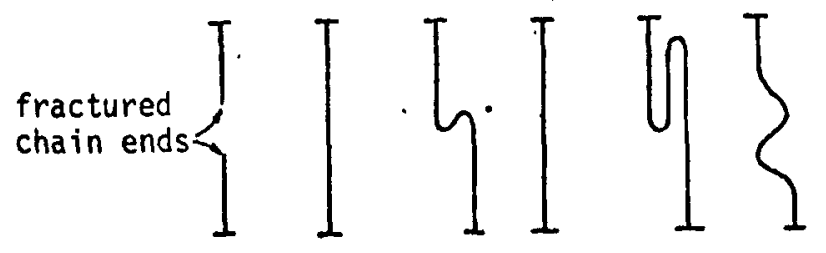

c. Unstressed tension annealed

FIGURE 27. Schematic representation of irreversible type changes created in the tie-chain distribution under slack and tension annealing 
From Figure $27 \mathrm{~b}$, one can visualize a shift along the strain axis due to the overall effective length increase in the tie molecules and a broader distribution. The lower strength may be due to two effects; fewer tie molecules and less uniform effective chain lengths. In general, one would not expect a direct correlation between the "effective distribution" and the maximum strength, independent of the nylon type or treatment unless the total number of tie chains was approximately the same. For example, if sample \#1 has three tie chains of equal length and sample \#2 has six tie chains of equal length, then both have the same distribution, but sample \#2 is twice as strong as sample \#1. From the above reasoning, the results of Figure 25 need to be analyzed in terms of the theory to determine the contribution of each of the above effects to the observed experimental results. This will be discussed next. A1though "stretch" annealed samples were not tested and characterized by these step strain tests, Figure $27 \mathrm{c}$ indicates the effect of this process as explained by Park (23) on the tie molecules. Essentially, some chains may be fractured in the "stretch" annealing process but in general the chains become more oriented thus narrowing the distribution and increasing the strength.. Further work characterizing "stretch" and "slack" annealed distribution properties should be investigated in the near future. Figure 28 shows a plot of distribution width versus temperature for the nylon samples tested. These sets of curves may be thought of as depicting the degree of uniform chain orientation in the critical flaw regions relative to the type of nylon. For instance, at room temperature, nylon 6 \#1 exhibits a narrower distribution (more chains of the same effective length) than does nylon $6 \# 2$ at the same temperature. Likewise, nylon $6 \# 2$ at $-25^{\circ} \mathrm{C}$ 


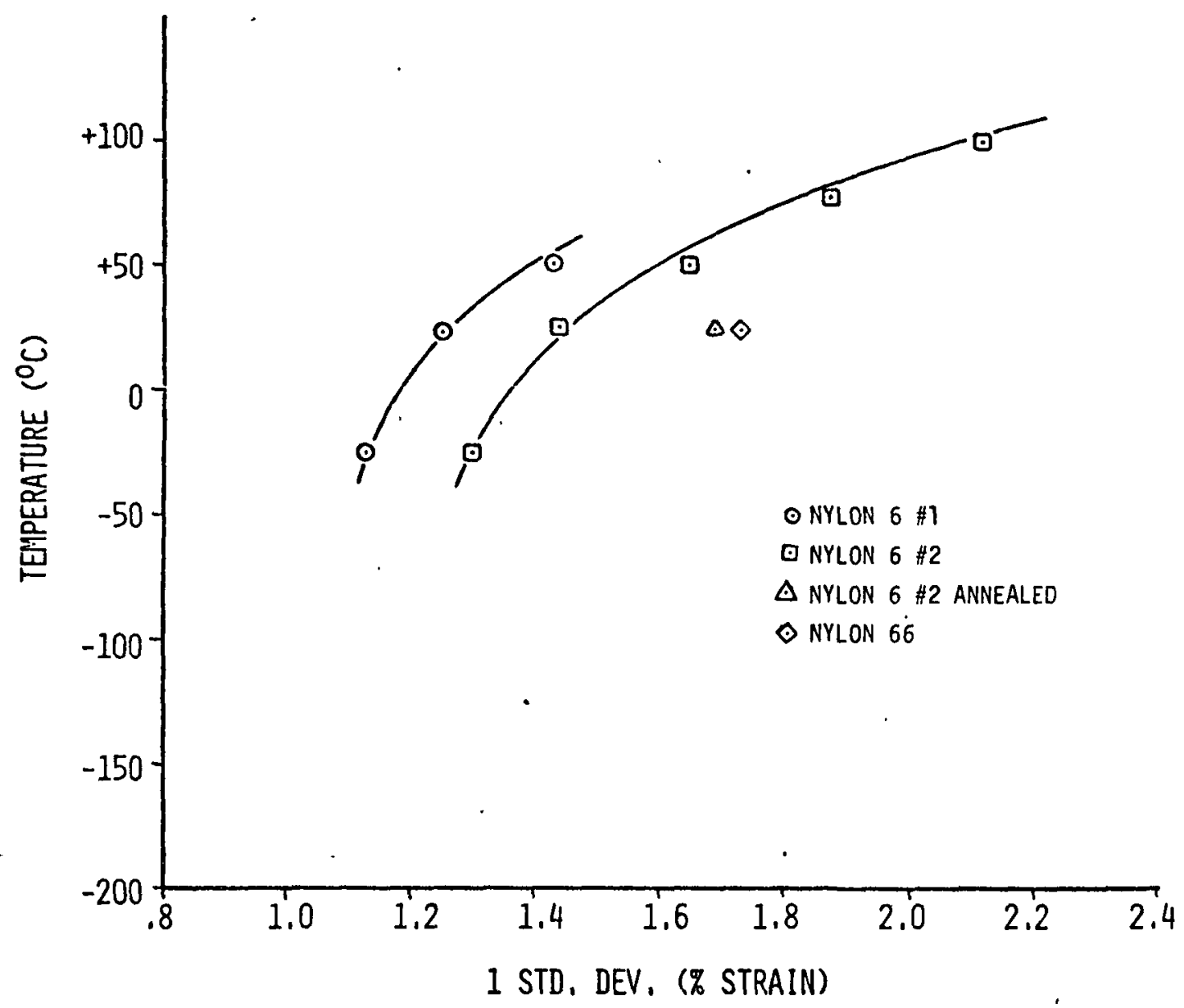

FIGURE 28. Relationship between temperature and effective distribution for various sample materials 
would be expected to exhibit similar properties to nylon 6 \#2 at room temperature as their effective distributions are similar. In general, nylon 6 \#1 would be expected to be more brittle and stronger than nylon 6 \#2 at the same temperatures. Similar shaped curves would be expected for the slack annealed and nylon 66 samples but shifted as shown by the room temperature values.

Analyzing the above results, the importance of the tie chain distribution in the critical flaw regions is readily evident and any model which is proposed to explain the failure mechanism in highlyoriented fibers should include this distribution as a parameter. 2. The Chain Ratio Parameter "RC"

The ratio of the number of chains in the "cry-blk" regions to the number of tie chains in the critical flaw region can be thought of as a stress concentration factor. From polymer single crystal data (48) a single crystal of nylon 6 has the dimensions shown in Figure 29. From this perfect crystal, the effective cross sectional area of a single nylon 6 chain is calculated as $17.7 \mathrm{~A}^{2}$. This results in $5.65 \times 10^{14}$ chains $/ \mathrm{cm}^{2}$, which represents the maximum number of chains that would occur in a square centimeter of polymer cross section. The density of 100 per cent crystalline nylon 6 (calculated from single crystal data) is reported as $1.23 \mathrm{gm} / \mathrm{cm}^{3}$ whereas 100 per cent amorphous (extrapolated from bulk polymer data) is 1 isted as $1.10 \mathrm{gm} / \mathrm{cm}^{3}$ (49). If we considered the flaw regions as 100 per cent amorphous, this would correspond to only a ten per cent reduction in chains per square centimeter compared with the crystalline regions. It is apparently not known if the ordered regions in the highly drawn fibers are really perfect crystals or if the density of the disordered amorphous or flaw 
UNIT CELL of 6 NYLON

$\alpha$ form: $\sigma=9.56 \dot{A}$

$b=17.24 \dot{A} \cdot$ MONOCLINIC

$c=8.01 \AA$

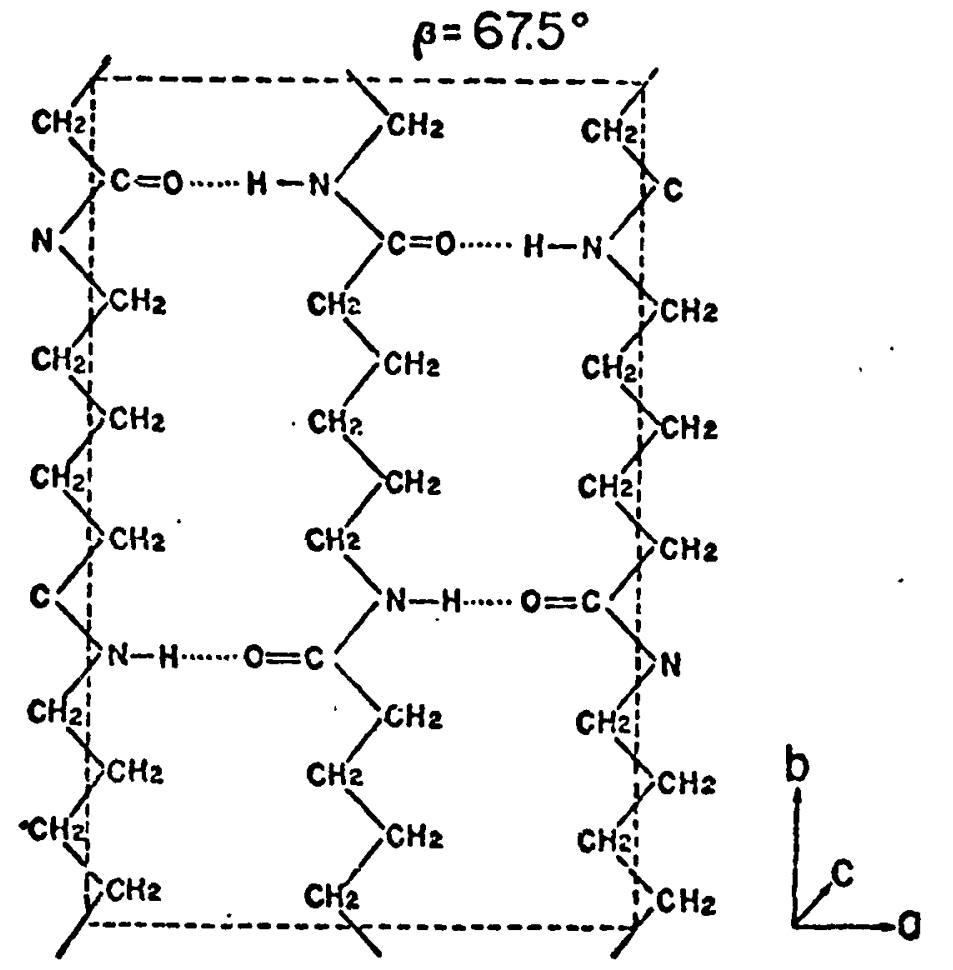

6 NYLON, BASAL PLANE Of UNIT CELL

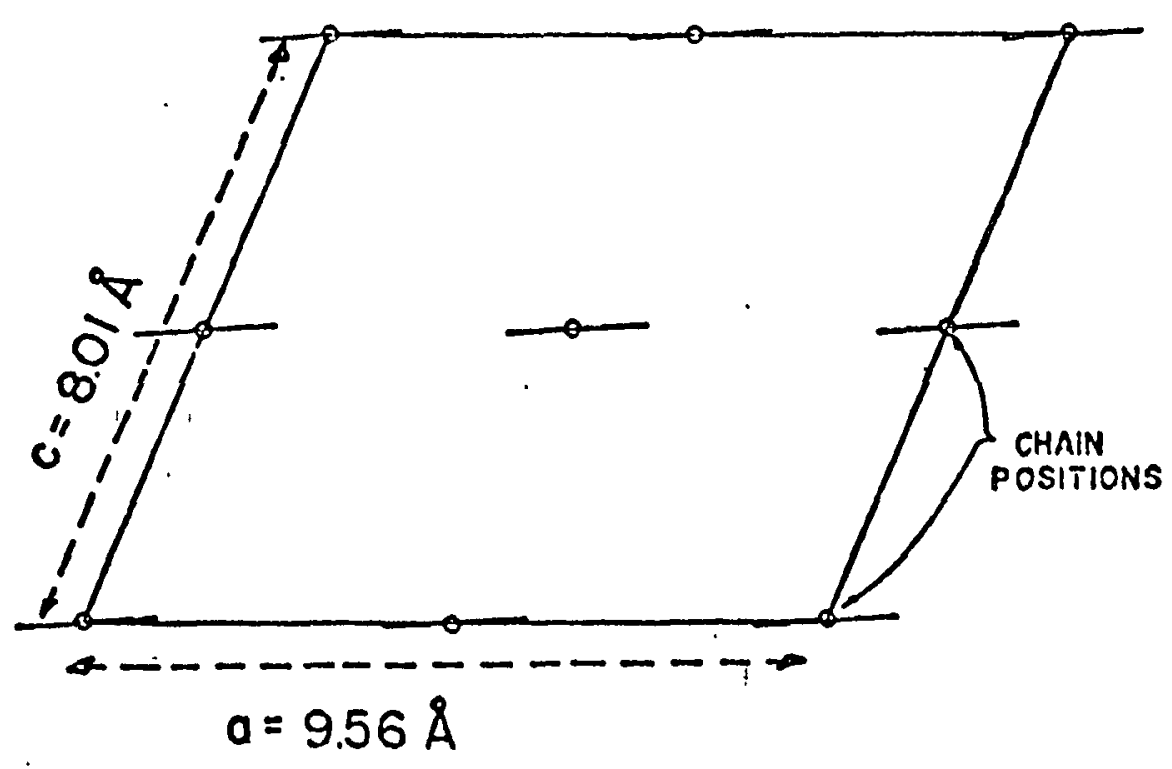

FIGURE 29. Unit cell dimensions of a single crystal of nylon 6 : 
regions is the same or less dense than that quoted for a 100 per cent amorphous polymer. $X$-ray evidence seems to indicate that no clear cut two phase system exists in highly drawn fibers but rather only degrees of order and disorder or a paracrystalline type structure (19) 23, 50). Even so, the existance of disordered, tie chain, amorphous, or flaw regions between the highly ordered regions seems evident to explain strength and fracture data. These amorphous regions most likely consist of: (a) chain segments that are incorporated in the two adjacent "crystalline-like" regions (tie molecules); (b) chain loops (polymer chains re-entering the same "crystalline-like" regions); and (c) chain ends. The tie molecule portion is only some fraction of the total. Meinel and Peterlin conducted studies with fuming nitric acid treatments of highly drawn polyethylene which they interpreted to indicate what fraction of polymer chains were tie chains $(13,51)$. In these studies, fumic nitric acid was assumed to attack polymer chains in the amorphous regions with the exception of the tie chains in these regions. Crystalline regions were not attacked. This resulted in two molecular weight species which were interpreted to correspond to a single crystalline lamellae and of two crystalline lamellae interconnected with tie chains. From the relative values of the molecular weight species, the fraction of tie molecules was estimated. These results indicated ratios of the number of crystalline polymer chains to amorphous tie chains between three and ten. Although the author does not agree completely with the interpretation of the results, this is much more realistic than the crystalline to amorphous density ratio value. In light of previous discussions of the fracture process occurring in the 
"weak link" or "critical flaw" regions, one can speculate that there most likely exists a distribution of disordered or amorphous regions throughout the fiber. However, all of these amorphous or tie molecule regions not being equivalent would most likely not be involved in the fracture process. In fact, only the weakest or most critical regions may contibute to the fracture process. The model for fracture derived by the author considers only those reqions which ideally contribute to the fracture process and calls them "critical flaw" regions, whereas all disordered regions are combined with the ordered regions and designated "crystalline-block" regions. From this discussion, one might expect even larger crystalline to amorphous tie chain number ratios than those reported by Peterlin (13) if we are considering the most critical tie chain regions and not merely the average region. Experimental evidence of larger ratio values was observed by Backman and DeVries in experiments on bulk semi-crystalline polymers $(33,45)$. In these experiments, EPR measurements of the number of free radicals formed in cutting and grinding of bulk semi-crystalline nylon 6 , nylon 66, polyethylene and polypropylene were combined with measurements of the surface area created during the process. Results of these studies indicated approximately $2 \times 10^{13}$ free radicals are formed per square centimeter of surface formed. (Values from these measurements varied between $6 \times 10^{12}$ to $4 \times 10^{13}$ spins $/ \mathrm{cm}^{2}$.) Considering two free radicals or spins per polymer chain, this results in $1 \times 10^{13}$ chains $/ \mathrm{cm}^{2}$. Explanation for this apparentiy small value of chains $/ \mathrm{cm}^{2}$ fractured during the degradation process was that cracks progress selectively through the 
critical amorphous flaw regions of the polymer around the more ordered crystalline regions. If we compare this value with the number of chains/ $\mathrm{cm}^{2}$ in a nylon 6 perfect crystal, we obtain a crystalline to amorphous tie chain number ratio of greater than 50 . Therefore, from the experimental evidence available, we might expect "cry-blk" to "critical flaw" tie chains number ratios to be somewhere between three and fifty in the model.

\section{The Chain Length Parameter "RL"}

The ratio of the original length of the "crystalline-block" region to the original length of the "critical flaw" region can at best be only speculated because of lack of concrete experimental evidence. Some evidence does exist, however, from smail angle $X$-ray studies as to the length of the ordered or crystalline regions. Small angle $X$-ray patterns result only where there occurs an inhomogeneity in the microstructure of the polymer. Interpretation of long period values obtained from such measurements in highly oriented fibers has varied. Early interpretations of this data was used as evidence for the length between crystalline and amorphous regions in the fringed micelle model $(52,53)$. Later analysis of the data by Dismore and Statton (19) indicated that long period data was evidence for chain folded regions. Chain folding, by definition, provides for a discontinuity at the folded surface. Regions included by folds are assumed to be crystalline and therefore have close to the maximim electron density. Thus, assuming a less dense adjacent region to the folded region, there will occur a major difference of electron density between these regions and the adjacent regions at the ends of the folds causing observation 
of the long period from small angle X-ray data. For nylon 6 and nylon 66 fibers, the long period has been reported to be approximately $100 \mathrm{~A}$ in length $(19,23)$. Length of the tie chains or amorphous region has been speculated mostly from crystallinity percentage although Peterlin (13) has interpreted results from fumic nitric acid treatments in polyethylene to give a crystalline region of $120 \mathrm{~A}$ and an amorphous tie chain region of $50 \mathrm{~A}$. If we assume the "crystalline block or ordered" regions are 100 per cent crystalline and the "flaw" regions 100 per cent amorphous, then the total sample crystallinity could be approximated by the original length ratios of the regions. Figure 30 shows this relationship. As these assumptions are probably not valid, i.e. the "cry-blk" regions are most likely not 100 per cent crystalline and the "critical flaw" regions are probably the least dense of all amorphous regions, estimates of crystallinity from such a relationship are probably high. Furthermore, the meaning of crystallinity is not clear. Crystallinity as judged from X-ray data (50) gives much different values than crystallinity from density measurements. For instance, the crystallinity as judged from the crystalline perfection index (CPI) for highly oriented fibers ranges from 50 per cent to 90 per cent whereas crystallinity from density measurements for the same samples ranges from 30 per cent to 50 per cent (19). Crystallinity measurements of nylon 6 in our 1aboratory give a CPI of approximately 75 per cent and a density crystallinity of 35 per cent (sample density $=1.42 \mathrm{gm} / \mathrm{cm}^{3}$ ) (23). Results from EPR bond rupture data also add to the speculation. Most researchers report the number of free radicals observed at fracture in nylon 6 as approximately $5 \times 10^{17}$ spins $/ \mathrm{cm}^{3}$ or $2.5 \times 10^{17} \mathrm{chains} / \mathrm{cm}^{3}$ 


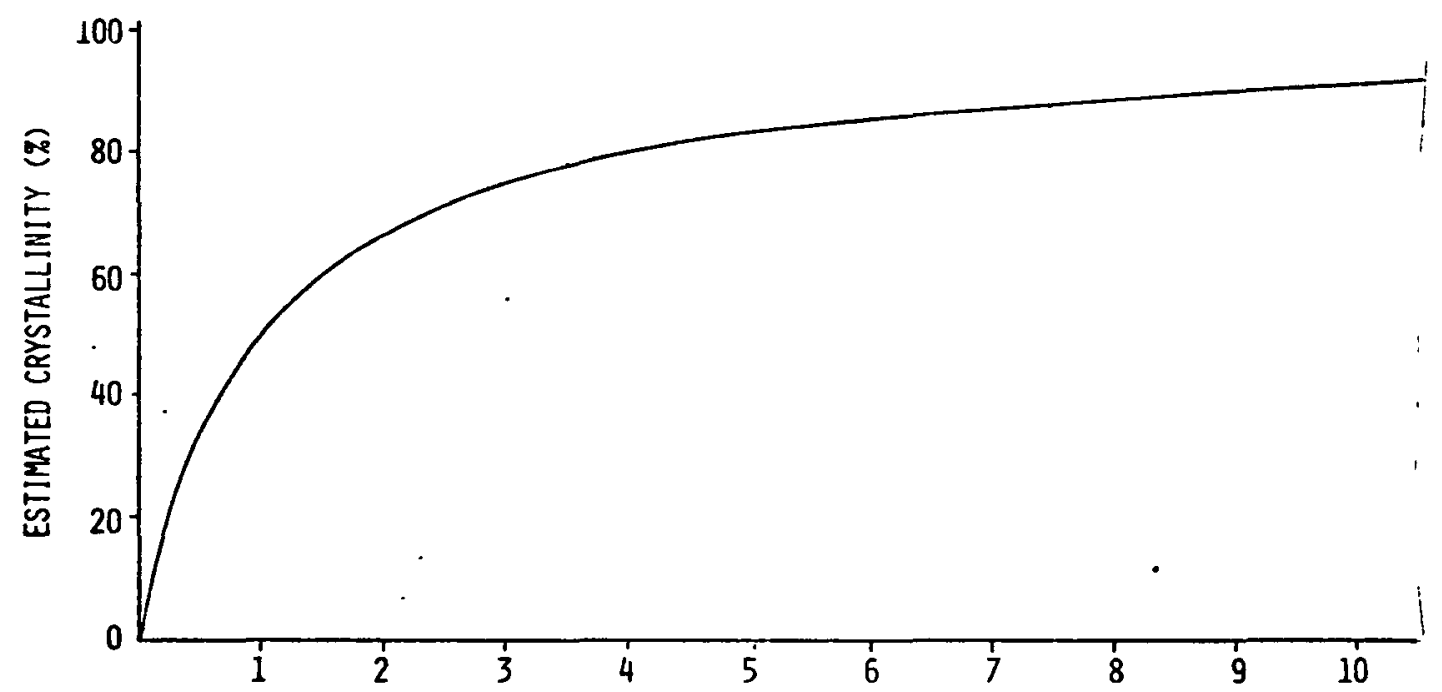

RL RATIO $=\frac{\text { ORIG LENGTH ORDERED REGION }}{\text { ORIG LENGTH FLAW REGION }}$

FIGURE 30. Relationship between crystallinity and original chain length, RL, if ordered regions were $100 \%$ crystalline 
$(9,29,42,54)$. If we assume EPR results give total possible tie chain ruptures and fracture occurs in every amorphous tie chain region and that these regions occur every 100A, then we would conclude that there was approximately $2.5 \times 10^{11}$ chains $/ \mathrm{cm}^{2}$ in each amorphous region.

This figure is almost two orders of magnitude lower than the values observed by Backman (45), and therefore the assumptions become suspect. The author will present results which indicate all three premises are possibly in error. From the above discussion the ratio of the original length of the "critical flaw" region is somewhat uncertain. Ratio values could be as small as two and as large as 100 depending on the number and structure of the fracture regions.

4. The Modulus of Elasticity of a Single Nylon 6 Polymer Chain "E

Treloar (55) has calculated the modulus of elasticity of a single nylon 66 chain based on a single chain area of $17.6 A^{2}$ as $2.84 \times 10^{7} 1 \mathrm{~b} /$ $\mathrm{in}^{2}\left(2 \times 10^{4} \mathrm{~kg} / \mathrm{mm}^{2}\right)$. The chemical composition of nylon 6 is the same as nylon 66 with a slightly different arrangement of the $\mathrm{CH}_{2}$ groups and the single crystal area is $17.7 A^{2}$. These similarities make use of the nylon 66 modulus value for nylon 6 as reasonable.

5. The Collision Parameter " $w_{0}$ "

This is one of the constants in rate process failure criterion and theoretically should have a value that approaches the thermal vibration frequency of the atom. Zhurkov (29) reports this value as $10^{12}$ to $10^{13}$ vibrations per second.

6. The Activation Energy "U ${ }_{0}$ "

The parameter $U_{0}$ has the dimensions of energy and in the model responds to the height of the activation barrier which must be overcome for breakage of the bonds responsible for strength in a nylon 6 
chain. The activation energy for bond dissociation is not the same as the bond dissociation energy and in general we would not expect to find a very close relationship between the two energies. However, in reaction of this type, i.e. separation or fracture of chemical bonds, the activation energy will be equal to or more positive than the heat of reaction (56). In the theory considered, the heat of reaction would correspond to the bond-dissociation energy required to rupture a nylon 6 chain at its weakest point. The bond dissociation energy for a carbon-carbon bond in a paraffin hydrocarbon chain has been determined by Geró to be $82.59 \mathrm{Kcal} / \mathrm{mole}(57)$. The weakest bond in the nylon 6 chain been theoreticaliy calculated to have a bond dissociation energy of approximately $15 \mathrm{Kcal} / \mathrm{mole}$ less than an ordinary $\mathrm{C}-\mathrm{C}$ bond (58). These values would place the bond dissociation energy for the weakest link of a nylon 6 chain at around $68 \mathrm{Kcal} / \mathrm{mole}$. The actual bond dissociation energy of this weakest link in a nylon 6 polymer may be lower than this value due to the additional "delocalization" of the binding electrons caused by the surrounding supermolecular structure. Significantly, lower values of the activation energy have been obtained from studying the effect of temperature on the rate of bond rupture on a macroscopic basis either by thermal or mechanical degradation. Zhurkov $(29,59)$ obtained activation energy values for mechanical degradation from polymer lifetime data, where the time-to-failure $\left(t_{b}\right)$ was assumed to be a kinetic rate process described by:

$$
t_{b}=\frac{1}{w_{0}} \exp \frac{U_{0}-y \sigma}{k T}
$$

where $w_{0}, U_{0}, \gamma, k$ and $T$ are as previously defined and $\sigma$ is the 
applied macroscopic stress. The energy of activation was then obtained from a plot of the in of time-to-failure versus one over the temperature for a constant applied macroscopic stress. From this data, he reported a value of $U_{0}$ as $45 \mathrm{Kcal} / \mathrm{mole}$ for nylon 6 . This value is very close to the value of $U_{0}$ of $43 \mathrm{Kcal} / \mathrm{mole}$ reported for nylon 6 (59) obtained from chemical bond rupture data in thermal degradation. From these values, Zhurkov concluded that there existed a direct connection between the kinetics of fracture of solids under action of mechanical stress and the rupture of interatomic bonds. This fact then led to the use of the Zhurkov equation, Equation 3 , for predicting chain scission. Roylance (30) in an effort to duplicate these values, conducted similar tests and concluded that the Zhurkov equation for chain scission (Equation 3) did not follow an Arrhenius relation. As the instanteneous chain scission did not seem to follow this rate process type treatment when macroscopic stress values were used, Roylance attempted to use a steady state chain scission relationship and obtained an activation energy value of $7 \mathrm{Kcal} / \mathrm{mole}$ for this process. However, at this point, he was convinced that the error in the Zhurkov work was due to use of a macroscopic stress value which was not proportional independent of time to the individual stresses on a polymer clain in the fiber. A most recent publication by the Russian author, Chevychelov (37), reports some work incorporating a distribution of stresses with rate process theory and indicates an activation energy of $57 \mathrm{Kcal} / \mathrm{mole}$ for nylon 6 necessary to fit experimental data. From the above discussion, one can expect that activation energy values obtained from studying the effects of temperature on the rate of bond rupture on a 
macroscopic basis either by thermal or mechanical degradation may not agree with the $U_{0}$ values for atomic or microscopic reactions due to the inhomogeneity of the stresses on the bonds.

7. The Activation Volume " $\gamma$ "

This constant is often referred to as the "activation area" even though it has the dimensions of volume. Theoretically one might think of this value as the area of a chemical bond times the distance necessary to separate the adjoining atoms to cause rupture. Perepelkin (47) states that while the value of the collision parameter $w_{0}$ is practically independant of the fiber structure, the activation volume $\gamma$ as determined from macromeasurements is a coefficient which must take into account the structure of the material and the nonuniformity of stress distribution in molecular chains. From this basis, he calculates $\gamma$ values for various structure considerations, stating that the closer to an ideal structure the smaller $\gamma$ becomes approaching a value on the order of a molecular volume. Table 2 summarizes these results for nylon 6 fibers.

\section{TABLE 2}

Activation Volume " $\gamma$ " calculated by Perepelkin (47) for nylon 6 Fibers $\left(\mathrm{cm}^{3}\right)$

\begin{tabular}{|l|l|l|}
\hline $\begin{array}{l}\text { Calculated for real } \\
\text { Real Fibers }\end{array}$ & $\begin{array}{l}\text { Calculated for Ideal1y } \\
\text { Ordered Fibers }\end{array}$ & $\begin{array}{l}\text { Calculated for Ideal1y } \\
\text { Ordered Fibers with } \\
\text { Molecular Interaction }\end{array}$ \\
\hline $107-219 \times 10^{-24}$ & $17-28.9 \times 10^{-24}$ & $1.02-17.1 \times 10^{-24}$ \\
\hline
\end{tabular}


Experimental values of " $\gamma$ " for nylon 6 were determined by Zhurkov $(29,47,59)$ from Equation 31 where the stress was varied at constant temperature and the results plotted in terms of in of the time-to-failure versus applied macroscopic stress. From this linear plot, the slope " $\gamma$ " was evaluated and reported by Perepelkin (47) as $206 \times 10^{-24} \mathrm{~cm}^{3}$. Zhurkov reported that an equivalent " $\gamma$ " value was obtained from a similar linear plot of rate of chain scission data for quasi-load rate tests using Equation 3 (29). Roylance (30) observed that the linear relationship between the in of the instantaneous chain scission and the applied macroscopic stress at constant temperature did not occur for other loading conditions, such as constant stress (creep) tests and even such a relationship was questionable for constant load rate tests. Further investigation by the author indicated that there might be such a linear relationship between the $\mathrm{In}$ of the steady state formation of free radicals $\left(\dot{C}_{S S}\right)$ and the applied macroscopic stress. (This approach was suggested by Zhurkov upon questioning his original observations.) This approach did seem to give linear relationships for nylon 6 subject to several types of loadings (fatigue, creep, and constant stress rate) as shown in Figure 31. However, a different slope and therefore a different " $\gamma$ " value seemed to be necessary for each type of tests. Comparison of such values are shown in Table 3. Comparison of the activation values obtained from these macromeasurements indicate a wide variation and are generally much larger than those theoretical values reported by Perepelkin in Table 2 for ideal fibers. Calculation of the distance necessary to rupture adjoining atoms in nylon 6 chain, assuming a bond area of $17.7 \times 10^{-16} \mathrm{~cm}^{2}(48)$ and the " $\gamma$ " values lijsted in Table 3 , gives 


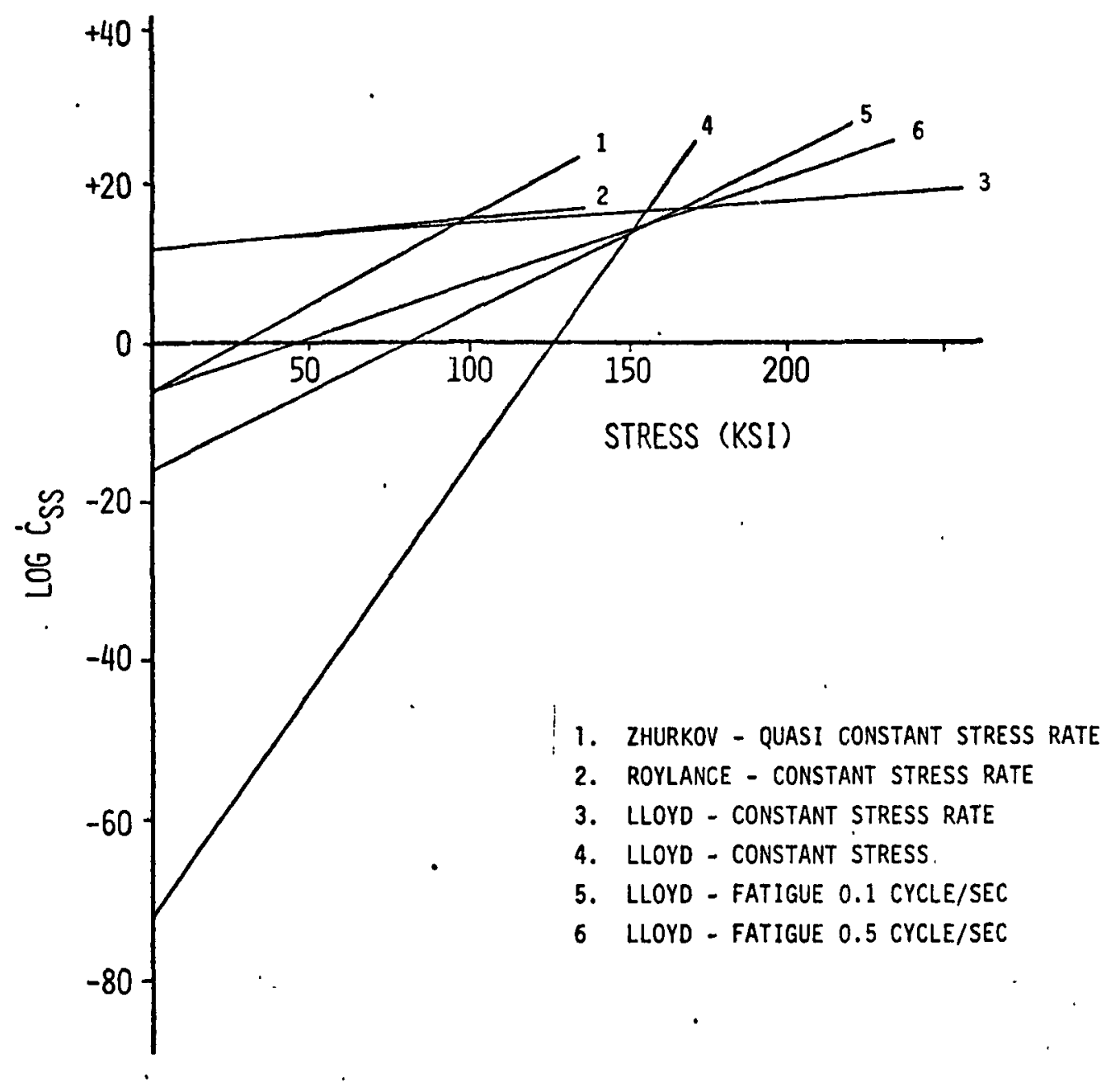

FIGURE 31. Linear relationships observed between the steady state chain scission rate, $C_{\text {sS }}$, and the applied macroscopic stress for Sarious loadings 
Table 3

" $\gamma$ " Values for Nylon 6 obtained from Figure 32

Type of Test

$\gamma\left(\mathrm{cm}^{3}\right)$

Source

Constant Load Rate

$300 \times 10^{-24}$

Zhurkov $(29)$ *

Constant Load Rate

$37 \times 10^{-24}$

Roylance (30)

Constant Load Rate

$33 \times 10^{-24}$

Lloyd

Constant Load

$800 \times 10^{-24}$

Lloyd

Fatigue 0.5 cycle/se

$180 \times 10^{-24}$

Lloyd

Fatigue 0.1 cycle/sec

$270 \times 10^{-24}$

Lloyd

$\star$ This value obtained from a replot of the Zhurkov data reported
in reference 29 and differs slightly from the value of $206 \times 10^{-24} \mathrm{~cm}^{3}$
reported by Perepelkin (47). 
values ranging from 2 to $45 A^{0}$. Peterlin reports a similar value of 0

$8 \mathrm{~A}$ from macromeasurement data (13) and states that this cannot possibly be considered an actual displacement. He further indicates such a value should be more on the order of $0.3 \hat{A}$. Assuming a bond area of $17.7 \times 10^{-16} \mathrm{~cm} .^{2}$ and a displacement of $.3 \times 10^{-8} \mathrm{~cm}$. necessary to cause fracture, one obtains an activation volume value " $\gamma$ " of $5.2 \times 10^{-24} \mathrm{~cm}^{3}$. In essence, the activation volume term as determined from macromeasurement data appears to have little if any meaningful value and does not even appear to be a constant.

From the discussion of the model parameters, one senses that the values that could be selected encompass a broad spectrum. As a result. of this observation, the basic approach for determination of the parameters was as follows: Those parameters that were best known, either from experiment or theory would be selected as base values. This included the distribution standard deviation value (S) obtained from EPR data, the modulus of elasticity $\left(E_{b}\right)$ for a single nylon 6 chain, and the rate theory collision parameter $\left(w_{0}\right)$. The other four parameters, $R C, R L, U_{0}$ and $\gamma$ were to be selected by comparison of the theoretical solution, using various combinations of the parameters, with experimentally observed data from constant strain rate and constant stress (creep) tests of nylon 6 \#1 at room temperature. Four curves, (two strain rate and two creep) were selected as the comparison standards. There was, of course, some expermental variation in tests. This is usually found to be particularly true of creep tests at loads where failure occurs in fairly short times. As a consequence, several tests of each type were run and the curve fit accomplished on an average of these tests. With these guidelines, parameter values at room 
temperature were then selected and the theoretical results plotted and compared with the experimental standards. To obtain the "best fit" of the experimental standards, over 150 different combinations of the parameters, $\mathrm{RL}, \mathrm{RC}, \mathrm{U}_{0}$, and $\gamma$ were computed and plotted as well as some variations of the parameters $S, E_{b}$ and $w_{0}$. The ratio of number of chains, RC, was varied from 10 to 50 , the ratio of original lengths $\mathrm{RL}$, was varied from 1 to 10 , the activation energy, $U_{0}$, was varied from $45 \mathrm{Kcal} / \mathrm{mode}$ to $82 \mathrm{Kcal} / \mathrm{mole}$ and the activation volume $\gamma$ was varied from $0.5 \times 10^{-24}$ to $200 \times 10^{-24} \mathrm{~cm}^{3}$. In order to more easily compare experimental free radicals versus time curves with theoretical free radical or chain scission versus time data, a value of $1 \times 10^{13}$ chains/ $\mathrm{cm}^{2}\left(2 \times 10^{13}\right.$ spins $\left./ \mathrm{cm}^{2}\right)$ was selected as the total number of chains $/ \mathrm{cm}^{2}$ in the "critical flaw" region. After considerable time and effort, a group of parameters was selected as the "best fit" with the knowledge that moderate variations would not substantially degrade and possibly would improve the comparison with the experimental standards of nylon 6 \#1 at room temperature. (The creep test standard curves were found to be the most critical to fit). Figures 3 ? and 33 show experimental stress, strain and bond rupture data (solid lines) and theoretical predictions of the same data (dotted lines) for constant stress rate and constant stress (creep) tests for nylon $6 \# 1$. The experimental standard curves are marked "STD". Once these parameters were determined, they were used to predict behavior at other strain rates and creep at other stress levels for nylon 6 \#1 at room temperature. The same parameters were used to predict stress, strain, and bond rupture behavior for different loadings, i.e. constant stress rate, cyclic stress fatigue, (at several frequencies), constant strain (stress 

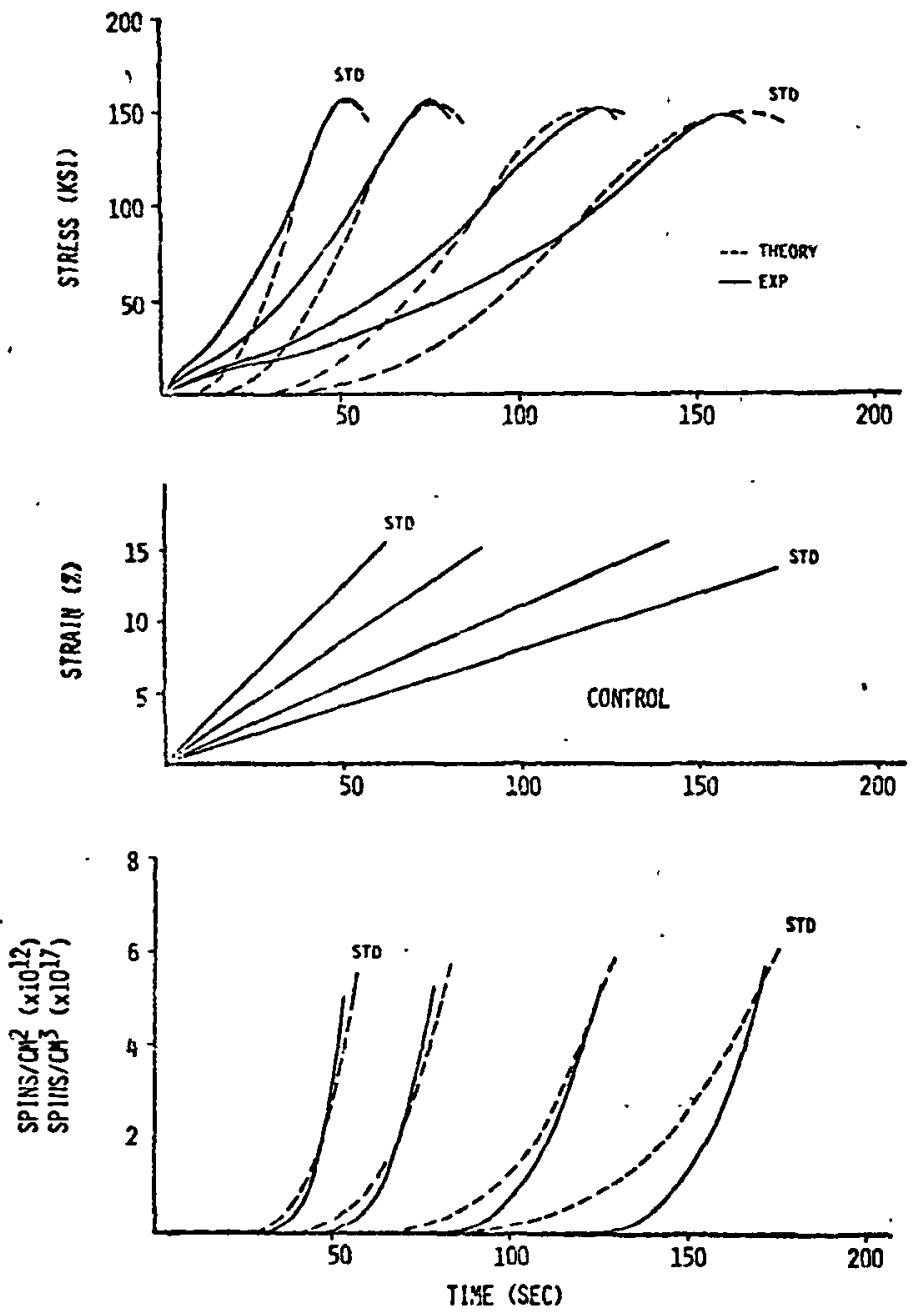

FIGURE 32. Constant strain rate tests for nylon-6 \#1 at room temperature. (Theory Spins $/ \mathrm{cm}^{2}$ ).
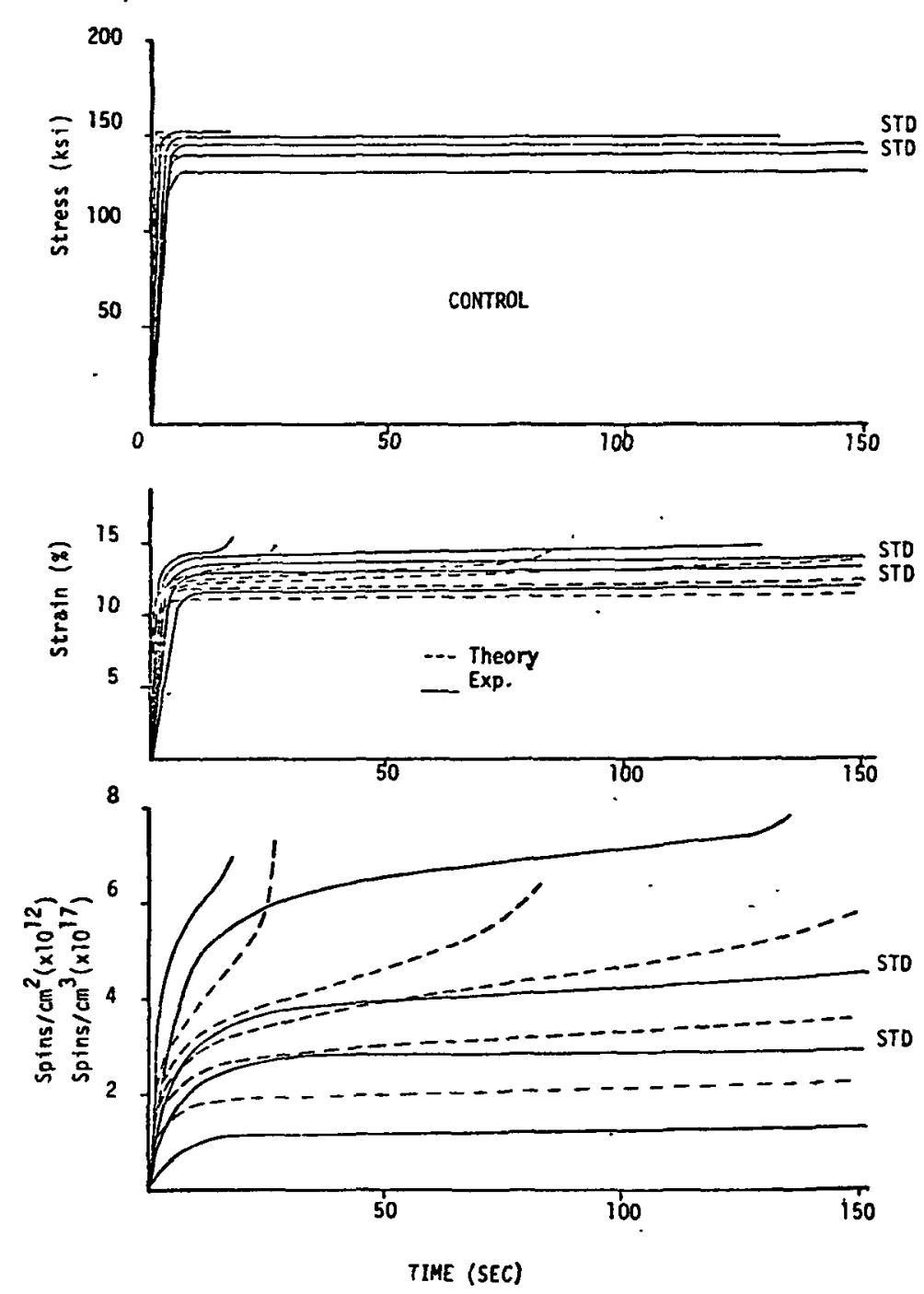

FIGURE 33. Constant stress (creep) tests for nylon 6 \#1 at room temperature. (Theory - Spins $/ \mathrm{cm}^{2}$ ). 
relaxation) and step strain tests for the same nylon and temperature. The predicted behavior and experimentally determined behavior for comparison are shown in Figures 32 through 39. The agreement between the model predictions and experiments was truly gratifying. Considering the complexity of the polymer fiber microstructure, the relatively unknown value of the parameters and the task of relating atomic occurences to. macroscopic observations, the author felt that even a quantitative relationship between theory and experiment would have been considered a success. It will be noted from the figures that the agreement between predicted and experimental behavior is much better than qualtitative. It was, in fact, generally found that in most aspects the comparison was nearly as good as between any two experimental curves of identical input loads. The exciting aspect of thesa correlations was that parameters establish from simple strain rate and creep tests were successful in predicting behavior even in low cycle fatigue tests. The "best fit" parameters used in the comparisons for nylon 6 \#1 at room temperautre were:

$$
\begin{aligned}
& S=1.25(10 \text { per cent of the distribution mean strain) } \\
& R C=30 \\
& R L=5 \\
& E_{b}=3 \times 10^{7} \mathrm{lb} / \mathrm{in}^{2} \\
& W_{0}=10^{13} \mathrm{vib} / \mathrm{sec} \\
& U_{0}=67.5 \mathrm{Kcal} / \mathrm{mole} \\
& \gamma=5 \times 10^{-24} \mathrm{~cm} . \\
& T=295^{0} \mathrm{~K} \text { (room temperature } 22^{\circ} \mathrm{C} \text { ) } \\
& W=1 \times 10^{13} \text { chains } / \mathrm{cm}^{2}
\end{aligned}
$$



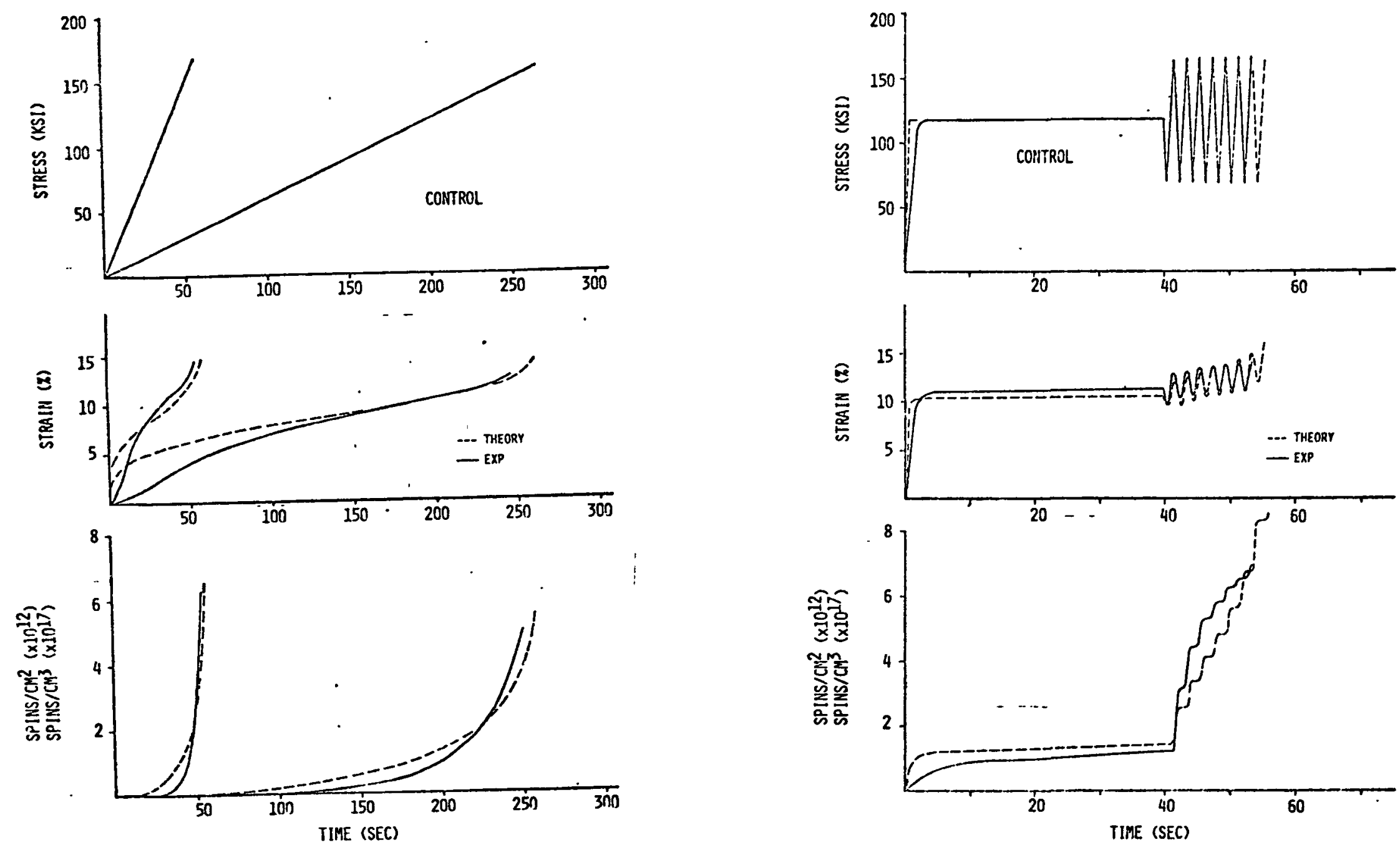

FIGURE 34. Constant stress rate tests for nylon 6 \#1 at room temperature. (Theory - Spins/ $\mathrm{cm}^{2}$ ).

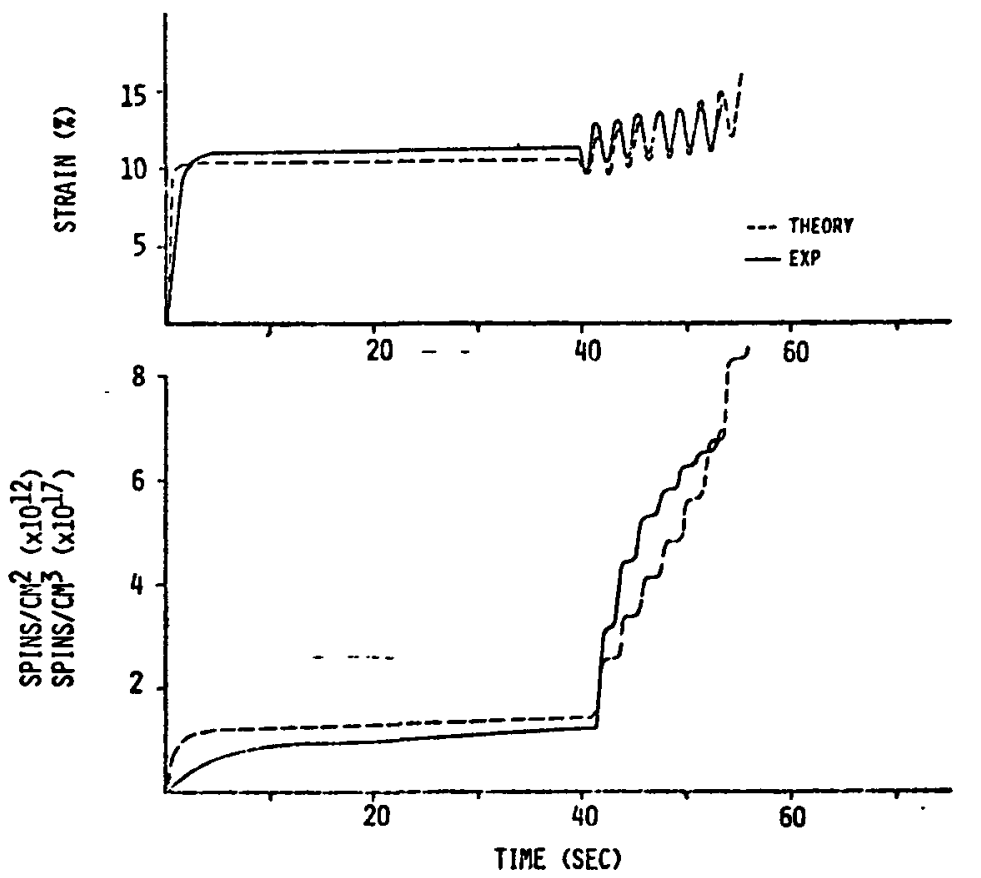

FIGURE 35. Cyclic stress fatigue $(0.5 \mathrm{cycle} / \mathrm{sec})$ test for nylon 6 \# at room temperature. (Theory - Spins $/ \mathrm{cm}^{2}$ ). 

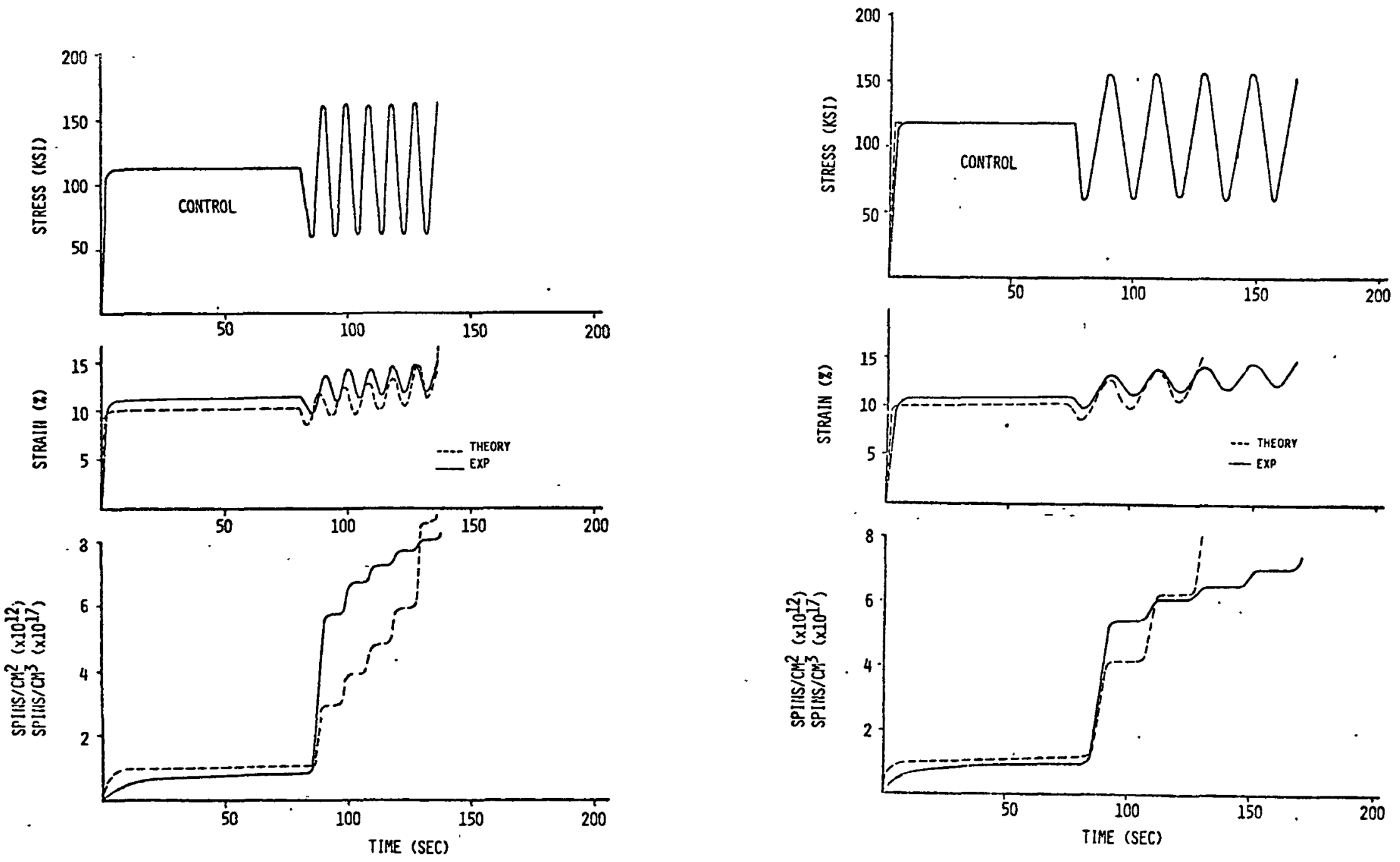

FIGURE 36. Cyclic stress fatigue $(0.1 \mathrm{cycle} / \mathrm{sec})$ test for nylon $6 \# 1$ at room temperature. (Theory - Spins $/ \mathrm{cm}^{2}$ ).

FIGURE 37. Cyclic stress fatigue (0.05 cycle/sec) test for nylon 6 \#1 at room temperature. (Theory - Spins $/ \mathrm{cm}^{2}$ ). 

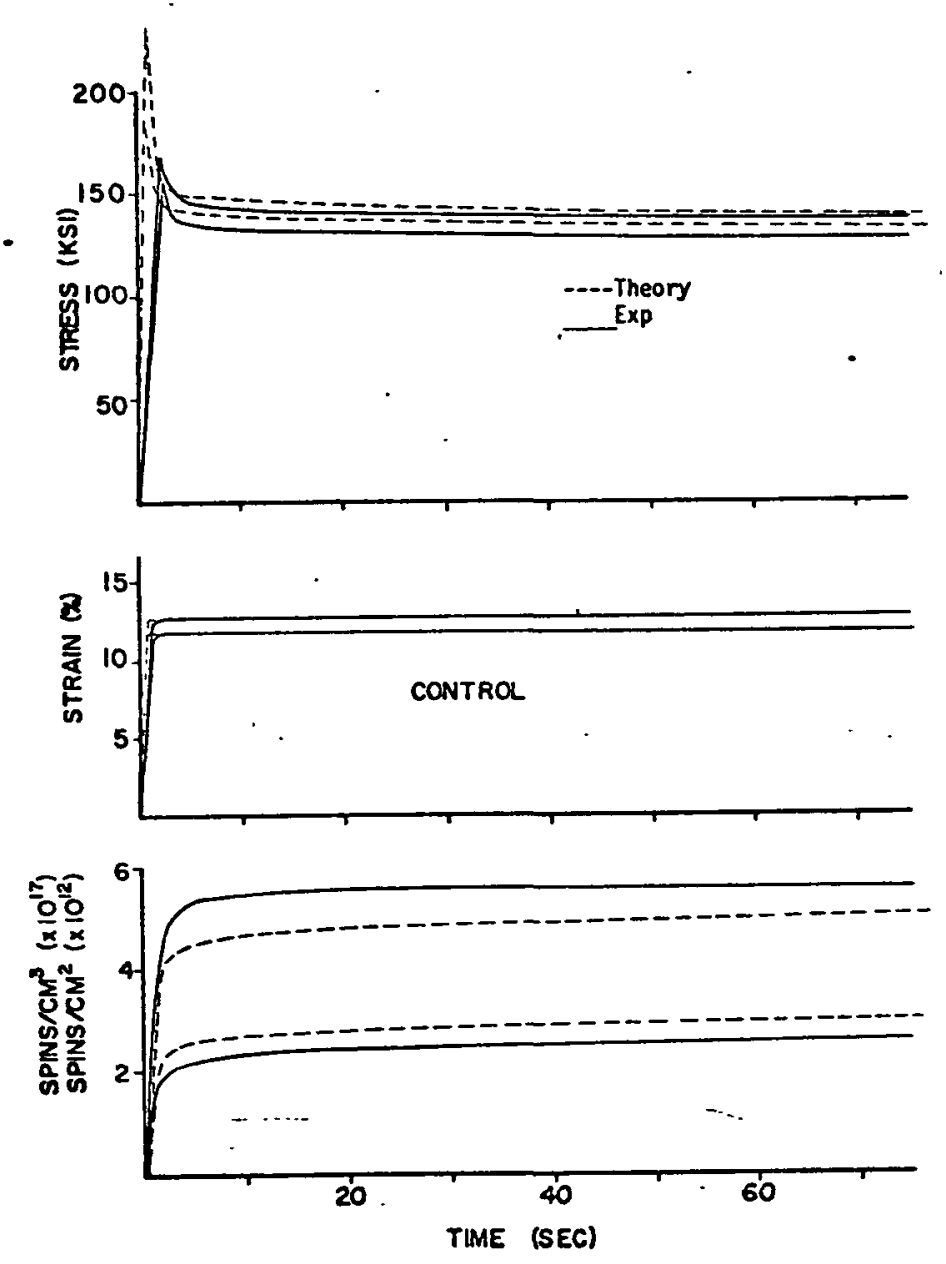

FIGURE 38. Constant strain (stress relaxation) tests for nylon 6\#1 at room temperature. (Theory - Spins $/ \mathrm{cm}^{2}$ ).
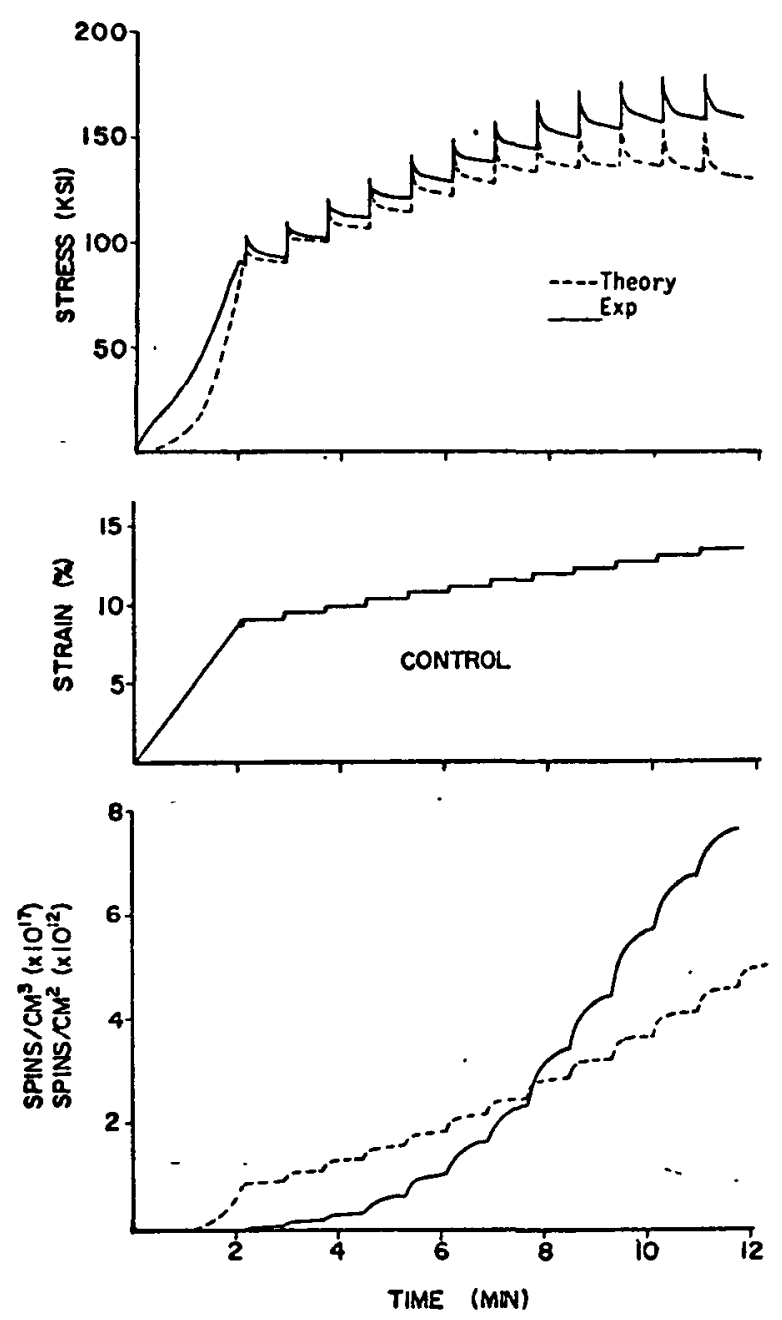

FIGURE 39. Step-Strain test for nylon 6 \#1 at room temperature. (Theory Spins $/ \mathrm{cm}^{2}$ ). 
5.4 DISCUSSION

Some of the differences and observations between the theoretical predictions and experimental tests are worthy of comment.

1. The early portion of the stress curves in the constant strain rate tests (Figure 32 ) and analogously the strain for the constant stress rate tests (Figure 34) do not match exactly. In this portion of the loading curve many of the tie chains are not taut and one might expect some siqnificant entropic forces due to chains unkinking, uncoiling or generally straightening themselves to a taut position. Pechhold (60), assuming several modes of viscoelastic relaxation and calculating entropic forces has indeed had some success in explaining the stress-strain behavior in this region. As the model by the author does not include such forces the resulting theoretical predictions in fact do support the ideas of Pechhold in that early portions of the experimental stress-strain curve (Figures 32 and 34) cannot be duplicated by a model that accounts for chain scission only.

2. It will also be noted that for the creep and fatigue experiments (Figures 33, 35-37), the initial growth of broken bonds is greater under a large experimental step load than is predicted theoretically. This is not completely understood but may possibly be treated by slight adjustments in the kinetic. parameters, particularly if account is taken of the fact that some secondary slippage may occur at the higher stress levels. Figure 40 shows the results of adding stress relaxation to the same parameters used to predict the creep tests of Fiqure 33. 

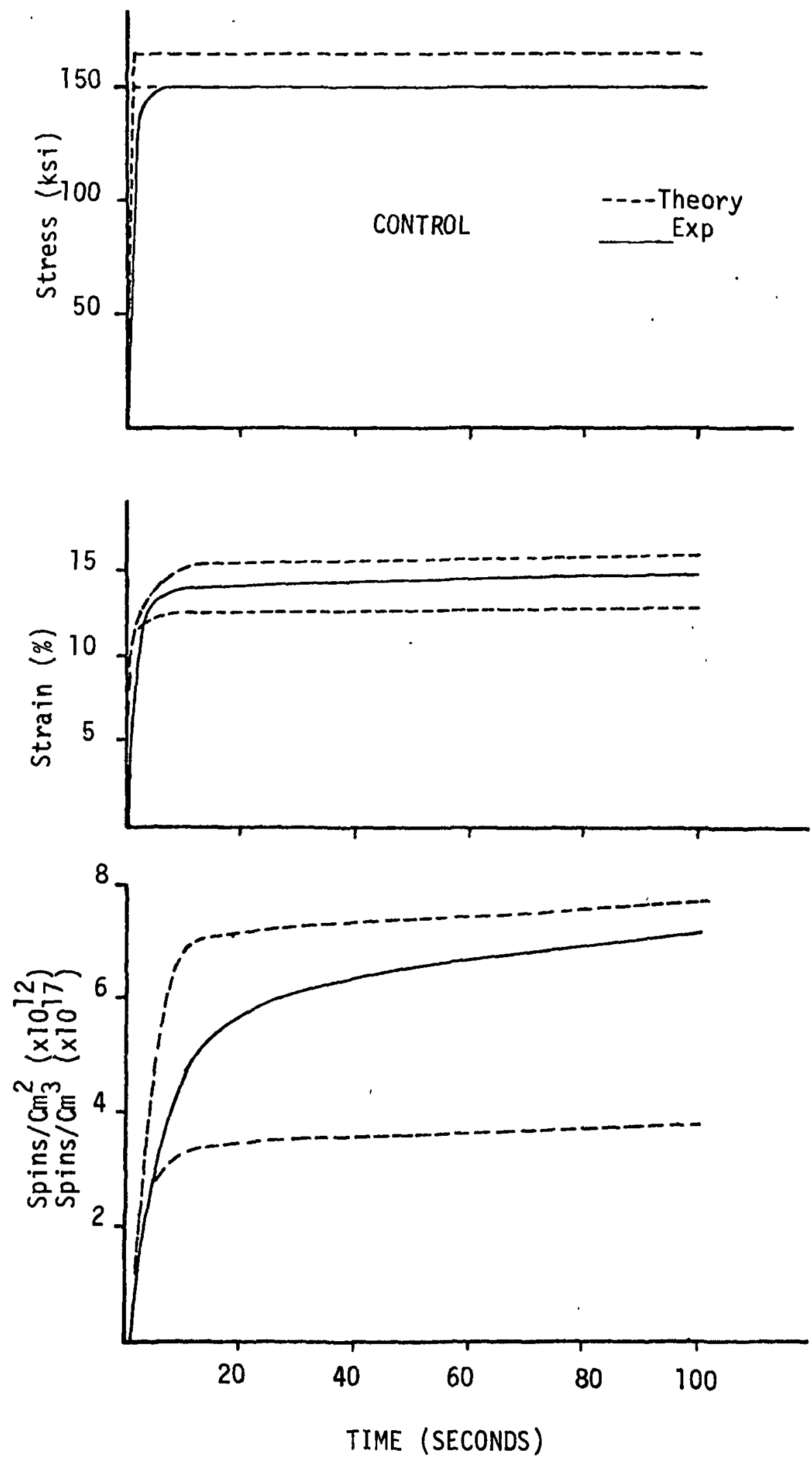

FIGURE 40. Creep test with stress relaxation added to theoretical parameters. (Theory--spins $/ \mathrm{cm}^{2}$ ) 
Note that such an addition, if included with slight parameter adjustments, may indeed improve the comparison between theoretical and experimental chain scission results. (One chain scission equals two spins.)

A probable explanation is that the sudden application of a large step load may cause initial chain scission in the flaw regions other than the "critical flaw" regions. After the initial large step, the stress in these regions would be relaxed by the more rapid degradation of the adjoining "critical flaw" regions and only the critical regions would contribute significantly to chain scission from that point in time. Some evidence of this possibllity can be observed from the fatigue tests, (Figures 35-37) where the chain scission results correlate very well except for the first fatigue step.

A third explanation might be conjectured from a changing "effective distribution" with the rate of load application. A rapid step load would allow little opportunity for unfolding or unkinking of chains and therefore might cause premature chain rupture in certain tie molecules. This explanation is closely linked with the first explanation of stress relaxation effects. Such an effect could possibly explain the greater total chain scission observed in most of the fatigue tests although this might also be explained by the initial contribution of other than "critical flaw" regions to the total.

Another major difference between theory and experiment is the application of the step loads. Theoretically, a true step 
load could be applied; experimentally the load application was never instantaneous but rather limited by the dynamic response of the equipment. This is evidenced by the comparison of the stress curves in the constant strain (stress relaxation) results of Figure 38 . The initial theoretical stress is much greater than the experimental stress. This is most likely due, in part, to the theoretical instantaneous step load which results in the higher instantaneous stress values. The effect of chain slippage, i.e. partial pull out or unkinking, prior to or in conjunction with chain scission under high step strains (or loads), could also explain the lower experimental stress levels. This and other differences between experimental and theoretical chain scission results and stress levels observed in stress relaxation tests (Figure 38) and the step strain test (Figure 39) can be explained in a similar manner to differences observed in creep and fatigue tests. Even with such differences, the overall correlation of experiment and theory is most satisfactory and the effect of corrections for short time loadings is regarded as small based on the good agreement obtained from the model by neglecting them.

3. The role of chain scission with regard to stress relaxation has been a subject of much debate. Certainiy stress relaxation can be attributed in a large portion to viscoelastic time effects, i.e. chain slippage (secondary bond failure), unkinking, uncoiling or unfolding; but what portion, if any, and at what level does chain scission contribute to such effects? Results, 
from experimental and theoretical constant strain tests, for strain levels above six per cent, (Figure 38 ) show that for these highly oriented fibers, stress relaxation can almost wholly be accounted for by chain scission. However, viscoelastic stress relaxation effects are observed in experimental tests at strain levels below six per cent where negligible chain scission and no stress relaxation is predicted by the theory. This correspondance can be clearly observed in the step strain test of Figure 39.

4. Macroscopic failure in these fibers might logically be divided into three stages of development:
a. Fracture initiation
b. Slow crack growth
c. Rapid crack propagation

The stages are characterized by different processes. Fracture initiation describes the period from the beginning of the mechanical excitation of the sample to the formation of a crack which is large enough to influence its own growth rate. In these high-strength fibers apparently certain potential flaw sites are created during manufacturing, drawing, special treatments, etc. which can act as fracture initiation sites. These sites are distributed thoughout the fiber and are composed, in part, of tie molecules linking the "crystallinelike" regions together. The relative number and arrangement of these tie chains determines which sites might be considered "critical flaw" sites. In the first stage of fracture initiation many flaw sites may be involved, however, due to 
the more rapid growth rate of the "critical flaw" sites, the stresses in other sites decrease and the "critical flaw" sites contribute almost wholly to the chain scission process. This is then considered the second stage of the fracture process, i.e. the "slow crack" growth process. It is in the average of these sites that chain scission is predicted by the theoretical model. However, as the size and shape of the developing "cracks" increase in growth rate, certain of the flaws become "more critical" than the others and attain an unstable (Griffith) size, thus entering the third and final period of development of macroscopic failure. This third stage by its very nature, must be more localized and at some point in time one or at the most a few of these "most critical" flaws dominate the fracture process and rapid crack propagation takes place causing catastrophic failure of the sample. This exact catastrophic failure point is not predicted by the model as it assumes all "critical flaw" regions contribute to the fracture process until total destruction of all tie molecules is accomplished. This is, of course, too ideal but even so the point of catastrophic failure can be approximated from the model in its present form. In the comparison of theoretical and experimental results shown in Figures 32-39, the theoretical fracture point was chosen by comparison with the corresponding experimental fracture point. Even so, the difference between this point and the theoretical fracture point, i.e. the point of total theoretical tie molecule destruction, was less than 
five to ten seconds for stress controlled tests, Figures 33-37. For strain controlled tests, such as Figures 32 , 38 and 39 , the catastrophic fracture point occurred a few seconds after the maximum stress level was exceeded. As this level is predicted quite accurately by the theoretical curves, the macroscopic fracture point for strain controlled tests can be estimated quite readily. Therefore, macroscopic fracture appears to occur catastrophically at a point where the micro-cracks in one or a few of the "critical flaw" regions reach a critical value. The proposed model not only predicts microscopic failure or slow crack growth due to chain scission, but can be used to estimate with fairly good accuracy the catastrophic macroscopic fracture point.

5. The model predicts failure in terms of the number of tie chains per unit.area while the experimental values are in terms of the number of free radicals per unit volume of material in the EPR spectrometer cavity. In order to compare the two, a knowledge of the amount of microcrack or flaw area per unit volume is required. Such information is currently not completely available and is a subject of current investigation. Some interesting tentative speculation is, however, possible from the present study. First, it will be noted that all the tie chains in the model were not ruptured at the corresponding experimental catastrophic fracture point. Estimates of the total number of tie chains can be made from 
both experimental and theoretical observations of tests completed in this study. From the experimental histogram plots shown in Figures 21 through 24 and assuming a symmetrical Gaussian distribution, the magnitude of the missing portion or tailing side of the distribution can be made. From such analysis, it is estimated that between 50 and 90 per cent of the total number of tie molecules are observed at fracture by EPR techniques (depending on the temperature, distribution, effect of plasticizers, etc.) Peterlin (61), however, has indicated that the tailing side of the distribution may be somewhat artifact due to a selective failure process proceeding through only the "most critical" of the flaws after the point of maximum load capacity has been exceeded. This concept was discussed above and certainly appears reasonable. However, if such a process was taking place, we might expect the tailing side of the distribution plots to deviate somewhat from a normal or gaussian distribution. Indeed such a deviation has been noted in probability plots of the experimental histogram data especially at high temperatures where more of the tailing side is present. From such observations, we might then expect the number of tie molecules observed at fracture to be somewhat less than the 50 to 90 per cent estimated value.

Comparing theoretical chain scissions (spins) at the fracture point from Figures $32-37$ and 39 , with the total 
number of assumed tie molecules $\left(1 \times 10^{13}\right.$ chains $/ \mathrm{cm}^{2}$ or $2 \times 10^{13}$ spins $/ \mathrm{cm}^{2}$ ) we observe only 30 to 40 per cent of the total possible chain scissions occur at the catastrophic fracture point. From variation of parameter studies, the author has determined that this percentage can be altered fairly significantly by slight adjustments in the model parameters. Figures 41 and 42 show one such modification for nylon 6 \#1 at room temperature where between 50 and 70 per cent of the total tie molecules are estimated to undergo chain scission at the catastrophic failure point. Model parameters for this case were $S=1.25, R C=10$, $R L=3.8, E_{b}=3 \times 10^{7} 1 \mathrm{~b} / \mathrm{in}^{2}, w_{0}=10^{13} \mathrm{vib} / \mathrm{sec} ., U_{0}=69 \mathrm{Kcal} / \mathrm{mole}$, $\gamma=10 \times 10^{-24} \mathrm{~cm}^{3}, W=1 \times 10^{13}$ chains $/ \mathrm{cm}^{2}$. From such observations, it is the author's opinion that between 45 and 80 per cent of the total tie molecules in the critical flaw regions are observed by EPR techniques and this fraction depends on several factors, such as temperature, rate and type of loading and effective distribution width. From the above discussion, researchers should take care in predictions or observations based solely on chain scission fracture point data obtained by EPR techniques.

Second, the absolute number of tie molecules in the critical flaw regions and the number of such flaw sites in a unit volume is a question of much interest. Although the important structural parameters in the model are ratio values only, i.e. $\mathrm{RC}=$ the ratio of polymer chains in the "cry-blk", region to the number of tie chains in the flaw region and $R L$ 


$$
\begin{aligned}
& A U S=B-H= \\
& \text { iiLK }
\end{aligned}
$$


$=$ the ratio of the original length of the "cry-blk" region to the original length of the flaw reqion, some interesting observations can be made from the model concerning the above question. Speculating on the mechanism of fracture and correlating it with experimental observations, we might envision the microstructure of the fiber to consist of disordered or amorphous sites distributed in some manner throughout the fiber volume. Some of these sites undoubtedly may by "weak link" or "critical flaw" sites due to the number or arrangement of the polymer chains in the region. As fracture begins, chain scission in the "critical flaw" sites relieves the stresses in adjoining and/or the next most critical sites. Fracture continues among all the "critical flaw" regions up to the point where one or a few such flaws become more critical than the others and catastrophic failure occurs following a path though a very small percentage of the total active flaw regions. This leaves most of the active flaw areas intact but altered by partial tie chain rupture. If the fracture pieces are subsequently tested, it is expected that they will contain similar but slightly less critical regions. During the initial loading, tie chains have been ruptured in these regions as we1l. However, they have not passed through that portion of their distribution where the load-carrying capacity starts to drop off. As a consequence, increased load or an additional time increment at the same load is required to cause the second rupture. It should be noted, however, that number of bonds ruptured during the second 
loading might be expected to be significiantly less than during the critical loading. This is, of course, due to the fact that some of the taut tie chains were broken in the initial loading. As a consequence, one might expect the engineering modulus of toughness due to dissipation of energy during the remaining bond rupture to be less for these secondary segments. Both these latter observations are consistant with the experiment.

In the model, al1 "critical flaws" are assumed to be represented by an average region. In this case, one might expect at a given time and for a given loading all the regions to be equally degraded and rupture to occur simultaneously in all these regions throughout the sample. Quite obviously such a situation is too ideal and not consistent with experimental observation. While these flaw regions are very similar, otherwise how might one explain the uniform bond rupture throughout the sample, at least some difference in these regions is to be expected. Therefore, one, or in exceptional cases a very few, will reach the critical size at which catastrophic failure occurs leaving the remaining flaw areas intact. In this case, the stress-strain behavior of the sample could be approximated reasonable well by an average "critical flaw" region.

From the theory, a chain ratio value, $\mathrm{RC}$, of 30 and an original region length ratio, $\mathrm{RL}$, of five was selected as "best fit" parameters. Variations of the data to determine 
the range of acceptable parameter values without substantialiy degrading the comparison with experimental results indicate possible acceptable for $R C$ from 20 to 40 and $R L$ from four to ten. The arbitrary base number for total tie molecules in the "critical flaw" regions was $1 \times 10^{13}$ chains $/ \mathrm{cm}^{2}$. Depending on the ratio RC and the number of chains $/ \mathrm{cm}^{2}$ in the "cry-blk" regions, this absolute value could vary substantially. If we speculate that the total number of chains in the "cry-blk" region. lies somewhere between $2-5 \times 10^{14}$ chains $/ \mathrm{cm}^{2}$, then using the range of theoretical "RC" ratio values, we estimate the total tie molecules in the critical flaw region to be between $0.5-2.5 \times 10^{13}$ chains $/ \mathrm{cm}^{2}$. If EPR techniques, as discussed above, record approximately 60 per cent of the total spins in the flaw regions, this would give a range of $0.3-1.5 \times 10^{13}$ chains $/ \mathrm{cm}^{2}$ predicted theoretically at the fracture point. Comparing these values with experimental fracture values of $3-6 \times 10^{17}$ chains $/ \mathrm{cm}^{3}\left(6-8 \times 10^{17}\right.$ spins $/ \mathrm{cm}^{3}$ ) results in approximately $10^{4}$ to $10^{5}$ square centimeters of crack area in a cubic centimeter of sample material at fracture.

It is interesting to note that Zhurkov, Kuksenko, and Slutsker (62) have interpreted $X$-ray data in terms of size and density of microcracks. Their work was on high-strength oriented nylon 6 and other films but the strength of these films approaches that of the fibers studied here. They reported at fracture $10^{15}$ to $10^{16}$ microcracks per cubic centimeter with an average crack size $250 \mathrm{~A}$ in diameter. This would correspond to 
$10^{3}$ to $10^{4}$ square centimeters of crack area per cubic centimeter of the sample material which agrees quite well with the estimation from the authors results.

Another approach to correlating chains $/ \mathrm{cm}^{2}$ in a fracture path to experimentally observed chains $/ \mathrm{cm}^{3}$ from EPR results has been to assume the total length of crystalline and amorphous regions to be given by small angle $X$-ray data. Long period results from such data under this interpretation indicate a combined length of approximately 100A for highly oriented nylon fibers $(19,23)$. If we assume chain scission occurs in every region, as many author's have, we would estimate, using EPR fracture results of $3-4 \times 10^{17}$ chains $/ \mathrm{cm}^{3}$, that the number of chains $/ \mathrm{cm}^{2}$ fractured in the amorphous regions to be $3-4 \times 10^{11}$ chains $/ \mathrm{cm}^{2}$. If this is sixty per cent of the total chains in that region, then the total tie molecules would only be $5-7 \times 10^{11}$ chains $/ \mathrm{cm}^{2}$. Comparing this with the estimated total number of tie molecules from this study, i.e. $0.3-1.5 \times 10^{13}$ chains $/ \mathrm{cm}^{2}$, we can estimate that only $1 / 6$ to $1 / 30$ of the regions are active. This might be explained by various means: (a) it could be that the parameters used in the model are in error. For example, perhaps the assumed number of tie chains in the flaw regions are in error or their strength is much different than the value used. Although this might be the case, the values used do have some basis. (b) another explanation, which appears most likely, is the one indicated above, i.e. either the distance between tie regions is much more than $100 \AA$ or only those separated by several 
hundred angstroms are critical. In either case the close correspondence of the predicted and experimental values for stress-strain behavior would lead us to believe the bulk of the amorphous material indicated by crystallinity measurement lies in these critical regions with the other "amorphous regions" behaving elastically more or less as crystalline blocks. Evidence from the model parameter RL leaves much room for speculation on the meaning of the $100 \AA$ unit. Ratio values of RL were theoretically predicted to be between four and ten. If from the above analysis, active flaw regions occur every 600 and $3000 \AA$, then the length of the critical flaw region would lie somewhere between 60 and $700 \AA$. The 0 60A figure is readily compatible with current thinking, although 0 the $700 \mathrm{~A}$ value seems high in relation to current knowledge of the fiber microstructure. Crystallinity estimates from the RL ratios, al though very speculative, can be obtained from Figure 30 and indicate the sample to be between 80 to 90 per cent crystalline. The crystallinity was approximated from this ratio by assuming the crystalline blocks as totally crystalline. As this is most likely not the case, this crystallinity value should be high.

6. In the above four statements the author has attempted to critically analyze differences between the theoretical and experimental data, and evaluate the microstructure parameters used in the model. Although some problems may exist, these parameters are reasonable. The "best fit" values and range obtained for the kinetic rate paramters $U_{0}$ and $\gamma$ were also 
reasonable. The activation energy $U_{0}$ had a "best fit" value of $67.5 \mathrm{Kcal} / \mathrm{mole}$ and a range of $65-75 \mathrm{Kcal} / \mathrm{mole}$. These values agree readily with the theoretical value of a single nylon 6 polymer chain estimated in section 5.3 to be approximately $68 \mathrm{Kcal} / \mathrm{mole}$. Likewise the "best fit" activation volume, $\gamma$, value of $5 \times 10^{-24} \mathrm{~cm}^{3}$ and range of $3-15 \times 10^{-24} \mathrm{~cm}^{3}$ was very acceptable in terms of molecular considerations. For instance, if a single bond was assumed to extend $0.3 \AA\left(0.3 \times 10^{-8} \mathrm{~cm}^{3}\right)$ at fracture, and the $\gamma$ range of theoretical values are used, the effective bond area of a single chain would lie between 10 and $50 \mathrm{~A}^{2}$. For the "best fit" $\gamma$ value, assuming $0.3 \AA$ extension, the effective area of a single chain is $16.7 A^{2}$ which is very close to the $17.7 A^{2}$ theoretical value of a perfect crystal. A summary of "best fit" values, their theoretical determined range from the model and the potential range from previously known data is summarized in Table 4. The theoretical range values were obtained by selectively varying the parameters and comparing the variations with experimental data. The limiting values chosen were those range of values which the author considered from this analysis might be used to predict the expermental results without significant degradation of the comparision. The "best fit" parameters, however, were considered to be the most compatible with the experimental results. 
Table 4

Model Parameters

\begin{tabular}{|c|c|c|c|}
\hline Parameter & Best Fit" Value & Theoretical Model Range & Potential Range \\
\hline S (\%strain) & 1.25 & 1.25 & no available data \\
\hline $\mathrm{RC}$ & 30 & $20-40$ & $3-50$ \\
\hline RL & 5 & $4-10$ & $2-100$ \\
\hline$E_{b}\left(1 b / i n^{2}\right)$ & $3 \times 10^{7}$ & $3 \times 10^{7}$ & $2.84 \times 10^{7}$ \\
\hline$w_{0}\left(\sec ^{-1}\right)$ & $10^{13}$ & $10^{13}$ & $10^{12}-10^{13}$ \\
\hline$\left.U_{0} \frac{(\mathrm{Kcal}}{\text { mole }}\right)$ & 67.5 & $65-75$ & $43-82$ \\
\hline$\gamma \quad\left(A^{3}\right)$ & 5 & $3-15$ & $1-300$ \\
\hline$w * \frac{\text { (chains }}{\mathrm{cm}^{2} \text { ) }}$ & $1 \times 10^{13}$ & $0.5-2.5 \times 10^{13}$ & $1 \times 10^{11}-5 \times 10^{1}$ \\
\hline \multicolumn{4}{|c|}{ *arbitrary in theoretical model } \\
\hline \multicolumn{4}{|c|}{$\begin{array}{l}\text { Although some problems undoubtedly exist in the theoretical model } \\
\text { and must be modified, refined or explained by further experimental } \\
\text { evidence, the results are by far the best representation to } \\
\text { date of a microscopic failure criterion. }\end{array}$} \\
\hline
\end{tabular}




\subsection{OTHER TEMPERATURES AND MATERIALS}

In section 5.3 , the importance of the "effective or apparent" distribution in the "critical flaw" region was indicated. It is the purpose of this section to examine in detail the meaning of these experimental observations especially in relationship to other temperatures, materials and the theoretical model. Further comparison of the experimental stress, strain, and bond rupture data with theoretical predictions at other temperatures and other materials is also discussed. With the apparent success of the theory in predicting room temperature results, it was important to investigate its applicability to other temperatures and even other highly oriented fiber materials. This was not an attempt to do an in depth study, but rather an effort to access the trends and gain insight into the limitations and capabilities of the theoretical model and fracture criterion.

Kinetic rate theory in essence says that the rate at which bonds fracture depends on three main factors, the activation barrier $U_{0}$, the thermal environment $T$, and the stress on the polymer chain $\sigma_{i}$, i.e.

$$
\frac{d C_{B i}}{d t}=\left\{w_{0} \exp -\left[\frac{\left(U_{0}-r \sigma_{i}\right)}{k T}\right]\right\} C_{u i}
$$

where $\frac{d C_{B_{i}}}{d t}=$ the rate of chain scission in the $i^{\text {th }}$ set and all other

As thermal energy is increased, it essentially aids the rate process in overcoming the activation barrier. Likewise an increase in the stress level on a chemical bond has the same effect. Therefore, one might say that the strength of a chemical bond at low temperature is "stronger" that at high temperature due to thermal effects only. Also, if the effective distribution of chain lengths in the flaw region is broad 
(many unequal effective chain lengths) then the stress will not be equally distributed on the individual chains. This results in some chains being highly stressed causing stress concentrations that aid the process over the activation barrier and effectively "weaken" the bond. For a narrow distribution (many chains of the same effective length) the stress is more uniformly distributed on the individual chains and process is not aided to the same extent as with a broad distribution.

In Figure 25, a relationship between the width of the effective distribution in the "critical flaw" region and the ultimate stress for several types of nylon and various temperatures was established. This relationship for each type of nylon will be examined and comparisons with theoretical predictions studied. Figure 43 shows a replot of the experimental data from Figure 25 for nylon 6 \#2 where the distribution widths were established for $-25^{\circ} \mathrm{C}, \mathrm{RT},+50^{\circ} \mathrm{C},+75^{\circ} \mathrm{C}$ and $+100^{\circ} \mathrm{C}$ from the EPR histograms shown in Figure 22. Using the theoretical model parameters established in the room temperature tests for nylon 6 \#1 (section 5.3) and the experimental distribution widths for nylon 6 \#2, the model was used to predict the maximum stress at the respective temperatures. Figure 43a shows the comparison of theoretical results with experimental observations where the theoretical maximum stress values were obtained at the respective experimental distribution values but assuming the temperature in each case was room temperature. This curve therefore indicates the theoretical strength (for the specified parameters) that a fiber can obtain at room temperature as the distribution in the flaw region becomes broad or narrow. Figure 43b shows the comparison of theoretical and experiment results where the 


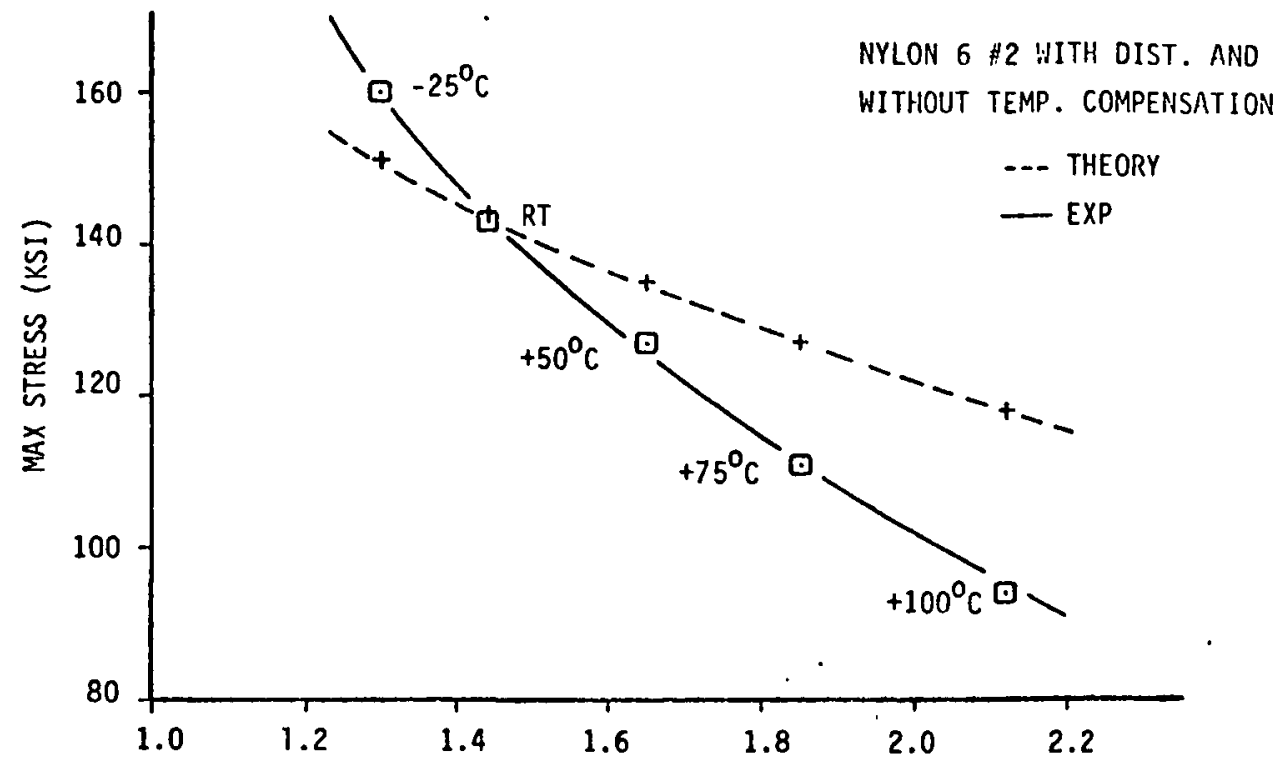

a.

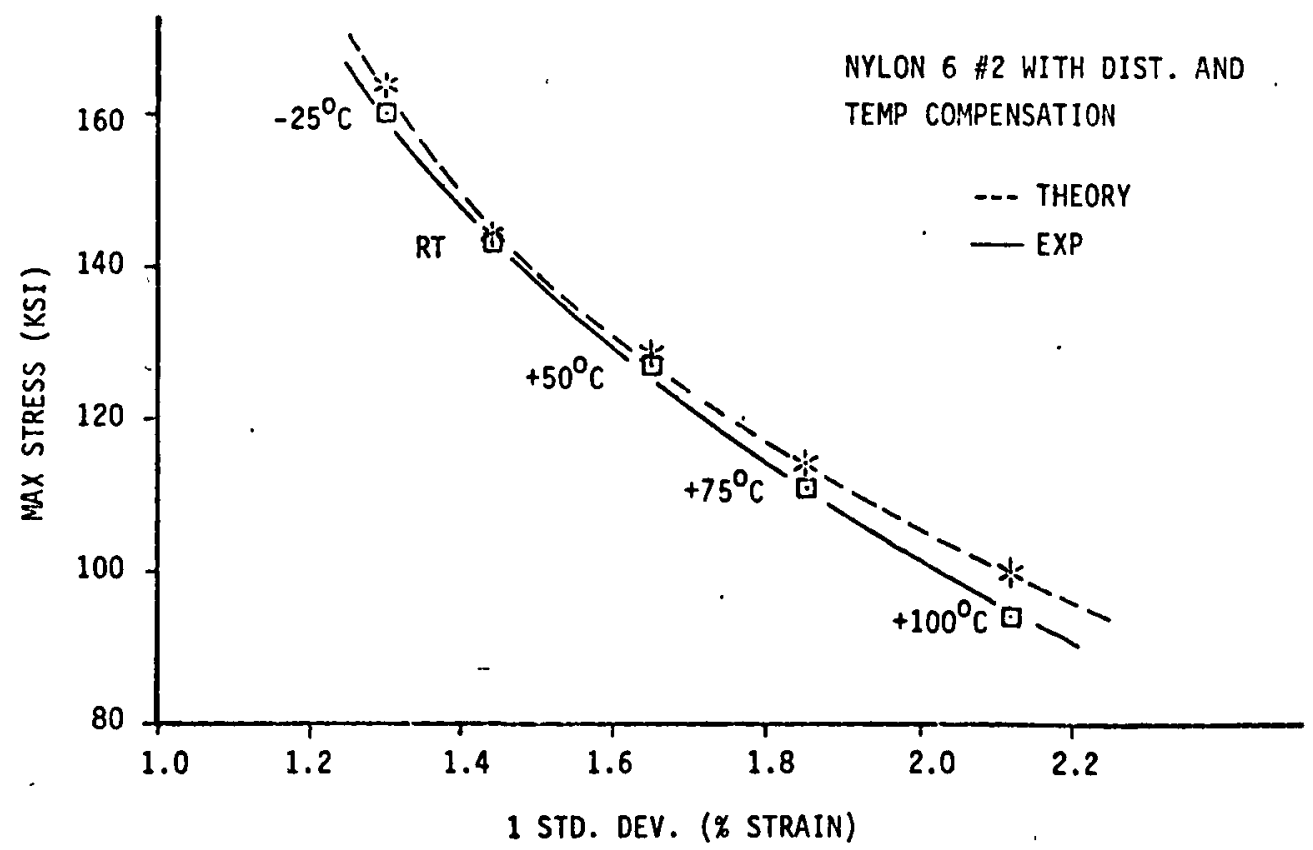

b.

FIGURE 43. Effect of apparent distribution on maximum strength for nylon $6 \# 2$ at several temperatures. 
theoretical maximum stress values were obtained at the respective experimental distribution and temperature values. Comparing theoretical values with corresponding experimental values gives insight into the relationships between atomic occurances, macroscopic observations and the theoretical model. In Figure 43a, the room temperature theoretical and experimental values agree, but comparison of theoretical distribution values with their corresponding experimental values at higher temperatures $\left(+50^{\circ} \mathrm{C},+75^{\circ} \mathrm{C},+100^{\circ} \mathrm{C}\right)$ indicates theoretical maximum stress values which exceed the experimentally observed stress values. This difference of values becomes greater as the temperature increases. This effect can best be explained by noting that the theoretical chemical bonds in this case were assumed to have room temperature "strength" which would make them "stronger" than the high temperature bonds. Therefore, even though the effective distribution became more broad, which lowered the maximum stress, that effect alone was not sufficient to explain the experimental stress values. The opposite effect at the experimental $-25^{\circ} \mathrm{C}$ value is observed in Figure 43a where the theoretical bond is now "weaker" than the experimental bond due to lack of compensation for the change from room temperature to $-25^{\circ} \mathrm{C}$ in the theory. In Figure $43 \mathrm{~b}$ both temperature and distribution values corresponding to the respective experimental values were used in the theoretical model. Note at the higher temperatures $\left(+75^{\circ} \mathrm{C}\right.$ and $\left.+100^{\circ} \mathrm{C}\right)$, the theoretical strength exceeds the experimentally observed strength. Although this difference is not too large, (less than $10 \mathrm{ksi}$ at $\left.+100^{\circ} \mathrm{C}\right)$, it is significant. At high temperatures, Backman (43) has reported results for bulk nylon 66 that indicate 
above $+50^{\circ} \mathrm{C}$ the chains in the amorphous regions appear to "unzip" or pull out rather than fracture. His observations are based on EPR results obtained during slicing and grinding experiments at various temperatures. He attributes the smaller EPR values obtained above $+50^{\circ} \mathrm{C}$ to less backbone chain scission. In the nylon 6 fibers tested by the author, it is expected that as the temperature is increased, some of the tie molecules in the flaw region will reach a "crossover" point where they would rather pullout or "unzip" than fracture. This point varies, of course, with the length of the chain that is buried in the "cry-blk" region. However, at the point where the chain just begins to pull out, the strength of the chain is only slightly less than that required for chain scission. In essence, then, the slightly lower strength at the higher temperatures may be attributed to fewer tie molecules due to some "unzipping" or pulling out. More will be said on this later when the total number of free radicals observed at different temperatures is discussed. Figure 44 shows the effect of considering only temperature compensation without distribution compensation for the same nylon 6 \#2 tests shown in Figure 43. In this representation, the maximum stress of nylon 6 \#2 is plotted as a function of temperature. In Figure 44a the theoretical values corresponding to each temperature were predicted from the model without including the experimentally observed effective distribution changes with temperature. All values were calculated using the distribution observed at room temperature. At temperatures higher than room temperature, the theory predicts maximum stresses greater than those experimentally observed. This is due to the stresses being more uniformly distributed than they would have been if the broader 


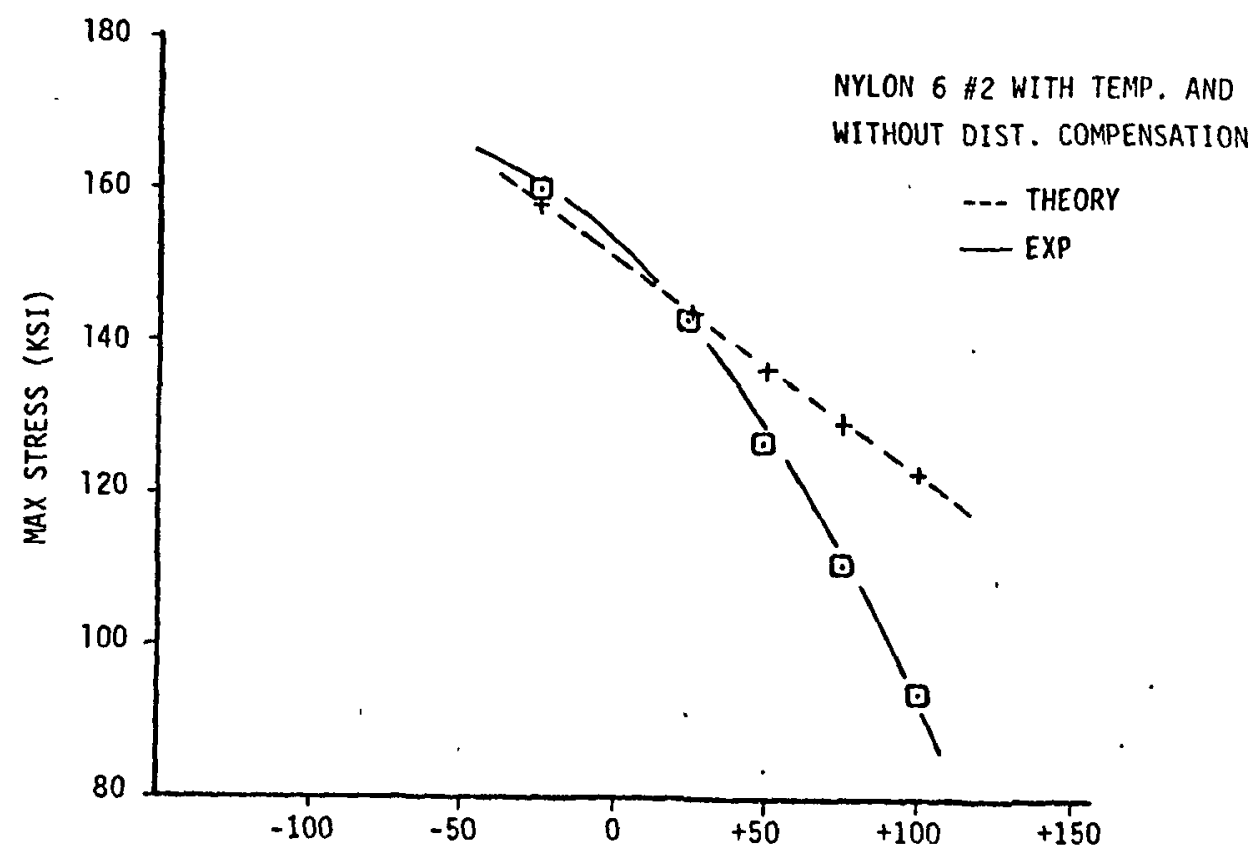

a.

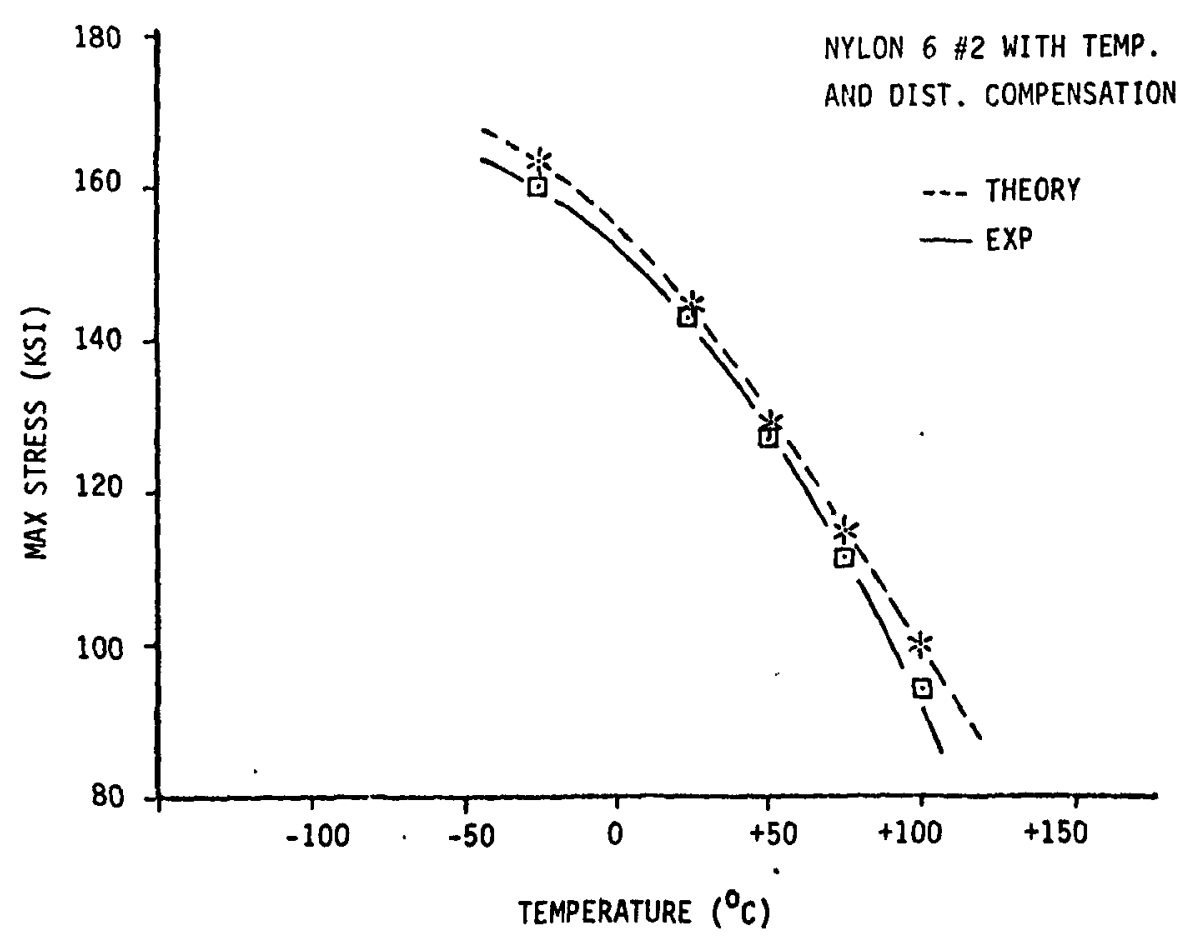

b.

FIGURE 44. Effect of temperature on maximum strength for nylon 6 \#2. 
experimental distribution values observed at the higher temperatures had been used. Even so, the theoretical maximum stress decreases due to a "weakening " of the bond by an increase in temperature. The opposite effect is observed when comparing the $-25^{\circ} \mathrm{C}$ values. In Figure 44b, the correspondence between theory and experiment is shown when both temperature and distribution effects are taken into consideration. The same treatment of the data as explained in Figures 43 and 44 is presented for nylon $6 \# 1$ in Figures 45 and 46 . Here again the same relationships are confirmed.

The correlations are most pleasing and indicate the importance of a failure theory which accounts for two major effects on an atomic scale in order to predict macroscopic behavior. Both effects are the result of the thermal environment but one might be considered an intramolecular effect while the other is an intermolecular effect. The intramolecular effect is the "strengthening" or weakening" of the atomic bond in a single chain due to the decrease or increase in thermal vibrations of the atoms as a result of temperature changes. The intermolecular effect is the tightening or loosening of the interchain forces (secondary bonding) in a thermal environment which in turn determines the allowable slippage, twisting, or uncoiling of the polymer chains. The degree of this tightening or loosening of the microstructure then determines the "effective or apparent" distribution of chain lengths in the flaw regions. The distribution determines the stress concentrations on the individial chains which then determines the degree of weakening of the bond due to this stress. In essence, then, even though viscoelastic effects as a function of time are not included, the distribution tends to account for some of these effects as a 


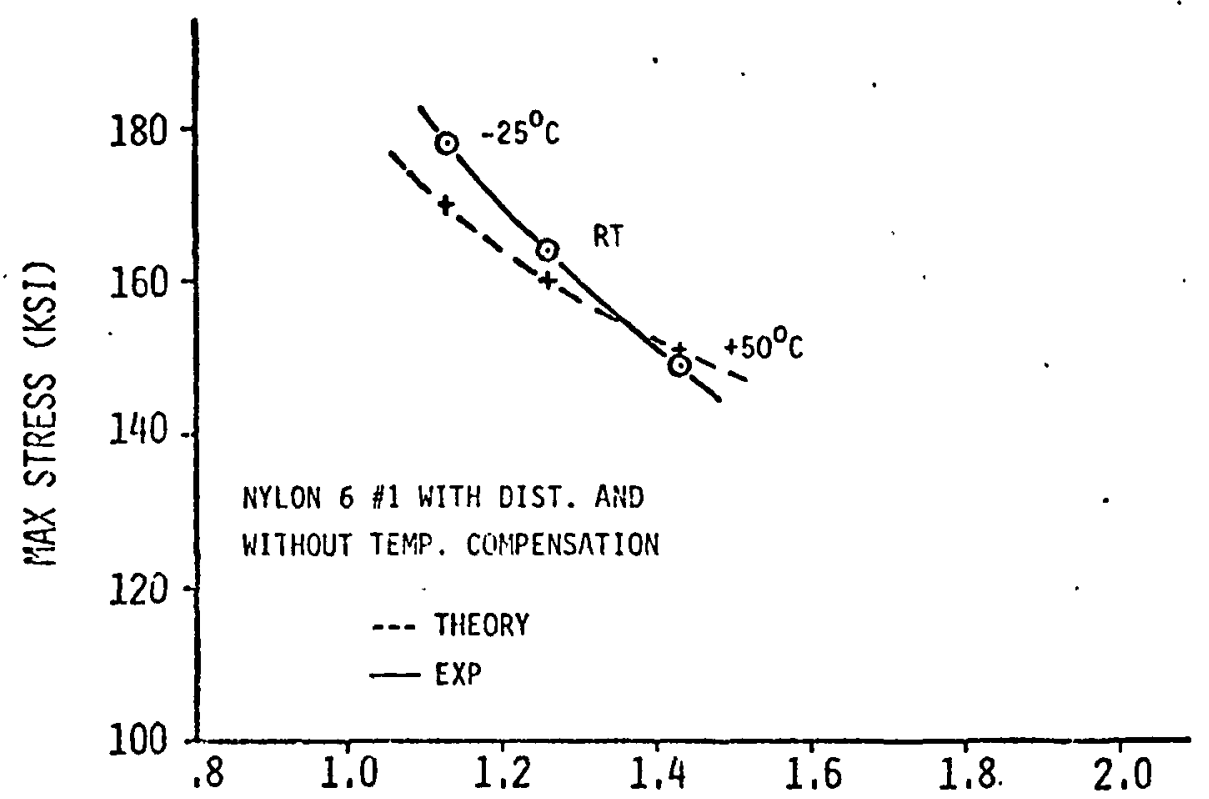

a.

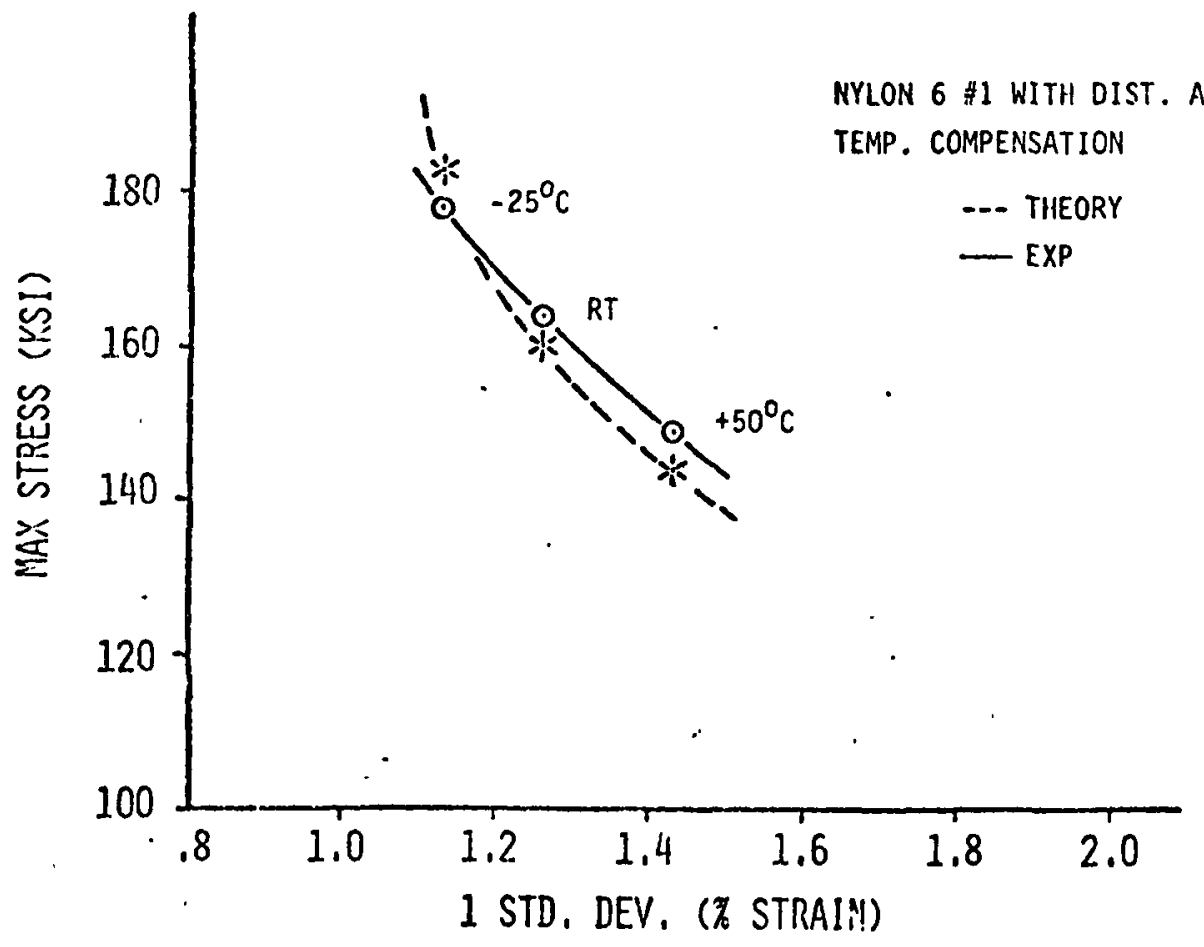

b.

FIGURE 45. Effect of apparent distribution on maximum strength of nylon $6 \# 1$ at several temperatures. 

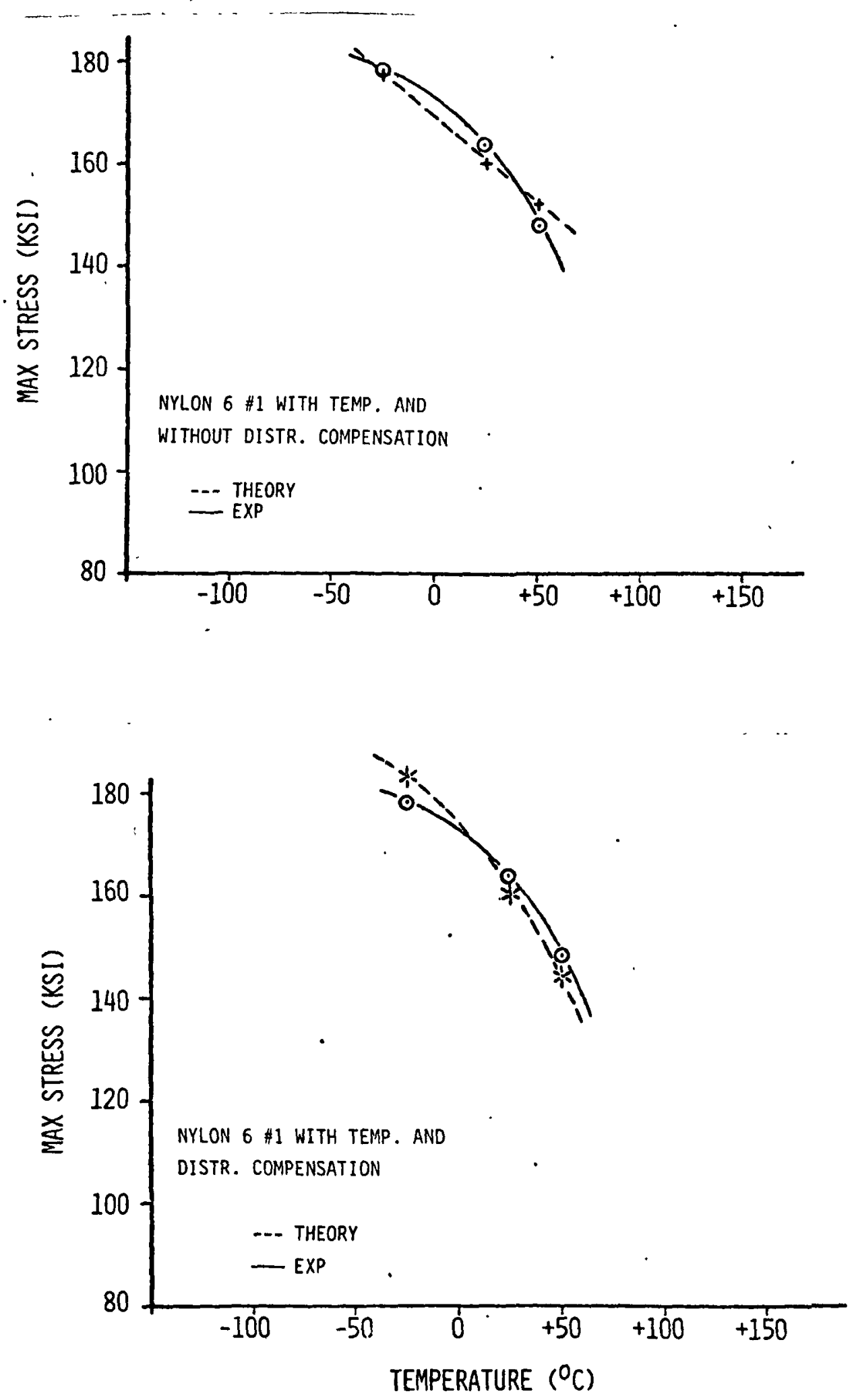

FIGURE 46. Effect of temperature on maximum strength for nylon $6 \# 1$. 
function of temperature. Another important point to consider from the above results is that the two types of nylon 6 samples appear to be very much alike with the exception of a difference in the apparent distribution of the critical flaw regions. The same parameters which were determined as the "best fit" values for nylon 6 \#1 also were successful in predicting the behavior of nylon 6 \#2 if the experimentally determined distribution values for the corresponding temperatures and nylon were used. Nylon 6 \#l exhibited a more narrow distribution at room temperature than did nylon $6 \# 2$ and therefore nylon 6 \#1 was stronger and yet more brittle than nylon 6 \#2 at the same temperature. The ratio of the original lengths of the "cry-blk" to "critical flaw" region; the ratio of number of chains in the "cry-blk" to "critical flaw"; the kinetic rate constants, and the total number of tie chains in the flaw region all remained the same in the theoretical predictions for both nylons and all temperatures. As the same EPR spectra was observed for both nylon $6 \# 1$ and nylon 6 \#2 and both have the same chemical constituents, the rate constants should truly be constants and only structure changes should be necessary to predict the experimentally observed results. This in fact was the case for these two nylons. Another important factor, i.e. the total number of tie chains in the flaw region will be discussed more as we proceed with the theoretical and experimental comparisons. The importance of considering this parameter was indicated in the results of the high temperature nylon 6 \#2 tests of Figure 43b. Figure 47 , is a comparison of theoretical and experimental stress, strain and bond rupture data for nylon 6 \#1 from constant strain rate tests. Two different strain rates at each of three temperatures $\left(-25^{\circ} \mathrm{C}, \mathrm{RT},+50^{\circ} \mathrm{C}\right)$ were theoretically 

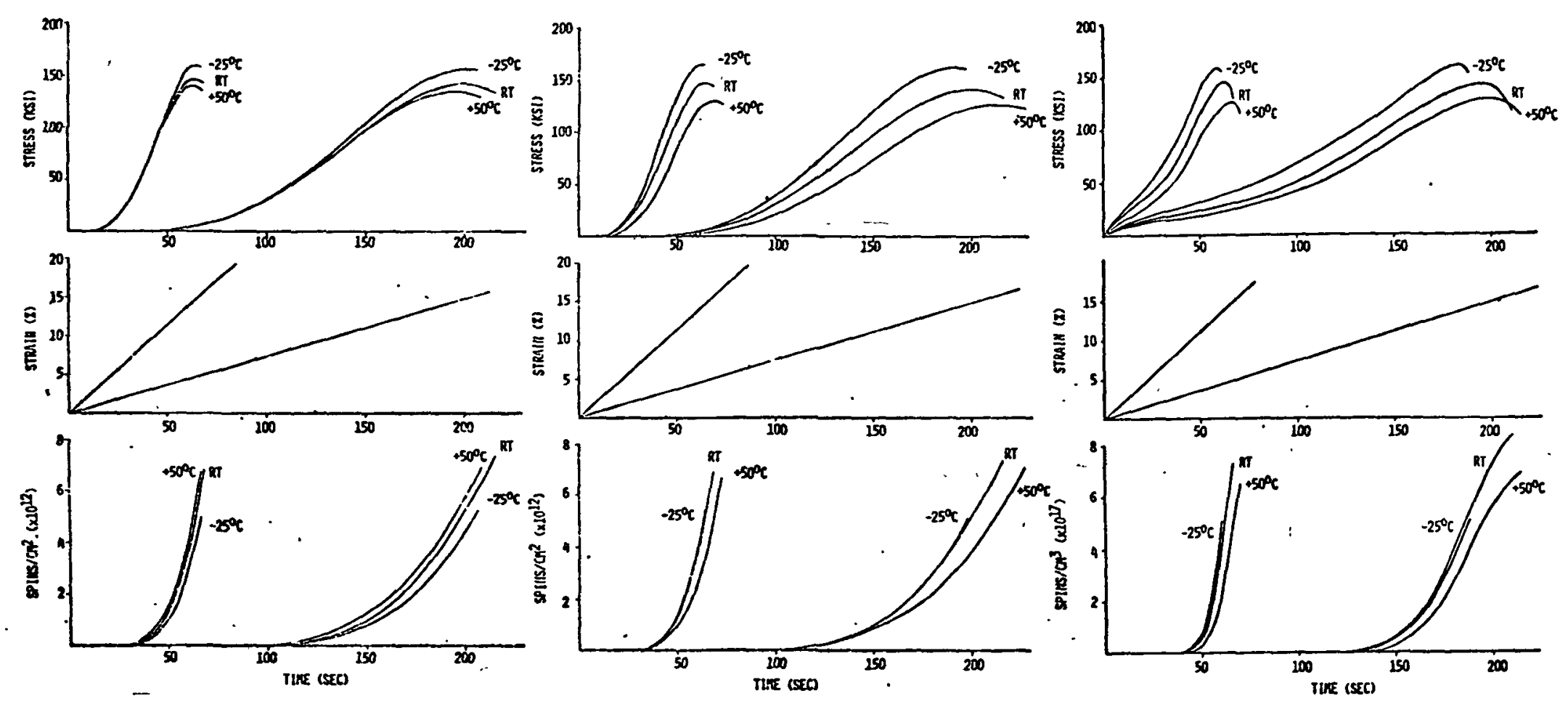

a. Theoretical - Temperature compensation only.

b. Theoretical - Temperature and distribution compensation.

c. Experimental.

FIGURE 47. Constant.strain rate experimental and theoretical predictions for nylon 6 \# at $-25^{\circ} \mathrm{C}$, room temperature, and $+50^{\circ} \mathrm{C}$. 
predicted and experimentally observed. Several very important observations can be made from this data. If we consider again the rate equation that was selected as the criterion for predicting chain scission (Equation. 33) we have:

$$
\frac{d C_{B i}}{d t}=\left\{w_{0} \exp \left[-\frac{\left(U_{0}-\gamma \sigma_{j}\right)}{k T}\right]\right\} C_{u i}
$$

Considering only changes in temperature $(T)$, Equation 33 indicates that at low temperatures the rate of chain scission should be less than at high temperatures. Therefore, if the effective distribution does not change with temperature, the EPR curve for $-25^{\circ} \mathrm{C}$ should shift to later times on the time axis relative to the room temperature curve and the $+50^{\circ} \mathrm{C}$ EPR curve should shift to earlier times. Figure 47a shown this relationship as predicted theoretically by the model for $-25^{\circ} \mathrm{C}$, RT and $+50^{\circ} \mathrm{C}$ where the effective distribution was assumed to remain unchanged from the experimentally observed room temperature value. Note also in Figure 47 a that the stress peaks occur in magnitude and order as $+50^{\circ} \mathrm{C}, \mathrm{RT}$ and $-25^{\circ} \mathrm{C}$, where $+50^{\circ} \mathrm{C}$ occurs first in time and lowest in maximum stress. Comparing the predictions of Figure 47 a with the experimental observations shown in Figure $47 \mathrm{c}$, we note the order of the predicted and experimental curves are reversed with respect to each other in both the stress peak time sequence and the EPR curve time sequence. Therefore, we can conclude that not only would the Zhurkov Equation (Equation 3) fail to predict these experimental temperature test results but so also would the proposed theory (based on a distribution of stresses) fail to predict temperature tests if no compensation for a changing distribution with temperature was included in the model. As the effective 
distribution changes with temperature, it could shift in several ways, two of which would be about its mean value or about one end. Figure 48 shows the effect that these two types of distribution shift would have on the relative position of the EPR chain scission curves for three temperatures. In Figure $48 \mathrm{a}$, the relative position of the curves is shown where the effective distribution is assumed to not change with temperature. Figure 48b shows the relative positions where the effective distribution is changed about the mean value and $48 \mathrm{c}$ shows the relative values of the change occurs about the minimum length end. Comparing these results to the experimental observations in Figure $47 \mathrm{c}$ shows that the effective distribution changes with temperature by shifting about the minimum chain length in the critical flaw region. Figure $47 \mathrm{~b}$ indicates the results of the theoretical predictions when the effective distribution is assumed to shift about the minimum length and as the temperature is changed. This can be visualized by noting that in the flaw regions the chains all have some original structure which undoubtedly includes kinks, coils, folds or other non-uniform length configurations. The shortest chains in this group are most likely extended taut between the two adjacent "cry-b7k" regions. As the sample is loaded and the region become extended, each chain assumes some effective end to end length according to the effects of temperature and/ or strain rate on the mobility of the microstructure. Therefore, at low temperatures, the chains shift to shorter effective lengths resulting in a shift of the distribution mean toward the minimum chain length side of the distribution. Therefore, the changing distribution and changing temperature have opposite effects on the position of the chain scission curves relative to the time axis (a two fold mechanism) and the macrosopically observed experimental EPR chain scission results are 

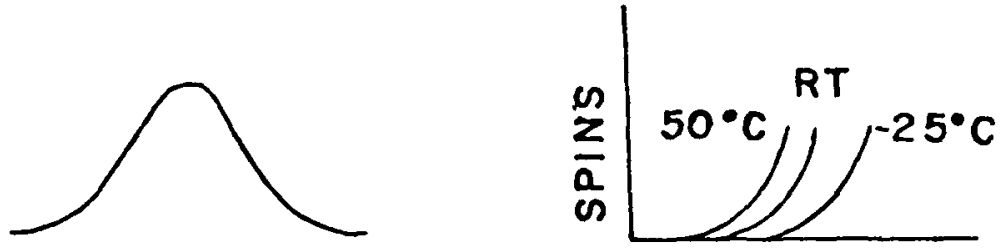

TIME

a. No distribution change with temperature changes.
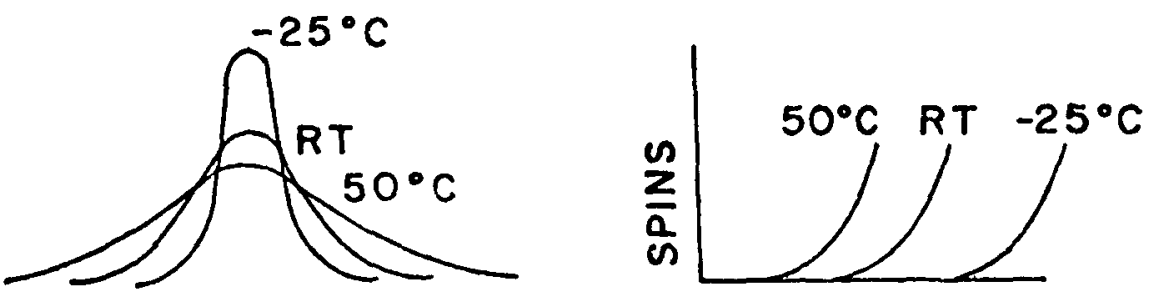

TIME

b. Distribution shifts about mean value as temperature changes.
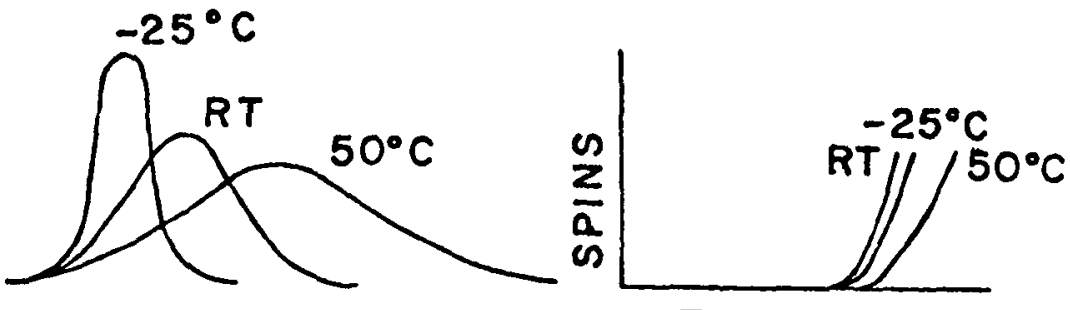

TIME

c. Distribution shifts about minimum length end as temperature changes.

FIGURE 48. Schematic representation of relative EPR results with respect to two types of distribution changes. 
a compromise of the relative effects of both processes. Another observation from the data shown in Figure 47 is the relationship (or lack of relationship) between the total number of tie chains in the flaw region and the number of tie chains observed at the catastrophic fracture point. The theoretical fracture point in each case was determined from the experimental results. The point occurred at a specific time increment beyond the peak or maximum stress for each test. The number of spins (two spins per bond) at the fracture point and at the maximum or peak stress point for nylon 6 \#1 are plotted as a function of temperature in Figure 49a and $b$ respectively. The theoretical values in Figure 49 were plotted from the corresponding predictions in Figure $47 \mathrm{~b}$. Several observations can be made from the comparisons. The EPR values recorded at fracture are dependent on how far beyond the maximum stress point the test proceeds before catastrophic failure occurs. As the EPR curve at this point appears almost exponential for constant strain rate tests (Figure 47), small time differences in the catastrophic fracture point can make large differences in the total number of chain scissions observed by the EPR spectrometer. In fact, the more narrow the distribution the sooner catastrophic failure will generally take place and therefore EPR results based on the fracture point may appear significantly smaller. For instance, comparing the differences between the experimental spin values at $-25^{\circ} \mathrm{C}$ and room temperature for the fracture point values of Figure $49 \mathrm{a}$ and the maximum stress values of Figure $49 \mathrm{~b}$ indicates a thirty per cent increase in relative spin values at the fracture point compared to the values at the maximum stress point. This increase 


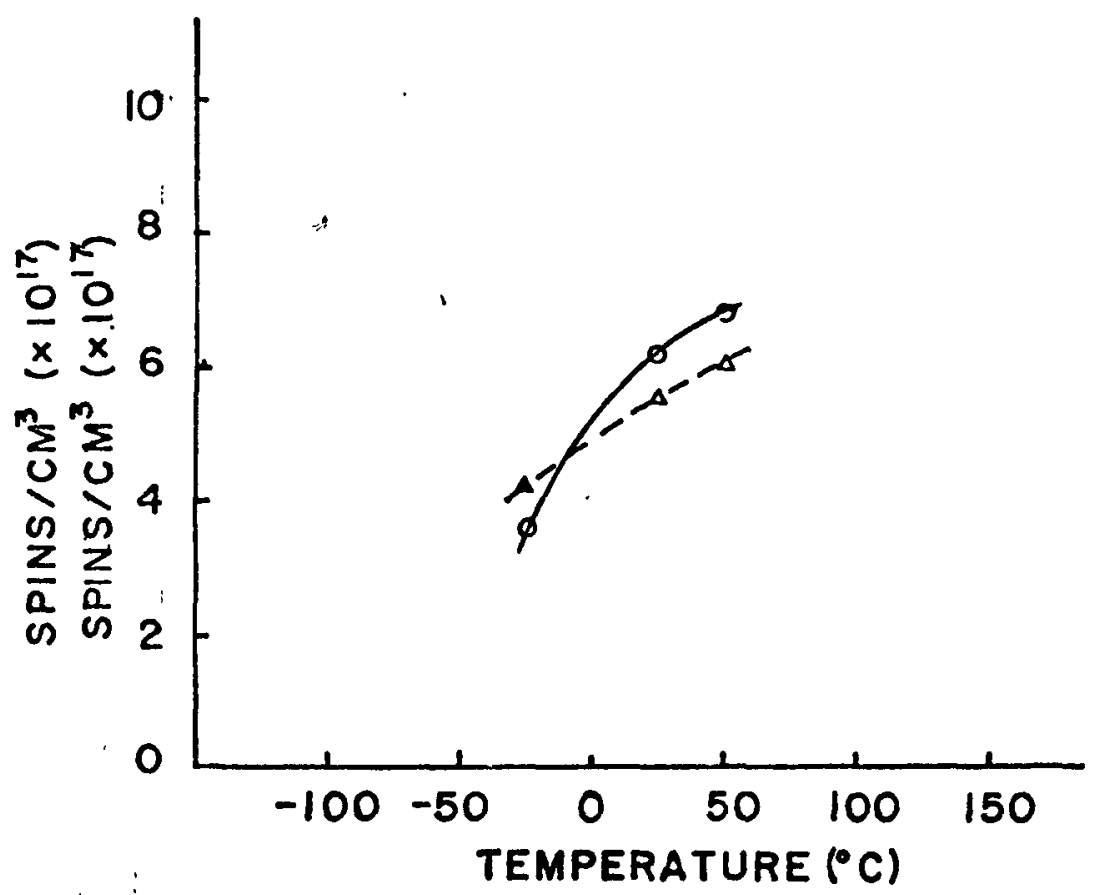

a. Fracture Point

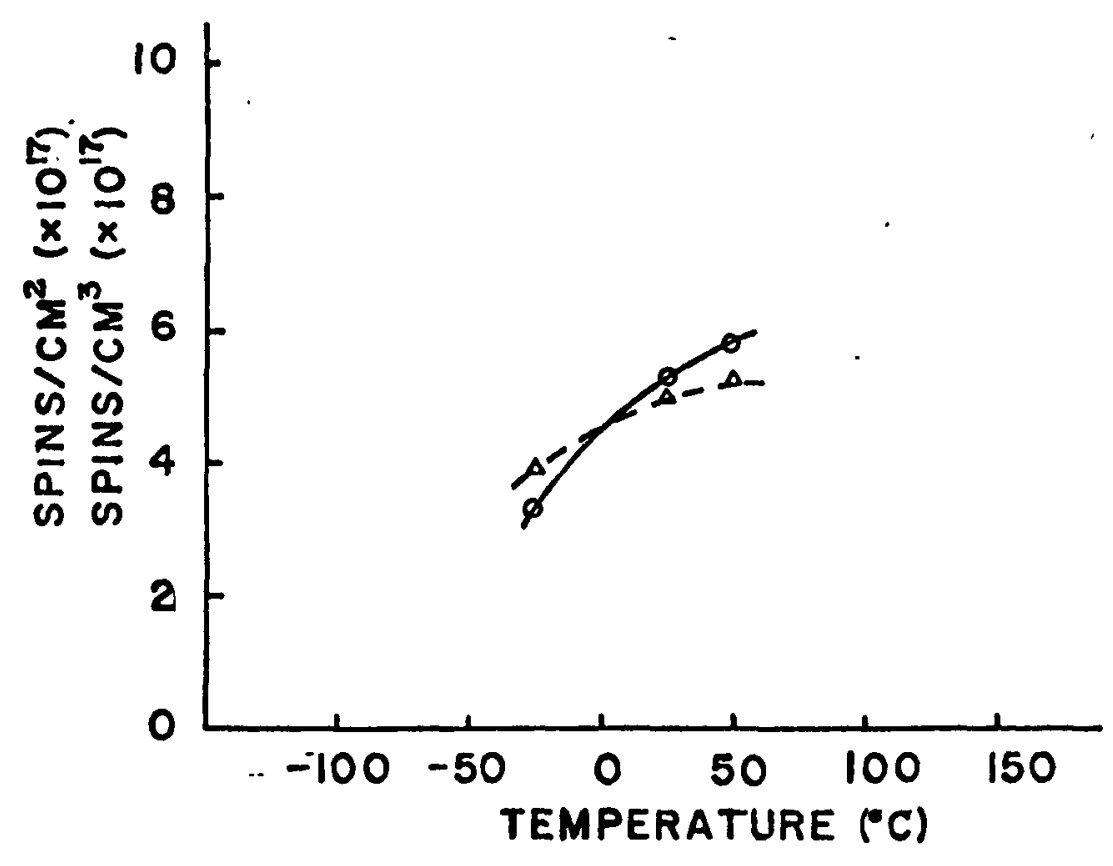

b. Maximum Stress Point

FIGURE 49. Effect of temperature on accumulative chain scissions at fracture and at maximum stress pqint for nylon 6 \#1. (Theory Spins $/ \mathrm{cm}^{2}$ ). 
can be attributed to differences in the time increment to fracture after the maximum stress point was reached. Another interesting observation can be made from Figure 49b. Note that the experimental number of chain scissions increases with increasing temperature. This increase is also noted in Figure 49a and at first glance might be attributed to small differences in the fracture point which could result in the large spin value variations. However, apparently only a portion of this increase can be attributed to such a process, as the results in Figure $49 \mathrm{~b}$ also show this increasing trend. Although the reason for this trend is not completely understood, it is important to note that the theoretical model also predicts an increase in chain scissions at the maximum stress point as the temperature increases. This theoretical relationship is obtained even though the total number of tie molecules in the theoretical flaw region remained constant. The differences in the experimental and theoretical values are most likely related to the lack of including some viscoelastic effects as discussed in Section 5.3. It is therefore concluded from these observations that the total number of tie molecules in this particular nylon 6 fiber have not substantially changed during tests in this temperature range $\left(-25^{\circ} \mathrm{C}, \mathrm{RT}\right.$, and $\left.+50^{\circ} \mathrm{C}\right)$. At higher temperatures, such tie molecule changes might be expected as was discussed earlier in this section. Thus, even though the total number of tie molecules in the flaw regions may remain constant, the number observed by EPR at fracture or at maximum stress may vary depending on the distribution, temperature, strain rate, or other similar factors.

Additional strain rate tests for nylon 6 \#2 were experimentally and theoretically compared at three temperatures, $-25^{\circ} \mathrm{C}, \mathrm{RT}$, and $+50^{\circ} \mathrm{C}$. 
The results from these tests were very similar to those for nylon 6 \#1 and substantiated the necessity for both temperature and distribution compensation. These results are shown in Figure $50 \mathrm{a}, \mathrm{b}$, and $\mathrm{c}$, where Figure $50 \mathrm{a}$ is the theoretical predictions with temperature compensation only, Figure 50b is the theoretical predictions with both temperature and effective distribution compensation, and Figure $50 \mathrm{c}$ is the experimental results. Likewise, Figure 51 is the theoretical and experimental data from Figure 50 relating chain scission at the fracture point and the maximum stress point to the corresponding test temperature $\left(-25^{\circ} \mathrm{C}\right.$ RT and $+50^{\circ} \mathrm{C}$ ). Note in Figure $51 \mathrm{a}$, that the fracture point spins at room temperature exceed those at $+50^{\circ} \mathrm{C}$, however, the maximum stress point values of Figure 51b show the opposite trend. This apparent contradiction results from a room temperature test that exhibited a longer than usual time increment beyond the maximum stress point before failing catastrophically (see Figure 50c). Note how this additional small time increment effected the fracture spin data. From these observation, the author notes that caution should be exercised in comparing EPR fracture data at different temperatures or between materials with different effective distributions. Four additional points are noted on Figure 51 at higher temperature values $\left(+75^{\circ} \mathrm{C}\right.$ and $\left.+100^{\circ} \mathrm{C}\right)$. The experimental points 1 and 2 shown in Figure 51 a were obtained from step strain data (no strain rate tests were run at these temperatures). The apparently smaller total spin values at these temperatures merits some interesting future research. Are the small values due to decay of the spins at these high temperatures or are less chain scissions occuring due to "pull out" or "unzipping" as previously discussed in conjunction with Figure 43b? Decay of free radicals at temperatures 

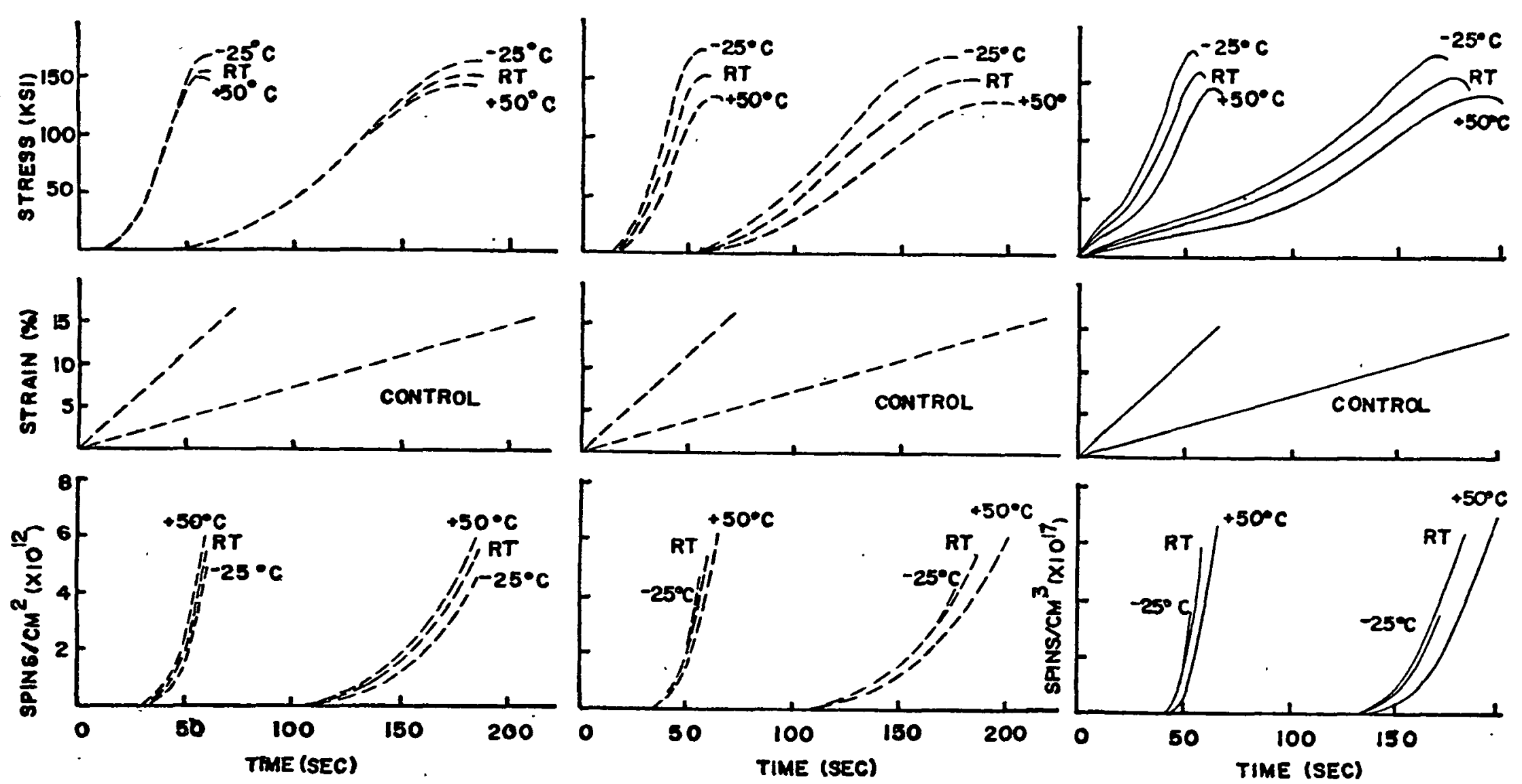

a. Theoretical--temperature
compensation only

b. Theoretical--temperature and distribution compen-

c. Experimental sation

FIGURE 50. Constant strain rate experiment and theoretical predictions for nylon $6 \# 2$ at $-25^{\circ} \mathrm{C}$, room temperature, and $+500 \mathrm{C}$ (theory--spins $/ \mathrm{cm}^{2}$ ) 


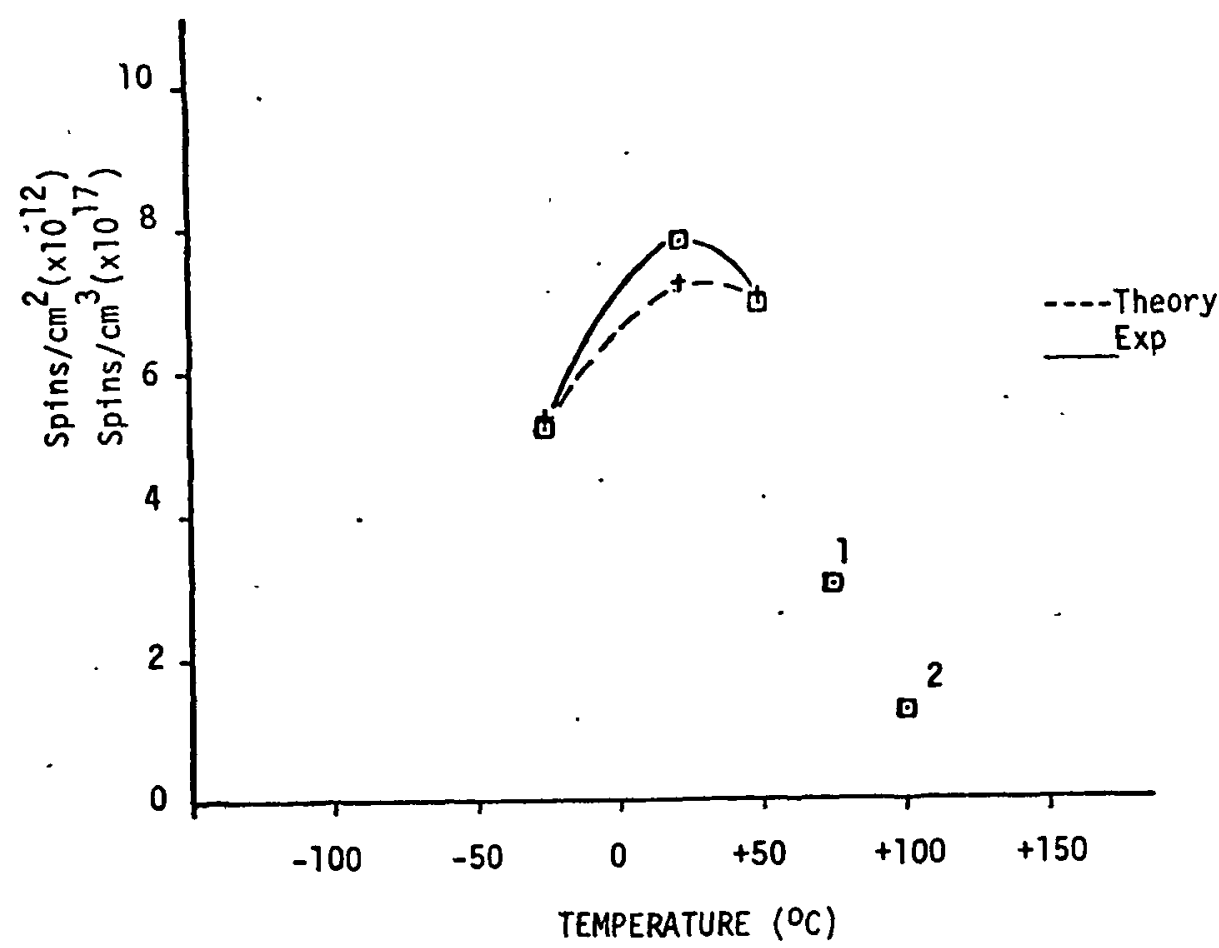

a. Fracture Point

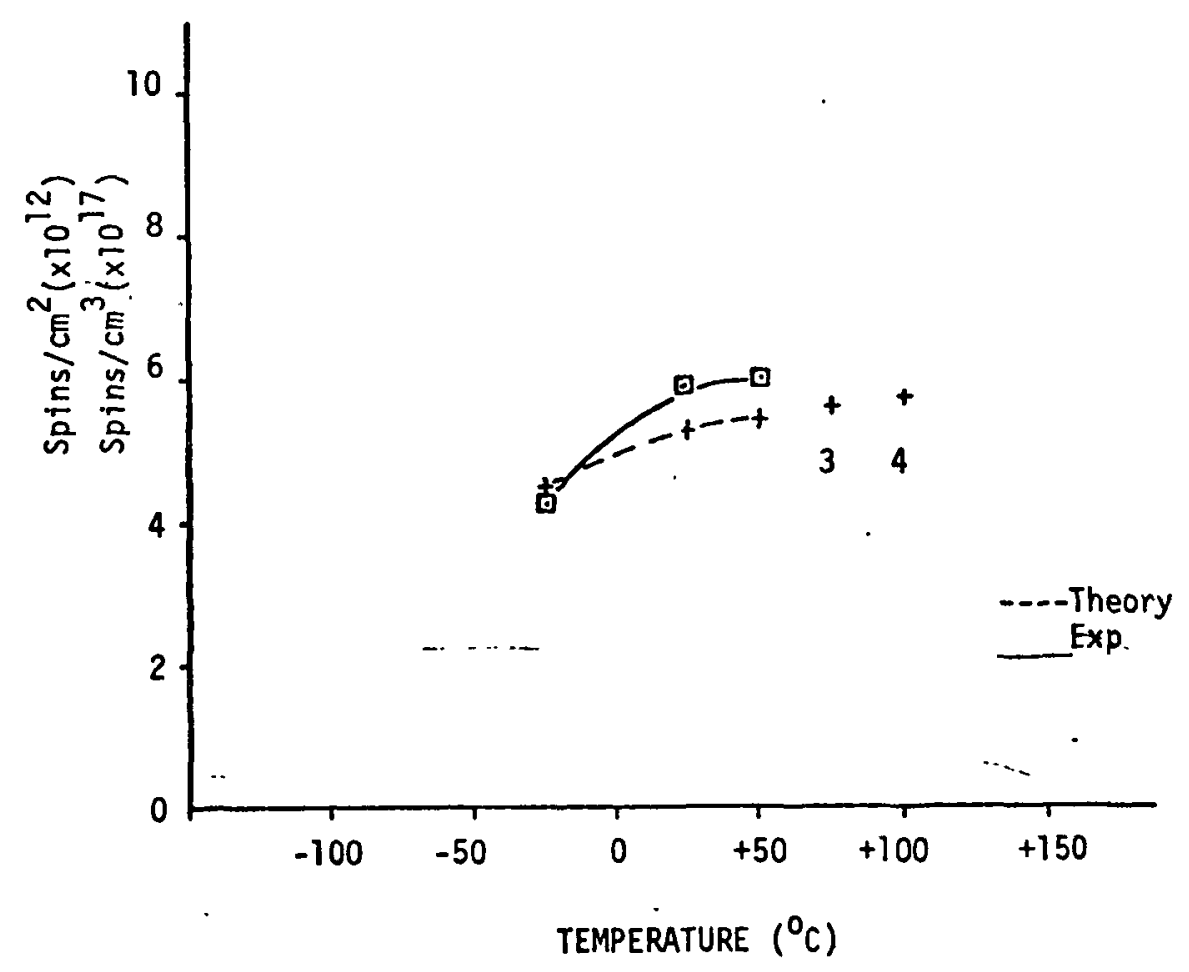

b. Maximum Stress Point

FIGURE 51. Effect of temperature on accumulative chain scissions at fracture and at maximum stress point for nylon 6 \#2. (Theory - Spins $/ \mathrm{cm}^{2}$ ). 
below $+50^{\circ} \mathrm{C}$ in a helium environment was observed to be small or negligible for the time span necessary to complete the constant strain rate tests. However, at temperatures above $+50^{\circ} \mathrm{C}$, decay is a factor and detailed studies of its effect need to be conducted before this question can be answered. Points 3 and 4 in Figure 51b are from theoretical strain rate tests and show that if the total tie molecules in the flaw region remain a constant, then the portion observed at the maximum stress point should slowly increase with temperature. It is the author's opinion from these observations and the results of Figure 43b, that some chain pull out or "unzipping" does begin to occur at temperatures above $+50^{\circ} \mathrm{C}$, effectively reducing the total number of tie molecules in the flaw region. This is in agreement with results of Backman (43) as previously discussed.

Another interesting facet of the current study was the theoretical maximum strength that might be obtained from a nylon 6 fiber characterized at room temperature by a specific set of parameters, if only the width of the effective distribution was varied. Such a theoretical curve is plotted in Figure 52 using the "best fit" parameters, and varying only the effective distribution width. This particular curve is for a "cry-blk" to flaw region chain number ratio (RC) of 30 . The maximum stress values for a particular distribution width can be observed from this curve. Note that nylon 6 \#1 and nylon 6 \#2 fibers both fall.on this curve at room temperature but at different distribution width values. This reinforces previous observations of the basic similarities between the two nylon fibers with the exception of the "as received" distribution uniformity. Also, note that the nylon 6 \#2 annealed fiber and the nylon 66 fiber do not fall on the "best fit" 


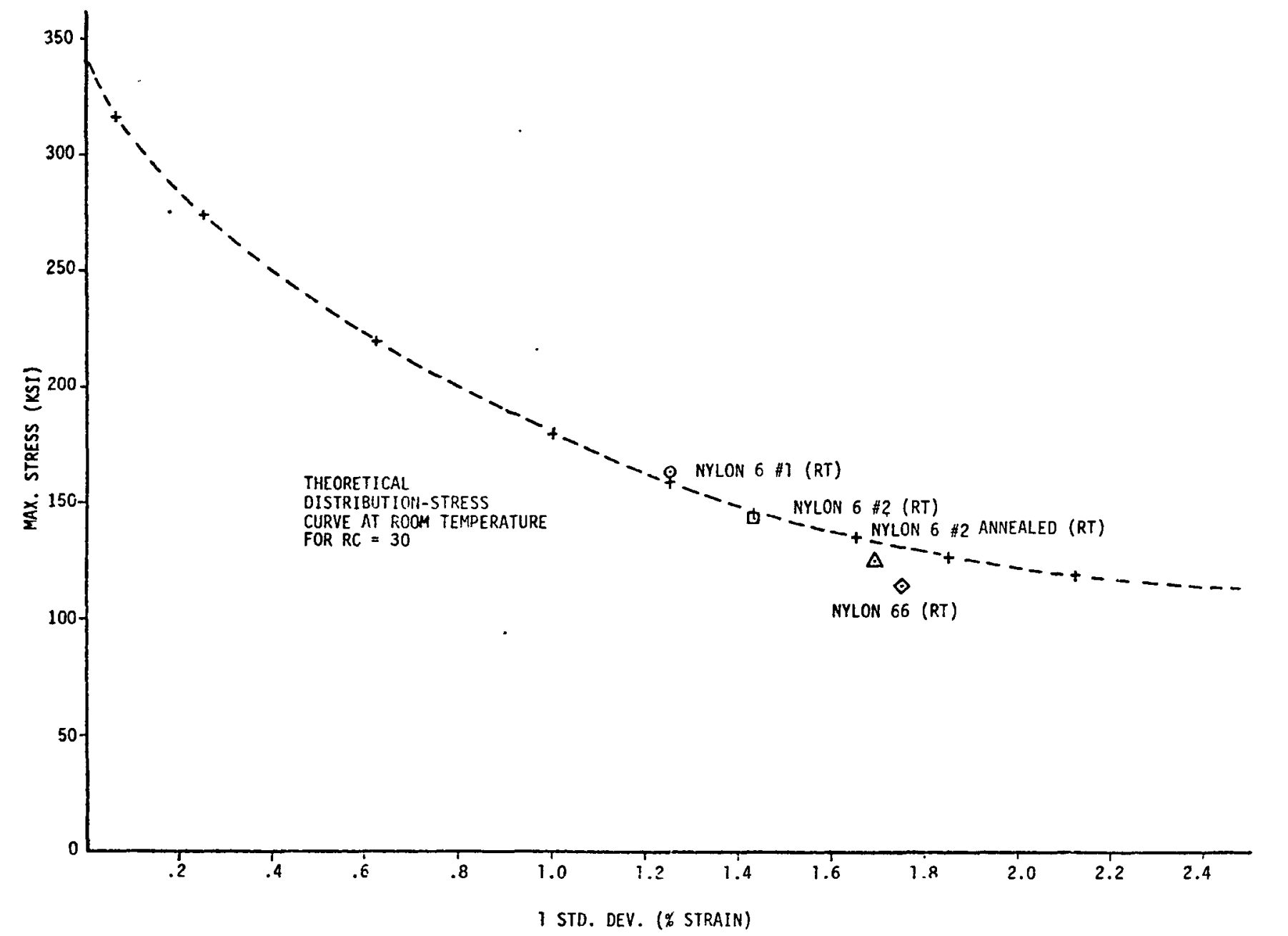

FIGURE 52. Theoretical maximum stress relationship to distribution for one set of specific input parameters. 
parameter room temperature curve indicating some basic differences. The theoretical maximum strength value for a distribution of zero width, i.e. all tie molecules of equal length, is predicted to be approximately $340 \mathrm{ksi}$. Noting that the number of tie molecules is 30 times less than the number that might possibly occur in a perfect structure of infinite chain length, we obtain a maximum possible stress value of $10,200 \mathrm{ksi}$ for a perfect structure. This value is very close to the theoretical value of the strength of a single polymer chain.

An important inquiry about the proposed model and failure criterion is whether the concepts can be extended to materials other than nylon 6. Nylon 66 being similar in chemical composition, but exhibiting many different physical porperties was chosen for preliminary testing and evaluation. The apparent distribution width was measured by step strain tests at room temperature (Figures 24 and 25) and experimental constant strain rate tests conducted at two different rates, also at room temperature. Figure 53 compares the experimental observations . with theoretical predictions from the model for the constant strain rate tests. The theoretical curve labeled $\mathrm{T} 1$ was obtained using the nylon 6 "best fit" parameters with the nylon 66 effective distribution. Two important differences were noted between the theoretical and experimental curves. First, the theoretical strength was too high and second the number of spins or chain scissions at fracture was too great. From these observations and the position of the nylon 66 value on Figure 52, it was concluded that the flaw regions in this particular nylon 66 sample material contained fewer tie molecules than the corresponding nylon 6 materials. Also, the distribution for nylon 66 appeared to be shifted to higher strain values than that 

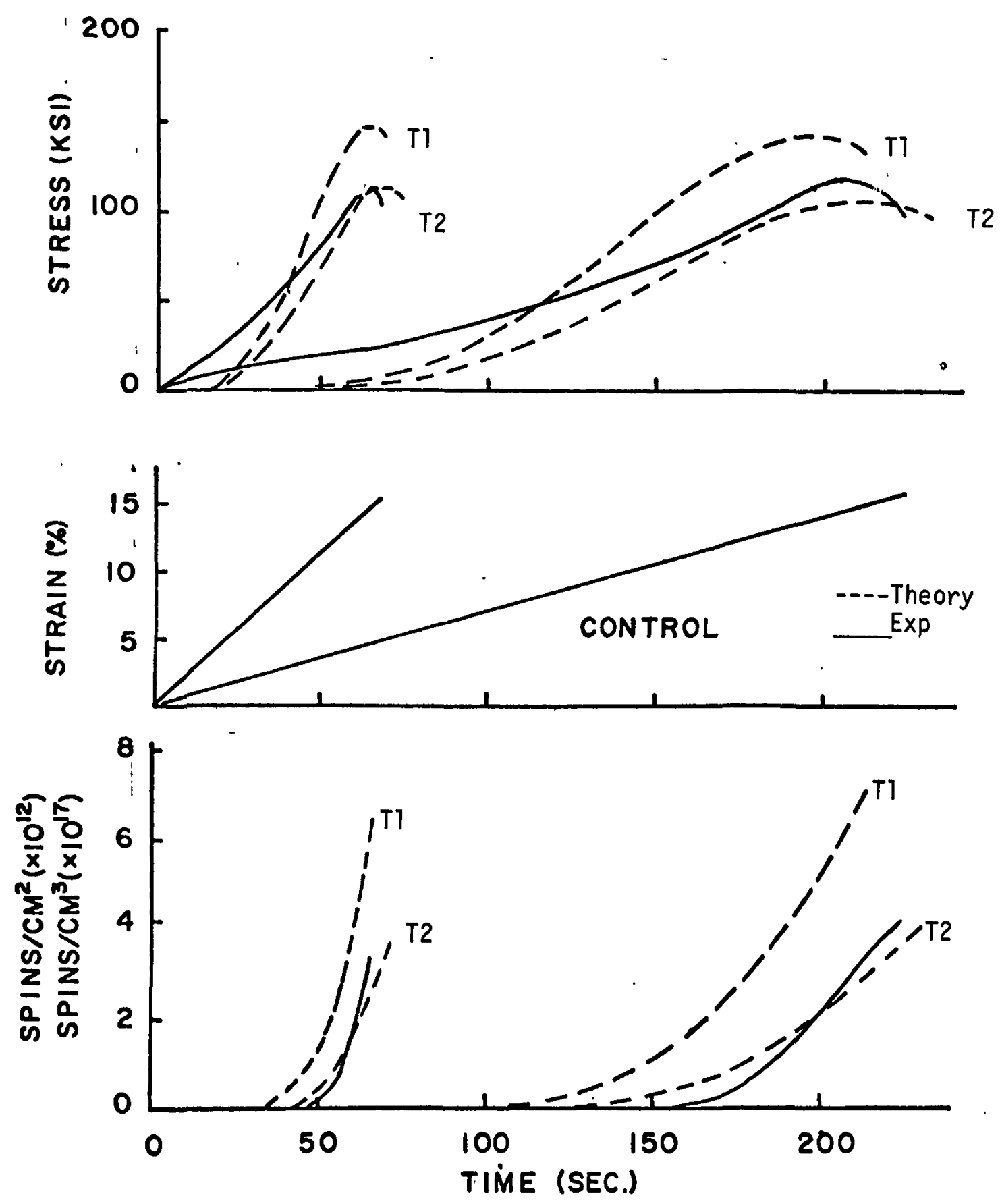

FIGURE 53. Nylon 66 constant strain rate room temperature tests. (Theory - Spins $/ \mathrm{cm}^{2}$ ). 
observed for the nylon 6 samples. Compensation for such effects was made in the model parameters, i.e. the total number of tie molecules in the flaw region was set at $0.6 \times 10^{13}$ chains $/ \mathrm{cm}^{2}$ and the ratio of number of chains in the "cry-blk" to the flaw region was increase to a $\mathrm{RC}$ value of 40 . The relative position of the nylon 66 distribution with respect to the nylon 6 value was noted and the chain length ratio $\mathrm{RL}$ changed to 4.5 . The other model parameters, i.e. the kinetic rate constants $U_{0}, w_{0}$ and $\gamma$ along with the modulus of elasticity $E_{b}$ remained unchanged. These latter values would not be expected to change as the EPR spectra for nylon 66 is the same as nylon 6 most likely indicating chain scission of identical bonds. With the above parameters, the theoretical curve $T 2$ was predicted and compares very well with the experimental data. These results are indeed exciting as they indicate the feasibility of extending the theory to other highly-oriented materials. A further interesting observation is that $X$-ray results from these same two fiber types (23) indicate that more chain folding occurs in the nylon 66 samples. Such folding may account for the larger $\mathrm{RC}$ ratio value and smaller total number of tie molecules in the flaw regions. These factors in turn result in a weaker fiber and less chain scission at fracture.

No additional testing of the slack annealed nylon 6 \#2 fiber was conducted other than the step strain tests which were used to characterize the distribution. However, combining experimental observations of Park (23) obtained from X-ray data and noting the position of this sample in Figure 52, several observations can be made. During slack annealing, there is an increase in chain folding as observed from X-ray data (see Figure 7) (23). This would result in 
in a slight decrease of tie molecules in the flaw regions and a possible large increase in the chain ratio parameter $\mathrm{RC}$, i.e. one tie chain could fold into several effective "cry-blk" chains. Such an effect would account for the experimentally observed lower than "best fit" theoretical values observed in Figure 52, but also would satisfy the total observed chain scission data for the annealed sample, i.e. the total experimentally observed chain scission data for the annealed sample was slightly less than that observed for the control, but not significantly so. Another effect of the slack annealing process is that it apparently redistributes the tie molecules more non-uniformly and perhaps relaxes or replaces the shortest chains in the region by a slightly longer set. These observations results from the apparent broadening of the experimentally observed distribution and the shift of this distribution to higher strain values. Further studies of both slack annealed and tension annealed samples using the proposed theory would be most enlightening.

As a final note, Figure 54 shows the relationship between experimental results and theoretical predictions at room temperature for the three types of nylon tested in this study, i.e. nylon $6 \# 1$, nylon $6 \# 2$ and nyton 66 .

Although these preliminary explorations into other temperatures and materials have been most enlightening, much further work needs to be done with other load inputs, other temperatures, and other or modified materials. It is the author's opinion that further in depth studies will strengthen the theory and provide greater insight into the fracture process. 

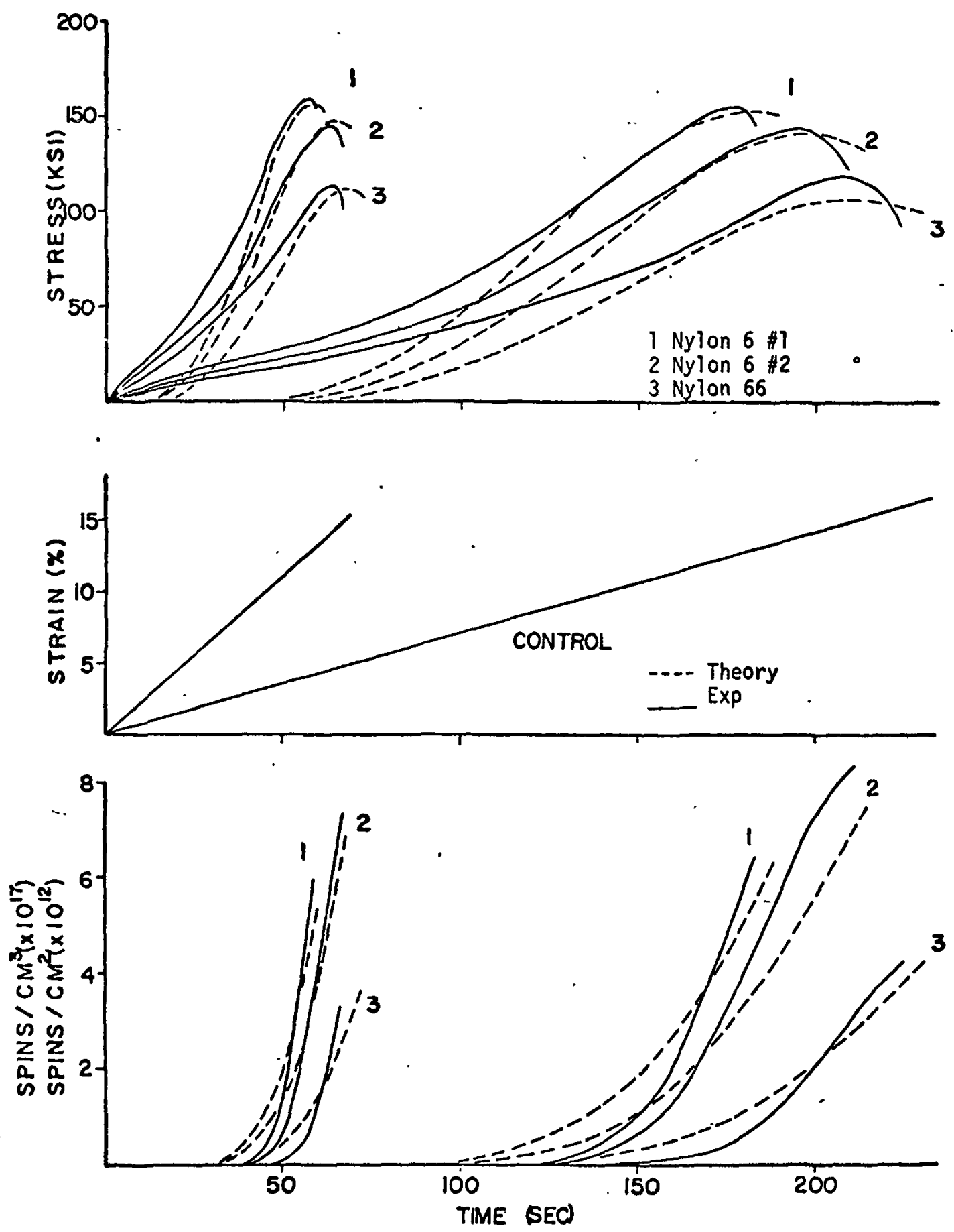

FIGURE 54. Constant strain rate room temperature tests for nylon 6 \#2, and nylon 66. (Theory spins $/ \mathrm{cm}^{2}$ ). 


\subsection{SUMMARY AND CONCLUSIONS}

The results of this study have been presented and discussed in detail in the previous sections. It is the purpose of this section to extract the most important of these observations and present them here in a meaningful manner.

\subsection{RESEARCH OBJECTIVE AND APPROACH}

The goal of this study was to establish a microscopic failure criterion for highly ordered semi-crystalline polymers in fiber form which would relate microscopic occurences to macroscopic behavior. Nylon 6 fibers were chosen to represent the class although some prelinary studies were also conducted on nylon 66 fibers. Electron Paramagnetic Resonance (EPR) techniques and a specially built servocontrolled hydraulic loading system were used to obtain experimental stress, strain bond rupture data from the fiber samples for various input loads. These results were compared, for corresponding input loads, with theoretical data predicted by an idealized mathematical model which incorporated a specific failure criterion. The model selected assumed that the fiber microstructure could be represented by a "sandwich" structure of alternating disordered and highly-oriented regions along the fiber axis. One representative unit was chosen and characterized in terms of the suspected failure process. The representative unit was divided into two regions, one of which was a disordered "weak link" or "critical flaw" region consisting of tie chain molecules interconnecting the adjoining ordered regions. The tie chains were assumed to vary in length and number according to some type of statistical distribution. The detail structure of the ordered or "crystalline-block" region was not specified but it was assumed to act 
elastically as a unit. Failure of the fiber structure was assumed to to occur mainly by covalent bond rupture. The failure criterion to predict polymer chain scission was based on a Tobolsky-Eyring type rate process theory incorporating a stress-aided activation energy.

\subsection{LIMITATIONS OF. THE STUDY}

1. The study was limited to a very narrow and yet important class of materials; the highly oriented semi-cyrstalline polymer fibers, specifically nylon 6 and nylon 66 , where failure is suspected to be dominated by polymer chain scission.

2. The mathematical model is an idealized model of the polymer fiber microstructure, representing mainly how the structure acts during the failure process and undoubtedly neglects many morphological intricacies.

3. Viscoelastic time effects and entropic forces were not included in the mathematic model, however, portions of the curves attributed to this latter effect have been treated with some success by other authors $(60)$.

4. Uniaxial tensile loadings were the only modes used in testing the fibers. Loading rates were relatively high and time-tofailure short (less than ten minutes) in order to minimize viscoelastic effects and free radical decay.

5. Tests were conducted over a temperature range of $-25^{\circ} \mathrm{C}$ to $+100^{\circ} \mathrm{C}$, although only preliminary testing was conducted above $+50^{\circ} \mathrm{C}$.

\subsection{MAJOR RESULTS}

The most important result from the study was the overall success of 
the mathematical model and failure criterion in predicting experimental behavior for nylon 6 fibers under all attempted loading conditions and at several temperatures. The comparison between predicted and experimental results in most cases was better than qualitative and analysis of the data gave much insight into the fracture process and the fiber microstructure behavior. Specifically the major results are:

1. Reaction rate theory with a stress aided activation energy is a successful failure criterion for predicting covalent bond rupture under mechanically induced stress, i.e.

$$
\frac{d C_{B}}{d t}=K C u
$$

where $k=w_{0} \exp -\frac{\left(U_{0}-\gamma \sigma\right)}{k T}$

and $\frac{d C_{B}}{d t}=$ the rate of polymer chain scission

$C_{u}=$ the number of unbroken polymer chains

$k=$ Boltzmann's constant

$T=$ absolute temperature

$\sigma=$ stress on the polymer chain

with kinetic rate constants

$w_{0}=$ collision parameter

$U_{0}=$ activation energy

$\gamma=$ activation volume

The key to the success of the theory lies in the proper interpretation and application in the stress 0 . The stress, c, is the stress on an individual bond and must be properly related to the macroscopic applied loadings by the micro-macro structure relationships. For the 
particular high-strength fibers investigated by the author, the stress on the individual molecular bonds in the polymer varied greatiy and was determined by two main factors; first, the ratio of the number of polymer chains in the "crystallineblock" region to the number of tie chains in the "critical flaw" regions; and second, the effective statistical distribution of the length of these tie molecules.

2. The characterization and application of this effective distribution is considered by the author to be the most important contribution of this study and the key to its success.

The mathematical model provided direct verification of the need for a distribution in length of tie molecules to explain experimentally observed strength and other fracture related properties of these nylon fibers. EPR provided a method of experimentally characterizing the "apparent or effective" distribution and observing changes or differences in the distribution due to temperature, annealing treatments or manufacturing process. This apparent distribution was characterized from experimental EPR step strain data and found to approximate a Gaussian or normal distribution criterion. The distribution is called an "apparent or effective" distribution due to the ability of tie molecules to assume different effective lengths depending on the local environment. If the effective distribution is narrow (many chains of the same length), then the stress is more uniformly distributed among the tie molecules and the fiber exhibits greater strength. As the effective 
lengths of the tie chains become less uniform, the distribution becomes broader resulting in high stress concentrations in the flaw region and lower overall strength. The relationship between temperature and effective distribution was found to be the key to successful application of the rate process theory. In the microstructure, two basic processes take place in a thermal environment. One might be considered an intramolecular effect and the other an intermolecular effect. The intra-molecular effect is the "strengthening" or "weakening" of the atomic bond in a single chain due to the decrease or increase in thermal vibration of the atoms as a result of temperature changes. The inter-molecular effect is the tightening or loosening of the microstructure inter-atomic forces due to the thermal environment. The degree of this tightening or loosening determines the degree of slippage, twisting, uncoiling, unkinking, etc. that can occur in the local environment. This in turn determines the "effective" end to end chain length of tie molecules in the flaw region and likewise the stress levels of the individual polymer chains. If this distribution in effective chain lengths is broad, high stress levels will exist in the region and effectively weaken the whole structure. Results from this study indicate that unless both intermolecular and intramolecular effects are included in the model, successful correlation with experimental tests at different temperatures would be impossible. 
3. In addition to the effect of the apparent distribution on the strength and other failure characteristics of these fibers, other important microstructure concepts verified by the mathematical model were: (a) chain scission is the main mode of failure; (b) chain scission occurs almost exclusively in "critical flaw" or "weaklink" regions distributed throughout the fiber; and $(c)$, failure properties and fiber strength at a given temperature depend on two main microstructure properties, one, the existance of "weak-link" or "critical flaw" regions that contain a significantly smaller number of load bearing tie molecules than ajoining regions and two, the existance of an effective distribution in length of these tie molecules.

\subsection{SUMMARY OF IMPORTANT DETAILS}

1. Failure in these highly-ordered nylon fibers follows three basic steps, fracture initiation in flaw regions distributed throughout the microstructure, slow "crack" growth due to chain scission in these critical flaw regions, and rapid crack growth due to one or a few of these regions becoming more critical than the others causing catastrophic failure of the sample. The model represents the first two of these processes and can be used successfully to estimate the catastrophic failure point.

2. The mathematical model was constructed using only ratio values to relate microstructure properties to the macroscopic applied loads. Basic input parameters were:

$$
S=\text { the standard deviation of the distribution of tie }
$$


chains in the flaw regions

$\mathrm{RC}=$ the ratio of the number of polymer chains in the "cryblk" region to the number of tie chains in the flaw region

$R L=$ the ratio of the original length of the "cry-blk" region to the original length of the flaw region

$E_{b}=$ the modulus of elasticity of a single polymer chain

$w_{0}=$ the collision parameter representing the thermal vibration frequency of the atom

$U_{0}=$ the activation energy representing the height of the activation barrier which must be overcome for bond breakage to occur.

$\gamma=$ the activation volume representing the area of a chemical bond times the distance necessary to separate the bond to cause rupture.

$T=$ the absolute temperature

$\sigma_{T}$ or $\varepsilon_{T}=$ the applied macroscopic stress (or strain) as a function of time

In addition, an arbitrary parameter $W$ representing the tota 1 chains $/ \mathrm{cm}^{2}$ in the flaw region was included so that predicted chain scission results could be more easily compared with experimental data. As most of the input parameters were relatively unknown, the model itself was used to determine some of these values. The parameters RC, RL, $U_{0}$ and $\gamma$ were determined by comparison of predicted results with four experimental curves (two constant strain rate tests and two constant stress or creep tests). The distribution parameter 
$S$ was determined from characterizing experimental EPR step strain data with a Gaussian distribution standard deviation value. The parameters $E_{b}$ and $w_{0}$ were selected from theoretical considerations. A summary of "best fit" values, their acceptable theoretical range as determined by the model, for nylon $6 \# 1$ at room temperature, and the potential range from previously known data was summarized in Table 4 and is repeated here:

Table 4

Model Parameters

\begin{tabular}{l|c|c|c}
\hline Parameters & "Best Fit" Value & Theoretical Model Range & Potential Range \\
\hline$S$ & 1.25 & 1.25 & no available data \\
RC & 30 & $20-40$ & $3-50$ \\
$R L$ & 5 & $4-10$ & $2-100$ \\
$E_{b}\left(1 \mathrm{~b} / \mathrm{in}^{2}\right)$ & $3 \times 10^{7}$ & $3 \times 10^{7}$ & $2.84 \times 10^{7}$ \\
$\mathrm{~W}_{0}\left(\mathrm{sec}^{-1}\right)$ & $10^{13}$ & $10^{13}$ & $10^{12}-10^{13}$ \\
$U_{0}\left(\mathrm{Kcal}_{\mathrm{mole}}\right)$ & 67.5 & $65-75$ & $43-82$ \\
$\left.\mathrm{O}^{3}\right)$ & 5 & $3-15$ & $1-300$ \\
$W^{3 *}$ & $1 \times 10^{13}$ & $0.5-2.5 \times 10^{13}$ & $1 \times 10^{11}-5 \times 10^{14}$ \\
\hline
\end{tabular}

These results were extremely interesting in that the model parameters not only fell within the range of potential values but provided the best refinement and insight into the application of rate process theory and microstructure faiture properties available to date. Values of the kinetic rate parameters were especially noteworthy as they more closely approximate 
theoretical values than any previously reported from experimental data.

3. Once the "best fit" parameters were determined for nylon 6 \#1 at room temperature, additional theoretical predictions of stress, strain, and chain scission for various loading conditions, i.e. stress and strain rate, creep, low cycle fatigue at several frequencies, stress relaxation and step strain tests, were compared with experimental data. The correlation was very successful and exciting in that parameters determined from very simple tests, i.e. constant load and constant strain rate, could be used to predict macroscopic failure even in low cycle stress fatigue tests. A1though some differences existed between the theoretical predictions and the experimental data, they were reasonably explained by noting that entropic forces and viscoelastic effects were not included in the model. Further refinement of the model could be made by including viscoelastic effects, although these corrections most likely are small based on the good agreement obtained by neglecting them.

4. Comparison of experimental and theoretical constant strain (stress relaxation) data seemed to indicate that the stress relaxation effects observed above six per cent strain in these highiy-oriented fibers could almost wholly be attributed to chain scission.

5. The model predicted bond rupture results in terms of spins/ $\mathrm{cm}^{2}$ while the experimental EPR bond rupture data was in spins/ $\mathrm{cm}^{3}$. Correlation of these results with microstructure 
observations by other techniques led to the conclusion that the "crystalline-block" regions may in fact contain some amorphous or disordered tie molecule regions but that in the fracture process only the weakest ("critical flaw") of these regions contribute significantly to the chain scission process and the remaining ordered and disordered regions comprise a "crystalline-block" region that acts elastically like a unit.

6. Extension of the "best fit" parameters to successful correlation with other temperatures and another type of nylon 6 , i.e. nylon $6 \# 2$, was found to depend on the characterization of the effective distribution for the specific material at the chosen temperature. This characterization was determined from experimental EPR step strain data. Important observations from these test results were:

a. The two nylon 6 samples, i.e. nylon $6 \# 1$ and nylon 6 \#2 were similar in structural parameters except for their "as received" tie chain distribution in the critical flaw regions.

b. The effective distribution is dependent on several factors including the thermal environment, strain rate, plastizers, hydrostatic pressure or other similar items. Permanent changes in the effective distribution can be created by slack or tension annealing treatments and are not to be confused with the essentially reversible effects that occur below approximately $+100^{\circ} \mathrm{C}$. As the temperature was varied the strength of the fiber and the time to failure changed 
significantly. These effects were related to a two fold mechanism occurring in the microstructure due to the thermal environment. These mechanisms have been explained as an intra-molecular and inter-molecular effect where the inter-molecular effect results in the changes in the effective distribution. Experimental results indicate that this change in effective distribution with temperature occurs about the minimum length point in the distribution. Therefore, the strength and position of the failure curve on the time axis is determined by a combination of both effects.

c. Comparatively small changes in the distribution were found to be associated with rather large changes in the fiber strength. The theoretical model qualitatively predicted the effect of changes in the distribution to the maximum strength of the fiber. Assuming a distribution of zero width (all the chains of equal length), and using the "best fit" parameters a theoretical strength of $340 \mathrm{ksi}$ was predicted as the maximum attainable strength for the particular structural configuration of the nylon 6 samples at room temperature. The maximum strength of nyton $6 \# 1$, exhibiting a relatively narrow distribution, i.e. a onestandard deviation value of only ten per cent of the distribution mean strain value, was $165 \mathrm{ksi}$ at room tempature. At any given temperature, an additional important factor in the strength of the fiber was the chain number ratio RC. These two parameters, chain number ratio and 
distribution were the most important factors in determining the strength of the polymer fiber, at a given temperature, after the kinetic rate parameter constants $w_{0}, U_{0}$, and $\gamma$ were established. If the above value of $340 \mathrm{ksi}$ for a zero width distribution was mulitplied by the chain number ratio stress concentration factor, $\mathrm{RC}$, in the above example, a maximum theoretical fiber strength of $10,200 \mathrm{ksi}$ was predicted which is approximately the same as the strength of the single polymer chain.

d. Another observation was that although the total number of tie molecules in the theoretical distribution remained a constant, the amount of chain scission predicted at the maximum stress point and fracture point decreased as the temperature decreased and this number was only a fraction of the tota1. The same observations of a decreasse in chain scissions with temperature at the maximum stress point was experimentally observed. Analyzing these results, the author concluded two things. First, the accumulative number of chain scission recorded by EPR techniques is estimated to be between 45 to 70 per cent of the toal number of tie chains in the "critical flaw" regions and depends on several factors such as temperature, distribution, plasticizers, strain rate, etc. Second, the decrease or increase in the number of chain scissions at the maximum stress point or fracture point did not necessarily mean an increase or decrease in the total number of tie molecules present in the region. In fact, in the temperature 
range from $-25^{\circ} \mathrm{C}$ to $+50^{\circ} \mathrm{C}$ the total number of effective tie molecules in the test samples was considered to remain constant. However, in tests above $+50^{\circ} \mathrm{C}$, chain pul1 out or "unzipping" of the structure may change the effective number of total tie molecules and this then becomes an important third factor in predicting strength and failure behavior.

The importance of the above observations is that researchers should be careful in comparing and analyzing data from EPR chain scission observations.

7. Preliminary studies were conducted on nylon 66 to determine the feasibility of extending the model and theory to other materials. The results were very successful and indicate further work should be pursued. Also, preliminary tests were conducted on a slack annealed nylon 6 fiber and indicated that additional insight into microstructure occurances could be obtained by future additional research.

\subsection{APPLICATION}

The most important application of the results of the reaserch presented is the insight the theory provides to the microstructure behavior during fracture. The approach indicates specific ways that the microstructure can be altered to vary the strength and other important properties of the fiber. With this understanding, special annealing treatments or other processing procedures can be characterized and analyzed by the mathematical model.

Another important application is that of predicting fallure in highstrength fibers. Once the distribution is characterized in the desired 
temperature range and the model parameters are determined from simple strain rate and creep tests the failure properties of the material can be predicted for many different uniaxial loading conditions and temperatures including repeated loadings.

As a final note, the author realizes that the mathematical formulation is not without problems and much further research needs to be conducted to improve and refine the model especially at other temperatures, loading conditions, and on different or modified materials. Bond scission is considered to be the only mote of failure. In reality, there are two competing mechanisms: primary bond rupture and yielding or slip due to secondary bond rupture, viscoelastic effects, etc. Such effects would also be expected to be aided by thermal fluctuations and are only partially accounted for in the model by the apparent broadening in the tie chain distribution. Further corrections can probably be made by assuming stress relaxation in the stress although these corrections may be fairly small based on the good agreement obtained from the model neglecting them. Considering the overal1 complexity of formulating a micro-macro relationship that truly represents the complex polymer structure the results of this study are very gratifying and to the authors knowledge represent the most successful attempt to date to obtain a microscopic failure criterion. Even so, the author is convinced that further refinements to the basic theory will be made by more knowledgeable researchers in the future. 
Page Intentionally Left Blank 
REFERENCES

1. Alexander, W. 0., Scientific American. 217, 254 (1967).

2. "Snythetic Fibers: A Growth Industry." OECD Observer. 12, 10 (1969).

3. Tobolsky, A., and Eyring, H., Journal of Chemical Physics. 11, 125 (1943).

4. Bueche, F., Physical Properties of Polymers. New York: John Wiley and Sons, Inc. 1961.

5. Gubanov, A. I., and Chevychelov, A. D., Soviet Physics--Solid State. 5,62 (1963).

6. Rosen, B., ed., Fracture Processes in Polymer Solids. New York: Interscience Publishers. 1964.

7. von Schmeling, H. H. Kausch-Blecken, Moghe, S. R., and Hsiao, C. C., Journal of Applied Physics. 38, 201 (1967).

8. Zhurkov, S. N., Savostin, A. Y., and Tomashevskii, E. E., Soviet Physics--Doklady. 9 , 968 (1964).

9. Roylance, D. K., DeVries, K. L., and Williams, M. L., Fracture. P. Pratt, ed. London: Chapman and Ha1l. 1969.

10. McCrum, N. G., Read, B. E., and Williams, G., Anelastic and Dielastic Effects in Polymeric Solids. New York: John Wiley and Sons, Inc. 1967.

11. DeVries, K. L., Roylance, D. K., and Williams, M. L., Journal of Polymer Science. Al:8, 237 (1970).

12. Hearle, J. W. S., and Peters, R. H., Fiber Structure. Manchester: The Textile Institute. 1963.

13. Peterlin. A., Journal of Polymer Science. A2:7, 1151 (1969).

14. Bonart, R., and Hosemann, R., Zeitschrift fur Elektrochemie. 64, 314 (1960).

15. Hearle, J. W. S., Journal of Polymer Science. C20, 215 (1967).

16. Boseley, D. E., Journal of Polymer Science. C20, 77 (1967).

17. Keller, A., Philosophical Magazine. 2, 1171 (1957).

18. Fischer, E. W., Goddar, H., and Schmidt, G. F., Macromolecular Chemistry. 117, 170 (1968). 
19. Dismore, P. F., and Statton, W. O., Journal of Polymer Science. B2, 1113 (1964) and $\underline{\mathrm{C} 13}, 133(1966)$.

20. Gubanov, A. I., and Chevychelov, A. D., Soviet Physics--Solid State. 4,4 (1962).

21. Takayanagi, M., Imada, K., and Kajiyama, T., Journal of Polymer Science. C15, 263 (1966).

22. Keith, H. D., Journal of Polymer Science. C20, 117 (1967).

23. Park, J. B., "Fracture Morphology of Nylon 6 Fibers." Unpublished Ph.D. dissertation, Division of Materials Science and Engineering. Department of Mechanical Engineering, University of Utah, Salt Lake City, Utah. 1971.

24. Griffith, A. A., Proceedings of the First International Congress of Applied Mechanics. 55 (1924).

25. Smith, T. L., Journal of Polymer Science. 32, 99 (1958).

26. Rivilin, R. S., and Thomas, A. G., Journal of Polymer Science. 10, 291 (1953).

27. Williams, M. L., International Journal of Fracture Mechanics. I, 29 (1965).

28. von Schmelling, H. H. Kausch-Blecken, Kolloid Zeitschrift and Zeitschrift fur Polymere. 236. 48 (1970).

29. Zhurkov, S. N. and Tomashevskii, E. E., Proceedings of the Conference on the Physical Basis of Yield and Fracture. Oxford, England: Oxford University Press. 1966.

30. Roylance, D. K., "An EPR Investigation of Polymer Fracture." An unpublished Ph.D. dissertation, Department of Mechanical Engineering, University of Utah, Salt Lake City, Utah. 1968.

31. DeVries, K. L., Roylance, D. K., and Williams, M. L., International Journal of Fracture Mechanics. 7, 197 (1971).

32. DeVries, K. L., and Farris, R. J., International Journal of Fracture Mechanics. 6, 411 (1970).

33. Verma, G. S. P., and Peterlin, A., Polymer Preprints. 10, 1051. (1969).

34. von Schmeling, H. H. Kausch-Blecken, and Becht, J., Journal of Rheological Acta. 2, 137 (1970).

35. von Schmeling, H. H. Kausch-Blecken, International Journal of Fracture Mechanics. $\underline{6}, 301$ (1970).

36. von Schmeling, H. H. Kausch-Blecken, Journal of Macromolecular Science--Reviews of Macromolecular Chemistry. C4(2), 243 (1970). 
37. Chevychelov, A.D., Polymer Mechanics. 3, 5 (1970).

38. DeVries, K. L., Lloyd, B. A., and Williams, M. L., Journal of Applied Physics. 42, 4644 (1971).

39. Bershohn, M. and Baird, J. C., Electron Paramagnetic Resonance. New York: W. A. Benjamin, Inc. 1966.

40. Carrington, A. and McLachlan, A. D., Introduction to Magnetic Resonance with Applications to Chemistry and Chemical Physics. New York: Harper and Row. 1967.

41. E-3 EPR Spectrometer System Publication No. 87-118-001. Palo Alto, California: Varian Associates. 1966.

42. Becht J. and Fischer, H., Kolloid-Zeitschrift. 229, 1967 (1969).

43. Backman, D. K., "An EPR Investigation of Polymer Fracture Surfaces." Unpublished Ph.D. dissertation, Department of Mechanical Engineering, University of Utah, Salt Lake City, Utah. 1969.

44. Becht, J., DeVries, K. L., and von Schmelling, H. H. Kausch-Blecken, European Polymer Journal. ㄱ, 105 (1971).

45. Backman, D. K. and DeVries, K. L., Journal of Polymer Science. Al:7, 2125 (1969).

46. Predecki, P. and Statton, W. 0., Journal of Applied Physics. 37, 4053 (1966).

47. Perepelkin, K. E., Polymer Mechanics. 2, 536 (1969).

48. Holmes, D. R., Bunn, C. W., and Smith, D. J., Journal of Polymer Science. 17, 159 (1955).

49. Geil, P. H., Polymer Single Crystals. New York: Interscience Pub1ishers. 1963.

50. Statton, W. 0., Journal of Polymer Science. C18, 33 (1967).

51. Meinel, G. and Peterlin, A., Journal of Polymer Science. A2:6, 587 (1968).

52. Hess, K. and Kiessig, H., Zeitschrift fur Physikalische Chemie. 193, $196(1944)$.

53. Statton, W. O., Journal of Polymer Science. 41, 143 (1959).

54. Campbel1, D. and Peterlin, A., Polymer Letters. 6, 481 (1968). 
55. Treloar, L. R. G., Polymer. 1, 95 (1960).

56. Roberts, J. D. and Caserio, M. C., Basic Principles of Organic Chemistry. New York: W. A. Benjamin, Inc. 1965.

57. Gerö, L., Journal of Chemical Physics. · 16, 1011 (1948).

58. Zakrevskii, V. A., Tomashevskii, E. E.; a.nd Baptizmanskii, V. V., Soviet Physics--Solid State. 9, 1118 (1967).

59. Zhurkov, S. N., International Journal of Fracture Mechanics. 1 , $311(1965)$.

60. Pechhold, W., Journal of Polymer Science. C32, 123 (1971).

61. Peterlin, A., Personal communication.

62. Zhurkov, S. N., Kuksenko, V. S., and Slutsker, A. I., Fracture.

P. Pratt, ed. London: Chapman and Hall. 1969. 
Page Intentionally Left Blank 
VITA

NAME:

BIRTHPLACE :

BIRTHDATE :

EDUCATION:

DEGREES:

AWARDS AND HONORS:

PROFESSIONAL SOCIETIES AND CERTIFICATES:

EXPERIENCE:
Ben Ashton Lloyd

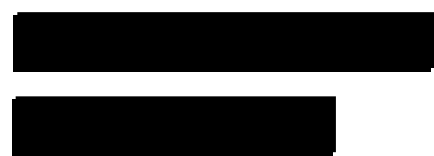

Lafayette Elementary School

Horace Mann Junior High School West High School

Arizona State University University of Utah

BSME, University of Utah 1963

Ph. D. University of Utah 1972

Athletic and Academic Scholarships awarded during undergraduate studies

University of Utah Wrestling Team Scholarship Trophy 1958

3rd Place Mountain States Conference Wrestling Championship 137 Ib Division 1958

2nd Place Intermountain Association AAU Wrestling Championship 137 lb Senior Division 1958

Honarary Fraternities

Phi Kappa Phi

Tau Beta Pi

Pi Tau Sigma

Phi Eta Sigma

NDEA Fellowship 1970-1971

First Place Award, Unlimited Division, 21st Annual AIAA, Western Regional Student Paper Competition 1971

Engineer in Training Certificate 1963 American Institute of Aeronautics and Astronautics

American Society of Mechanical Engineers

International Association for Dental Research

Junior Engineer, Sperry Utah Company 1963-64

Project Engineer, Sperry Phoenix Company 1964-66

Senior Project Engineer, Univac, Salt Lake City, 1966-68

Research Assistant, University of Utah 1968-71

Research Associate, University of Utah 1971-72 
PUBLICATIONS:

Sutton, B., Gardner, C. N., Lloyd, B. A., "Overall Evaluation and Mobility Prediction of Ground Support Vehicles for Weapons Systems." Society of Automotive Engineers, Inc. 39, Dec. 1964.

DeVries, K. L., Lloyd, B. A., Williams, M. L., "A Reaction Rate Model for Fracture in Polymeric Fibers." Journal of Applied Physics. 42, 4644,1971

McGinley, M. B., Lloyd, B. A., Despain, R. R., and Brown, W. S., "Tensile Strength of Ename1." Abstract No. 871, International Association for Dental Research 50th Annual Conference. March 1972.

Lloyd, B. A., DeVries, K. L., Williams, M. L., "Fracture Behavior in Nylon 6 Fibers." accepted for publication in the Journal of Polymer Science. 1972. 